\title{
NICA-TOEPLITZ ALGEBRAS ASSOCIATED WITH PRODUCT SYSTEMS OVER RIGHT LCM SEMIGROUPS
}

\author{
BARTOSZ K. KWAŚNIEWSKI AND NADIA S. LARSEN
}

\begin{abstract}
We prove uniqueness of representations of Nica-Toeplitz algebras associated to product systems of $C^{*}$-correspondences over right LCM semigroups by applying our previous abstract uniqueness results developed for $C^{*}$-precategories. Our results provide an interpretation of conditions identified in work of Fowler and Fowler-Raeburn, and apply also to their crossed product twisted by a product system, in the new context of right LCM semigroups, as well as to a new, Doplicher-Roberts type $C^{*}$-algebra associated to the Nica-Toeplitz algebra. As a derived construction we develop Nica-Toeplitz crossed products by actions with completely positive maps. This provides a unified framework for Nica-Toeplitz semigroup crossed products by endomorphisms and by transfer operators. We illustrate these two classes of examples with semigroup $C^{*}$-algebras of right and left semidirect products.
\end{abstract}

\section{INTRODUCTION}

Product systems of $C^{*}$-correspondences were introduced by Fowler following ideas of Arveson. As spelled out in [11], Fowler's construction served as motivation for his investigation with Raeburn into uniqueness theorems for $C^{*}$-algebras arising as certain twisted crossed products over positive cones in quasi-lattice ordered groups, [12]. Three uniqueness theorems in this context have dominated the attention: [13, Theorem 2.1], [11, Theorem 7.2] and [12, Theorem 5.1]. All three give a necessary condition for faithfulness of a representation $\pi$ of a Toeplitztype $C^{*}$-algebra $\mathcal{T}_{X}$ or $\mathcal{N} \mathcal{T}(X)$, where $X$ is generic notation for a single $C^{*}$-correspondence or a product system of such over a semigroup, and $\pi$ arises from a representation of $X$.

Two aspects are striking here: the first is that the necessary condition, to which we choose to refer as condition $(C)$ - for compression or for Coburn, who proved the archetypical result of this form - is only sufficient when the left action in each correspondence is by generalized compacts. The second is that, in the aforementioned results on product systems, an auxiliary $C^{*}$-algebra is involved. It has the structural appearance of a crossed product twisted by a product system and a somewhat unaccountable involvement in the uniqueness of representations of $\mathcal{N} \mathcal{T}(X)$.

The first main point we make in the present paper is that there is another $C^{*}$-algebra for which uniqueness of representations coming from $X$ is precisely encoded, as a necessary and sufficient condition, by condition (C). This $C^{*}$-algebra, which we generically denote $\mathcal{D} \mathcal{R}(\mathcal{N} \mathcal{T}(X))$, bears the flavor of a Doplicher-Roberts algebra for $\mathcal{N} \mathcal{T}(X)$. The second point we make is that uniqueness of a representation $\pi$ of $\mathcal{N} \mathcal{T}(X)$ can, in good situations, be precisely encoded by a weaker condition than (C) which we call Toeplitz covariance. The third point we make is that the strategy for proving these results relies on our previous work on $C^{*}$-precategories developed in [19], and as a very satisfactory bonus provides a clear picture of how $\mathcal{N} \mathcal{T}(X)$, the Fowler-Raeburn crossed product twisted by a product system and $\mathcal{D} \mathcal{R}(\mathcal{N} \mathcal{T}(X))$ are included in each other, respectively, and how uniqueness of representations on $\mathcal{D} \mathcal{R}(\mathcal{N} \mathcal{T}(X))$ sieves down to corresponding results on the smaller subalgebras. Together,

Date: 15 June 2017. Revised 17 November 2017 and 18 September 2018. 
these three uniqueness results offer a different picture of endeavors by many hands over several decades. In addition we extend these results beyond the scope of quasi-lattice ordered pairs, which is non-trivial as LCM semigroups allow invertible elements and might not be cancellative, cf. [19].

As an application of our uniqueness results we define a Nica-Toeplitz crossed product for a dynamical system involving a semigroup action of completely positive maps on a $C^{*}$ algebra. For a single completely positive map, a similar construction was proposed by the first named author in [18]. In this new setup of semigroup actions by completely positive maps our construction models Toeplitz-type crossed products in two important contexts: actions by endomorphisms, see e.g. [11] (where the assumptions on the acting semigroup and the conventions on covariance are different), and actions by transfer operators, see e.g. [25] where the acting semigroup is abelian. We formulate uniqueness theorems for our crossed products, and illustrate the two classes of actions with semigroup $C^{*}$-algebras as in $\mathrm{Li}$ [27], through the perspective of algebraic dynamical systems developed by the second named author in collaboration with Brownlowe and Stammeier [2]. The left-semidirect product semigroup $C^{*}$ algebras coming from [2] will serve to motivate crossed products by transfer operators, and, somehow unexpectedly though in hindsight not that surprisingly, right semidirect product semigroup $C^{*}$-algebras will motivate crossed products by endomorphisms.

The paper is organized as follows. In a preliminaries section we review briefly the basics of $C^{*}$-correspondences and product systems of these, after which we collect the main ingredients needed about $C^{*}$-precategories and their $C^{*}$-algebras from [19]. In section 2.1 we associate a $C^{*}$-precategory to a product system $X$ of $C^{*}$-correspondences over a right LCM semigroup $P$ and in section 2.2 we use it to construct a Doplicher-Roberts version $\mathcal{D} \mathcal{R}(\mathcal{N} \mathcal{T}(X))$ and a reduced version of $\mathcal{N} \mathcal{T}(X)$. We introduce conditions under which representations of $X$ give rise to faithful representations of the core subalgebras of $\mathcal{N} \mathcal{T}(X)$ and $\mathcal{D} \mathcal{R}(\mathcal{N} \mathcal{T}(X))$. In subsection 2.3 we prove uniqueness results for $\mathcal{N} \mathcal{T}(X)$ and $\mathcal{D} \mathcal{R}(\mathcal{N} \mathcal{T}(X))$ and discuss some implications. In subsection 2.4 we extend this discussion by introducing $C^{*}$-algebras $\mathcal{F} \mathcal{R}(X)$ generalizing semigroup $C^{*}$-algebras twisted by product systems studied by Fowler and Raeburn, see [12], [11]. In section 3 we introduce Nica-Toeplitz crossed products of a $C^{*}$-algebra by the action of a right LCM semigroup of completely positive maps, and prove uniqueness results for the two major types of examples, crossed products by endomorphisms and by transfer operators. Finally, in section 4 we show that the Nica-Toeplitz crossed products by endomorphisms and by transfer operators can be perfectly embodied by semigroup $C^{*}$-algebras associated to right and left semidirect products of semigroups, respectively. By specializing the uniqueness results to these contexts we generalize and complement earlier results from [23] and [2].

While we were laying the last hand on this part II, another paper appeared [10] in which Fletcher takes on clarifying the uniqueness result [11, Theorem 7.2] for $\mathcal{N} \mathcal{T}(X)$ in the context of quasi-lattice ordered pairs.

0.1. Acknowledgements. The research leading to these results has received funding from the European Union's Seventh Framework Programme (FP7/2007-2013) under grant agreement number 621724. B.K. was partially supported by the NCN (National Centre of Science) grant number 2014/14/E/ST1/00525. Part of the work was carried out when B.K. participated in the Simons Semester at IMPAN - Fundation grant 346300 and the Polish Government MNiSW 2015-2019 matching fund, the participation of both authors in the program "Classification of operator algebras: complexity, rigidity, and dynamics" at the Mittag-Leffler Institute (Sweden) in January 2016. 


\section{Preliminaries}

1.1. LCM semigroups. We refer to [3] and [1] and the references therein for basic facts about right LCM semigroups. All semigroups considered in this paper will have an identity $e$. We let $P^{*}$ be the group of units, or invertible elements, in $P$. A principal right ideal in $P$ is a right ideal in $P$ of the form $p P=\{p s: s \in P\}$ for some $p \in P$. The relation of inclusion on the principal right ideals induces a left invariant preorder on $P$ given by $p \leq q$ when $q P \subseteq p P$. Clearly $\leq$ is a partial order if and only if $P^{*}=\{e\}$.

A semigroup $P$ is a right $L C M$ semigroup if the family $\{p P\}_{p \in P}$ of principal right ideals extended by the empty set is closed under intersections, that is if for every pair of elements $p, q \in P$ we have $p P \cap q P=\emptyset$ or $p P \cap q P=r P$ for some $r \in P$. In the case that $p P \cap q P=r P$, the element $r$ is a right least common multiple ( $L C M$ ) of $p$ and $q$. If $P$ is a right LCM semigroup then we refer to $J(P):=\{p P\}_{p \in P} \cup\{\emptyset\}$ as the semilattice of principal right ideals of $P$. Right LCMs in a right LCM semigroup are determined up to multiplication from the right by an invertible element. Namely, if $p P \cap q P=r P$, then $p P \cap q P=t P$ if and only if there is $h \in P^{*}$ such that $t=r h$.

Example 1.1. (a) One of the most known and studied examples of right LCM semigroups are positive cones in quasi-lattice ordered groups, introduced by Nica [29]. In fact, $P$ is a positive cone in a weakly quasi-lattice ordered group $(G, P)$ if and only if $P$ is an LCM subsemigroup of a group $G$ such that $P^{*}=\{e\}$.

(b) In semigroup theory, notions similar to right LCM have been known for some time, see e.g. [26]. New large classes of right LCM semigroups with relevance to $C^{*}$-algebraic context were identified in [3]. Semidirect product semigroups which are right LCM semigroups were studied in $[1,2]$. More on this in section 4 .

We recall from [19, Definition 2.4] that a controlled map of right LCM semigroups is an identity preserving homomorphism $\theta: P \rightarrow \mathcal{P}$ between right LCM semigroups $P, \mathcal{P}$ such that $\theta\left(P^{*}\right)=\mathcal{P}^{*}$ and for all $s, t \in P$ with $s P \cap t P=r P$ we have $\theta(s) \mathcal{P} \cap \theta(t) \mathcal{P}=\theta(r) \mathcal{P}$ and $\theta(s)=\theta(t)$ only if $s=t$.

Example 1.2. Let $P_{i}, i \in I$, be a family of right LCM semigroups. Put $P:=\prod_{i \in I}^{*} P_{i}$ and $\mathcal{P}:=\bigoplus_{i \in I} P_{i}$. The homomorphism $\theta: P \rightarrow \mathcal{P}$ which is the identity on each $P_{i}, i \in I$, is a controlled map of right LCM semigroups, by [19, Proposition 2.3].

1.2. $C^{*}$-correspondences and product systems. The notion of a $C^{*}$-correspondence $X$ over a $C^{*}$-algebra $A$ and its associated Toeplitz algebra $\mathcal{T}(X)$ are standard, and we refer to $[32,16,13]$ for details. We recall from [11] that a product system over a semigroup $P$ with coefficients in a $C^{*}$-algebra $A$ is a semigroup $X=\bigsqcup_{p \in P} X_{p}$, with each $X_{p}$ a $C^{*}$-correspondence over $A$, equipped with a semigroup homomorphism $d: X \rightarrow P$ such that $X_{p}=d^{-1}(p)$ is a $C^{*}$-correspondence over $A$ for each $p \in P, X_{e}$ is the standard bimodule ${ }_{A} A_{A}$, and the multiplication on $X$ extends to isomorphisms $X_{p} \otimes_{A} X_{q} \cong X_{p q}$ for $p, q \in P \backslash\{e\}$ and coincides with the right and left actions of $X_{e}=A$ on each $X_{p}$. For each $p \in P$ we write $\langle\cdot, \cdot\rangle$ for the $A$-valued inner product on $X_{p}$ and we denote $\phi_{p}$ the homomorphism from $A$ into $\mathcal{L}\left(X_{p}\right)$ which implements the left action of $A$ on $X_{p}$.

A Hilbert A-bimodule is a $C^{*}$-correspondence which is also a left Hilbert module such that ${ }_{A}\langle x, y\rangle \cdot z=x \cdot\langle y, z\rangle_{A}$ for all $x, y, z \in X$. An equivalence $A$-bimodule is a Hilbert bimodule which is full over left and right. We say that two Hilbert $A$-bimodules $X, Y$ are Morita equivalent if there is an equivalence $A$-bimodule $E$ such that $X \otimes_{A} E \cong Y \otimes_{A} E$.

Remark 1.3. A product system $X$ is (left) essential if each $C^{*}$-correspondence $X_{p}, p \in P$, is essential. We claim that $X$ is automatically essential whenever the group $P^{*}$ of units in $P$ 
is non-trivial. Indeed, for any $h \in P^{*} \backslash\{e\}$ and $p \in P$ we have natural isomorphisms

$$
X_{p}=X_{h h^{-1} p} \cong X_{h} \otimes_{A} X_{h^{-1}} \otimes_{A} X_{p} \cong X_{e} \otimes_{A} X_{p} \cong \phi_{p}(A) X_{p}
$$

that give $X_{p}=\phi_{p}(A) X_{p}$. Moreover, isomorphisms $X_{h} \otimes_{A} X_{h^{-1}} \cong A_{A}$ and $X_{h^{-1}} \otimes_{A} X_{h} \cong A_{A}$ imply that $X_{h}$ and $X_{h^{-1}}$ are mutually adjoint Hilbert bimodules, i.e. there is an antilinear isometric bijection $b_{h}: X_{h} \rightarrow X_{h^{-1}}$ such that $b_{h}(a b)=b_{h}(b) a$ and $b_{h}(b a)=a b_{h}(b)$ for all $a \in A$ and $b \in X_{h}$, cf. [2, Remark 6.2]. In particular, the family of Banach spaces $\left\{X_{h}\right\}_{h \in P^{*}}$ together with multiplication inherited from $X$ and involution defined by $b^{*}:=b_{h}(b)$, for $b \in X_{h}, h \in P^{*}$, is a saturated Fell bundle over the (discrete) group $P^{*}$, cf. [8].

Given a product system $X$ and $p, q \in P$ with $p \neq e$, there is a homomorphism $\iota_{p}^{p q}: \mathcal{L}\left(X_{p}\right) \rightarrow$ $\mathcal{L}\left(X_{p q}\right)$ characterized by

$$
\iota_{p}^{p q}(S)(x y)=(S x) y \text { for all } x \in X_{p}, y \in X_{q} \text { and } S \in \mathcal{L}\left(X_{p}\right) .
$$

For each $p \in P, \mathcal{K}\left(A, X_{p}\right)$ is a $C^{*}$-correspondence with $A$-valued inner product $\langle T, S\rangle_{A}=$ $T^{*} S$ and pointwise actions. In fact, see [33, Lemma 2.32], there is a $C^{*}$-correspondence isomorphism $X_{p} \cong \mathcal{K}\left(A, X_{p}\right)$ implemented by the map

$$
X_{p} \ni x \mapsto t_{x} \in \mathcal{K}\left(A, X_{p}\right) \quad \text { where } t_{x}(a)=x \cdot a \text {. }
$$

One defines $\iota_{e}^{p}: \mathcal{K}\left(X_{e}\right) \rightarrow \mathcal{L}\left(X_{p}\right)$ by letting $\iota_{e}^{p}\left(t_{a}\right)=\phi_{p}(a)$ for $p \in P, a \in A$, see [34, Section $2.2]$.

A representation of the product system $X$ in a $C^{*}$-algebra $B$ is a semigroup homomorphism $\psi: X \rightarrow B$, where $B$ is viewed as a semigroup with multiplication, such that $\left(\psi_{e}, \psi_{p}\right)$ is a representation of the $C^{*}$-correspondence $X_{p}$, for all $p \in P$, where we put $\psi_{p}:=\left.\psi\right|_{X_{p}}$ for all $p \in P$. The Toeplitz algebra $\mathcal{T}(X)$ is the $C^{*}$-algebra generated by a universal representation of $X$.

In the case of a quasi-lattice ordered pair $(G, P)$, Fowler introduced in [11] the notions of compactly aligned product system over $P$ and Nica covariant representation of it. In [2], these concepts were extended to the case when $P$ is a right LCM semigroup. Given a right LCM semigroup $P$, a product system $X$ over $P$ is called compactly aligned if for all $p, q \in P$ such that there is a right LCM $r$ for $p, q$, then $\iota_{p}^{r}(S) \iota_{q}^{r}(T) \in \mathcal{K}\left(X_{r}\right)$ whenever $S \in \mathcal{K}\left(X_{p}\right)$ and $T \in \mathcal{K}\left(X_{q}\right)$. Assume $X$ is a compactly aligned product system over $P$ and let $\psi$ be a representation of $X$ in a $C^{*}$-algebra. For each $p \in P$, denote $\psi^{(p)}$ the Pimsner $*$-homomorphism defined on $\mathcal{K}\left(X_{p}\right)$ by $\psi^{(p)}\left(\Theta_{x, y}\right)=\psi_{p}(x) \psi_{p}(y)^{*}$ for $x, y \in X_{p}$. Then $\psi$ is Nica covariant if

$$
\psi^{(p)}(S) \psi^{(q)}(T)= \begin{cases}\psi^{(r)}\left(\iota_{p}^{r}(S) \iota_{q}^{r}(T)\right) & \text { if } p P \cap q P=r P \\ 0 & \text { otherwise }\end{cases}
$$

for all $S \in \mathcal{K}\left(X_{p}\right)$ and $T \in \mathcal{K}\left(X_{q}\right)$ (see also [11, Definition 5.7]). The Nica Toeplitz algebra $\mathcal{N} \mathcal{T}(X)$ is the $C^{*}$-algebra generated by a Nica covariant representation $i_{X}$ which is universal in the following sense: if $\psi$ is a Nica covariant Toeplitz representation of $X$ in $B$ there is a *-homomorphism $\psi_{*}: \mathcal{N} \mathcal{T}(X) \rightarrow B$ such that $\psi_{*} \circ i_{X}=\psi$.

1.3. $C^{*}$-precategories. $C^{*}$-precategories should be regarded as non-unital versions of $C^{*}$ categories, cf. [15], [5]. We give here a very brief account, for more details and background material on $C^{*}$-precategories, see [17], [19].

Recall that a $C^{*}$-precategory $\mathcal{L}$ with object set $P$ is identified with a collection of Banach spaces $\{\mathcal{L}(p, q)\}_{p, q \in P}$, viewed as morphisms, equipped with bilinear maps, viewed as composition of morphisms, $\mathcal{L}(p, q) \times \mathcal{L}(q, r) \ni(a, b) \mapsto a b \in \mathcal{L}(p, r), p, q, r \in P$, satisfying $\|a b\| \leq\|a\| \cdot\|b\|$, and an antilinear involutive contravariant mapping ${ }^{*}: \mathcal{L} \rightarrow \mathcal{L}$ such that if $a \in \mathcal{L}(p, q)$, then $a^{*} \in \mathcal{L}(q, p)$ and the $C^{*}$-equality $\left\|a^{*} a\right\|=\|a\|^{2}$ holds. In particular, $\mathcal{L}(p, p)$ 
is naturally a $C^{*}$-algebra, and we require that for every $a \in \mathcal{L}(q, p)$ the element $a^{*} a$ is positive in the $C^{*}$-algebra $\mathcal{L}(p, p)$.

An ideal in a $C^{*}$-precategory $\mathcal{L}$ is a collection $\mathcal{K}=\{\mathcal{K}(p, q)\}_{p, q \in P}$ of closed linear subspaces $\mathcal{K}(p, q)$ of $\mathcal{L}(p, q), p, q \in P$, such that

$$
\mathcal{L}(p, q) \mathcal{K}(q, r) \subseteq \mathcal{K}(p, r) \quad \text { and } \quad \mathcal{K}(p, q) \mathcal{L}(q, r) \subseteq \mathcal{K}(p, r)
$$

for all $p, q, r \in P$. Then $\mathcal{K}$ is automatically selfadjoint and hence a $C^{*}$-precategory. An ideal $\mathcal{K}$ in $\mathcal{L}$ is uniquely determined by the $C^{*}$-algebras $\{\mathcal{K}(p, p)\}_{p \in P}$, which are in fact ideals in the corresponding $C^{*}$-algebras $\{\mathcal{L}(p, p)\}_{p \in P}$. We say that $\mathcal{K}$ is an essential ideal in $\mathcal{L}$ if $\mathcal{K}(p, p)$ is an essential ideal in $\mathcal{L}(p, p)$, for every $p \in P$.

A representation $\Psi: \mathcal{L} \rightarrow B$ of a $C^{*}$-precategory $\mathcal{L}$ in a $C^{*}$-algebra $B$ is a family $\Psi=$ $\left\{\Psi_{p, q}\right\}_{p, q \in P}$ of linear operators $\Psi_{p, q}: \mathcal{L}(p, q) \rightarrow B$ such that

$$
\Psi_{p, q}(a)^{*}=\Psi_{q, p}\left(a^{*}\right), \quad \text { and } \quad \Psi_{p, r}(a b)=\Psi_{p, q}(a) \Psi_{q, r}(b),
$$

for all $a \in \mathcal{L}(p, q), b \in \mathcal{L}(q, r)$. Then automatically all the maps $\Psi_{p, q}, p, q \in P$, are contractions, and they all are isometries if and only if all the maps $\Psi_{p, p}, p \in P$, are injective. In the latter case we say that $\Psi$ is injective. We denote by $C^{*}(\Psi(\mathcal{L}))$ the $C^{*}$-algebra generated by the spaces $\Psi(\mathcal{L}(p, q)), p, q \in P$. A representation $\Psi$ of $\mathcal{L}$ on a Hilbert space $H$ is a representation of $\mathcal{L}$ in the $C^{*}$-algebra $\mathcal{B}(H)$ of all bounded operators on $H$. If in addition $C^{*}(\Psi(\mathcal{L})) H=H$ we say that the representation $\Psi$ is nondegenerate.

If $\mathcal{K}$ is an ideal in a $C^{*}$-precategory $\mathcal{L}$ and $\Psi=\left\{\Psi_{p, q}\right\}_{p, q \in P}$ is a representation of $\mathcal{K}$ on a Hilbert space $H$, then there is a unique extension $\bar{\Psi}=\left\{\bar{\Psi}_{p, q}\right\}_{p, q \in P}$ of $\Psi$ to a representation of $\mathcal{L}$ such that the essential subspace of $\bar{\Psi}_{p, q}$ is contained in the essential subspace of $\Psi_{p, q}$, for every $p, q \in P$. Namely, we have

$$
\bar{\Psi}_{p, q}(a)(\mathcal{K}(q, q) H)^{\perp}=0, \quad \text { and } \quad \bar{\Psi}_{p, q}(a) \Psi_{q, q}(b) h=\Psi_{p, q}(a b) h
$$

for all $a \in \mathcal{L}(p, q), b \in \mathcal{K}(q, q), h \in H$. Moreover, $\bar{\Psi}$ is injective if and only if $\Psi$ is injective and $\mathcal{K}$ is an essential ideal in $\mathcal{L}$.

1.4. Right tensor $C^{*}$-precategories and their $C^{*}$-algebras. We recall the basic definitions and facts from [19, Section 3]. A right-tensor $C^{*}$-precategory is a $C^{*}$-precategory $\mathcal{L}=\{\mathcal{L}(p, q)\}_{p, q \in P}$ whose objects form a semigroup $P$ with identity $e$ and which is equipped with a semigroup $\left\{\otimes 1_{r}\right\}_{r \in P}$ of endomorphisms of $\mathcal{L}$ sending $p$ to $p r$, for all $p, r \in P$, and $\otimes 1_{e}=i d$. More precisely, we have linear operators $\mathcal{L}(p, q) \in a \mapsto a \otimes 1_{r} \in \mathcal{L}(p r, q r)$ such that for each $a \in \mathcal{L}(p, q), b \in \mathcal{L}(q, s)$, and $p, q, r, s \in P$ we have

$$
\left(\left(a \otimes 1_{r}\right) \otimes 1_{s}\right)=a \otimes 1_{r s}, \quad\left(a \otimes 1_{r}\right)^{*}=a^{*} \otimes 1_{r}, \quad\left(a \otimes 1_{r}\right)\left(b \otimes 1_{r}\right)=(a b) \otimes 1_{r} .
$$

We refer to $\left\{\otimes 1_{r}\right\}_{r \in P}$ as to a right tensoring on $\mathcal{L}=\{\mathcal{L}(p, q)\}_{p, q \in P}$.

If $\mathcal{K}$ is an ideal in a right-tensor $C^{*}$-precategory $\mathcal{L}$, we say that $\mathcal{K}$ is $\otimes 1$-invariant, and write $\mathcal{K} \otimes 1 \subseteq \mathcal{K}$, if $\mathcal{K}(p, p) \otimes 1_{r} \subseteq \mathcal{K}(p r, p r)$ for all $p, r \in P$. One can show that $\mathcal{K} \otimes 1 \subseteq \mathcal{K}$ if and only if $\mathcal{K}(p, q) \otimes 1_{r} \subseteq \mathcal{K}(p r, q r)$ for all $p, q, r \in P$. Right tensor representations and the corresponding Toeplitz algebras are defined for all ideals, $\otimes 1$-invariant or not, in some $\mathcal{L}$.

Let $\mathcal{K}$ be an ideal in a right-tensor $C^{*}$-precategory $\mathcal{L}$. We say that a representation $\Psi$ : $\mathcal{K} \rightarrow B$ of $\mathcal{K}$ in a $C^{*}$-algebra $B$ is a right-tensor representation if for all $a \in \mathcal{K}(p, q), b \in \mathcal{K}(s, t)$ such that $s P \subseteq q P$ we have

$$
\Psi(a) \Psi(b)=\Psi\left(\left(a \otimes 1_{q^{-1} s}\right) b\right) .
$$

Note that, since $\mathcal{K}$ is an ideal, the right hand side of (1.4) makes sense. One can show there is an injective right-tensor representation $t_{\mathcal{K}}: \mathcal{K} \rightarrow \mathcal{T}_{\mathcal{L}}(\mathcal{K})$ with the universal property that for every right-tensor representation $\Psi$ of $\mathcal{K}$ there is a homomorphism $\Psi \times P$ of $\mathcal{T}_{\mathcal{L}}(\mathcal{K})$ such 
that $\Psi \times P \circ t_{\mathcal{K}}=\Psi$, and $\mathcal{T}_{\mathcal{L}}(\mathcal{K})=C^{*}\left(t_{\mathcal{K}}(\mathcal{K})\right)$. We call $\mathcal{T}_{\mathcal{L}}(\mathcal{K})$ the Toeplitz algebra of $\mathcal{K}$. We write $\mathcal{T}(\mathcal{L})$ for the Toeplitz algebra $\mathcal{T}_{\mathcal{L}}(\mathcal{L})$ associated to $\mathcal{L}$, viewed as an ideal in itself.

If the underlying semigroup is right LCM, then for well-aligned ideals we can make sense of a condition of Nica type, which is stronger than (1.4).

Let $\left(\mathcal{L},\left\{\otimes 1_{r}\right\}_{r \in P}\right)$ be a right-tensor $C^{*}$-precategory over a right LCM semigroup $P$. An ideal $\mathcal{K}$ in $\mathcal{L}$ is well-aligned in $\mathcal{L}$ if for all $a \in \mathcal{K}(p, p), b \in \mathcal{K}(q, q)$ we have

$$
\left(a \otimes 1_{p^{-1} r}\right)\left(b \otimes 1_{q^{-1} r}\right) \in \mathcal{K}(r, r) \quad \text { whenever } \quad p P \cap q P=r P .
$$

By [19, Lemma 3.7], for any ideal $\mathcal{K}$ condition (1.5) implies the formally stronger condition that for every $a \in \mathcal{K}(p, q), b \in \mathcal{K}(s, t)$ we have

$$
\left(a \otimes 1_{q^{-1} r}\right)\left(b \otimes 1_{s^{-1} r}\right) \in \mathcal{K}\left(p q^{-1} r, t s^{-1} r\right) \quad \text { whenever } \quad q P \cap s P=r P .
$$

Standing assumptions 1.4. For every well-aligned ideal $\mathcal{K}$ in $\mathcal{L}$, in this paper we will also assume the following condition

- $\mathcal{K}$ is $\otimes 1$-nondegenerate, $[19$, Definition 9.6] that is

$$
\left(\mathcal{K}(p, p) \otimes 1_{r}\right) \mathcal{K}(p r, p r)=\mathcal{K}(p r, p r) \text { for every } p \in P \backslash P^{*} \text { and } r \in P .
$$

- $\mathcal{K}$ satisfies condition (7.6) in [19, Proposition 7.6] for $t=e$, that is

$$
\overline{\mathcal{K}(p, e) \mathcal{K}(e, p)} \text { is an essential ideal in the } C^{*} \text {-algebra } \mathcal{L}(p, p) \text {, for every } p \in P \text {. }
$$

These conditions will be satisfied by right-tensor $C^{*}$-precategories arising from product systems.

1.5. Nica-Toeplitz algebras associated with right-tensor $C^{*}$-precategories. Let us fix a right-tensor $C^{*}$-precategory $\left(\mathcal{L},\left\{\otimes 1_{r}\right\}_{r \in P}\right)$ over an LCM semigroup $P$, and a well-aligned ideal $\mathcal{K}$ in $\mathcal{L}$. A representation $\Psi: \mathcal{K} \rightarrow B$ of $\mathcal{K}$ in a $C^{*}$-algebra $B$ is $N i c a$ covariant if for all $a \in \mathcal{K}(p, q), b \in \mathcal{K}(s, t)$ we have

$$
\Psi(a) \Psi(b)= \begin{cases}\Psi\left(\left(a \otimes 1_{q^{-1} r}\right)\left(b \otimes 1_{s^{-1} r}\right)\right) & \text { if } q P \cap s P=r P \text { for some } r \in P, \\ 0 & \text { otherwise. }\end{cases}
$$

Note that by (1.6) the right hand side of (1.9) makes sense. By [19] there is an injective Nica covariant representation $i_{\mathcal{K}}: \mathcal{K} \rightarrow \mathcal{N} \mathcal{T}_{\mathcal{L}}(\mathcal{K})$ with the universal property: for every Nica covariant representation $\Psi$ of $\mathcal{K}$ there is a homomorphism $\Psi \rtimes P$ of $\mathcal{N} \mathcal{T}_{\mathcal{L}}(\mathcal{K})$ such that $\Psi \rtimes P \circ i_{\mathcal{K}}=\Psi$, and $\mathcal{N} \mathcal{T}_{\mathcal{L}}(\mathcal{K})=C^{*}\left(i_{\mathcal{K}}(\mathcal{K})\right)$. We call $\mathcal{N} \mathcal{T}_{\mathcal{L}}(\mathcal{K})$ the Nica-Toeplitz algebra of $\mathcal{K}$. We write $\mathcal{N} \mathcal{T}(\mathcal{L})$ for the Nica-Toeplitz algebra $\mathcal{N} \mathcal{T}_{\mathcal{L}}(\mathcal{L})$ associated to $\mathcal{L}$, viewed as a well-aligned ideal in itself (in particular, in this paper we assume that $\mathcal{L}$ satisfies the analogue of (1.8)). By (1.7) and [19, Lemma 11.1] we have a natural embedding

$$
\mathcal{N} \mathcal{T}_{\mathcal{L}}(\mathcal{K}) \hookrightarrow \mathcal{N} \mathcal{T}(\mathcal{L})
$$

The Fock representation of $\mathcal{K}$ constructed in [19] is a direct sum of Nica covariant representations. By [19, Proposition 7.6] and (1.8), here we may use the $e$-th summand of it. We recall the relevant construction. For $s \in P$, the space $X_{s}:=\mathcal{K}(s, e)$ is naturally equipped with the structure of a right Hilbert module over $A:=\mathcal{K}(e, e)$ inherited from $C^{*}$-precategory structure of $\mathcal{K}$ : we put $x \cdot a:=x a,\langle x, y\rangle:=x^{*} y$, for $x, y \in X_{s}, a \in A$. Thus we may consider the direct sum Hilbert $A$-module: $\mathcal{F}_{\mathcal{K}}:=\bigoplus_{s \in P} X_{s}$. By [19, Remark 4.3 and Proposition 5.2] we have an injective Nica covariant representation $\mathbb{L}: \mathcal{K} \rightarrow \mathcal{L}\left(\mathcal{F}_{\mathcal{K}}\right)$, there denoted $T^{e}$, determined by

$$
\mathbb{L}_{p, q}(a) x= \begin{cases}\left(a \otimes 1_{q^{-1} s}\right) x & \text { if } s \in q P, \\ 0 & \text { otherwise }\end{cases}
$$


for $a \in \mathcal{K}(p, q), x \in X_{s}$ and $p, q, s \in P$. We call $\mathbb{L}$ given by (1.10) the Fock representation of $\mathcal{K}$. The reduced Nica-Toeplitz algebra of $\mathcal{K}$ is the $C^{*}$-algebra $\mathcal{N} \mathcal{T}_{\mathcal{L}}^{r}(\mathcal{K}):=C^{*}(\mathbb{L}(\mathcal{K}))$. When $\mathcal{K}=\mathcal{L}$, we also write $\mathcal{N} \mathcal{T}^{r}(\mathcal{L}):=\mathcal{N} \mathcal{T}_{\mathcal{L}}^{r}(\mathcal{L})$. By (1.8) and [19, Proposition 7.6], the $C^{*}$-algebra $\mathcal{N} \mathcal{T}_{\mathcal{L}}^{r}(\mathcal{K})$ defined above is naturally isomorphic to the one introduced in [19, Definition 5.3]. Hence the two definitions are consistent. We refer to $\mathbb{L} \rtimes P: \mathcal{N} \mathcal{T}_{\mathcal{L}}(\mathcal{K}) \rightarrow \mathcal{N} \mathcal{T}_{\mathcal{L}}^{r}(\mathcal{K})$ as the regular representation of $\mathcal{N} \mathcal{T}_{\mathcal{L}}(\mathcal{K})$. We say that $\mathcal{K}$ is amenable when $\mathbb{L} \rtimes P$ is an isomorphism. A number of amenability criteria are given in [19, Section 8].

\section{2. $C^{*}$-ALGEBRAS ASSOCIATED TO PRODUCT SYSTEMS}

In this section we construct and analyze a canonical right-tensor $C^{*}$-precategory associated to an arbitrary product system $X$. We employ it to prove the announced uniqueness results.

2.1. Right tensor $C^{*}$-precategories associated to product systems. Let $X$ be a product system over a semigroup $P$ with coefficients in a $C^{*}$-algebra $A$. We will associate to $X$ a right-tensor $C^{*}$-precategory. In the case $P=\mathbb{N}$, it was constructed in [17, Example 3.2], and in the case $P$ is arbitrary, but the product system $X$ is regular, it was introduced in [20, Definition 3.1]. For $p, q \in P$ we put

$$
\mathcal{L}_{X}(p, q):= \begin{cases}\mathcal{L}\left(X_{q}, X_{p}\right), & \text { if } p, q \in P \backslash\{e\}, \\ \mathcal{K}\left(X_{q}, X_{p}\right), & \text { otherwise }\end{cases}
$$

With operations inherited from the corresponding spaces, $\mathcal{L}_{X}$ forms a $C^{*}$-precategory. The reason for considering smaller spaces than $\mathcal{L}\left(X_{q}, X_{p}\right)$ when $p$ or $q$ is the unit $e$ is that in general it is not clear how to define right tensoring on such spaces, cf. Remark 2.3 below. On the other hand, using the isomorphism (1.2), for all $p, q \in P$, we have the following isomorphisms of $C^{*}$-correspondences over $A$ :

$$
\mathcal{L}_{X}(p, e) \cong X_{p}, \quad \mathcal{L}_{X}(e, q) \cong \widetilde{X}_{q}
$$

where $\widetilde{X}_{q}$ is a (left) $C^{*}$-correspondence dual to $X_{q}$. In particular, $\mathcal{L}_{X}(e, e)=A$.

We will describe a right tensoring structure on $\mathcal{L}_{X}$ by introducing a family of mappings $\iota_{p, q}^{p r, q r}: \mathcal{L}\left(X_{q}, X_{p}\right) \rightarrow \mathcal{L}\left(X_{q r}, X_{p r}\right), p, q, r \in P$, which extends the standard family of diagonal homomorphisms $\iota_{q}^{q p}$, see (1.1). If $q \neq e$ we put

$$
\iota_{p, q}^{p r, q r}(T)(x y):=(T x) y, \quad \text { where } x \in X_{q}, y \in X_{r} \text { and } T \in \mathcal{L}\left(X_{q}, X_{p}\right) .
$$

Note that under the canonical isomorphisms $X_{q r} \cong X_{q} \otimes_{A} X_{r}$ and $X_{p r} \cong X_{p} \otimes_{A} X_{r}$, the operator $\iota_{p, q}^{p r, q r}(T)$ corresponds to $T \otimes 1_{r}$, where $1_{r}$ is the identity in $\mathcal{L}\left(X_{r}\right)$, and in particular $\iota_{p, q}^{p r, q r}(T) \in \mathcal{L}\left(X_{q r}, X_{p r}\right)$. In the case $q=e$, using (1.2), the formula

$$
\iota_{p, e}^{p r, r}\left(t_{x}\right)(y):=x y, \quad \text { where } y \in X_{r} \text { and } t_{x} \in \mathcal{K}\left(X_{e}, X_{p}\right), x \in X_{p},
$$

yields a well defined map. As above, under natural identifications, the operator $\iota_{p, e}^{p r, r}\left(t_{x}\right)$ corresponds to $t_{x} \otimes 1_{r} \in \mathcal{L}\left(X_{e} \otimes_{A} X_{r}, X_{p} \otimes_{A} X_{r}\right)$ and therefore $\iota_{p, e}^{p r, r}\left(t_{x}\right) \in \mathcal{L}\left(X_{r}, X_{p r}\right)$. Note that $\iota_{p, p}^{p r, p r}=\iota_{p}^{p r}$.

Proposition 2.1. The linear maps $\iota_{p, q}^{p r, q r}: \mathcal{L}_{X}(p, q) \rightarrow \mathcal{L}_{X}(p r, q r), p, q, r \in P$, yield a right tensoring on the $C^{*}$-precategory $\mathcal{L}_{X}$. We write

$$
T \otimes 1_{r}:=\iota_{p, q}^{p r, q r}(T), \quad T \in \mathcal{L}_{X}(p, q), p, q \in P .
$$

Proof. It suffices to check that $\iota_{p, q}^{p r, q r}(T)^{*}=\iota_{q, p}^{q r, p r}\left(T^{*}\right), \iota_{p, q}^{p r, q r}(T) \iota_{q, s}^{q r, s r}(S)=\iota_{p, s}^{p r, s r}(T S)$, and $\iota_{p r, q r}^{p r s, q r s}\left(\iota_{p, q}^{p r, q r}(T)\right)=\iota_{p, q}^{p r s, q r s}(T)$, for all $T \in \mathcal{L}_{X}\left(X_{q}, X_{p}\right), S \in \mathcal{L}_{X}\left(X_{s}, X_{q}\right), p, q, r, s \in P$. Viewing operators $\iota_{p, q}^{p r, q r}(T)$ as $T \otimes 1_{r}$, see discussion above, this is straightforward. 
Definition 2.2. We call the pair $\left(\mathcal{L}_{X},\left\{\otimes 1_{r}\right\}_{r \in P}\right)$ constructed above, the right-tensor $C^{*}$ precategory associated to the product system $X$. We also put

$$
\mathcal{K}_{X}(p, q):=\mathcal{K}\left(X_{q}, X_{p}\right) \quad p, q \in P .
$$

Clearly, $\mathcal{K}_{X}:=\left\{\mathcal{K}_{X}(p, q)\right\}_{p, q \in P}$ is an essential ideal in the $C^{*}$-precategory $\mathcal{L}_{X}$.

Remark 2.3. If each $C^{*}$-correspondence $X_{p}, p \in P$, is left essential (which is automatic when $\left.P^{*} \neq\{e\}\right)$ then the formula

$$
\left(T \otimes 1_{r}\right)(a x):=T(a) x, \quad T \in \mathcal{L}\left(A, X_{p}\right), a \in A, x \in X_{r}, p, r \in P,
$$

allows one to extend the right tensoring from $\mathcal{L}_{X}$ on the whole $C^{*}$-category $\left\{\mathcal{L}\left(X_{q}, X_{p}\right)\right\}_{p, q \in P}$. Note that $\mathcal{L}_{X}$ is a $C^{*}$-category if and only if $A$ is unital, if and only if $\mathcal{L}_{X}=\left\{\mathcal{L}\left(X_{q}, X_{p}\right)\right\}_{p, q \in P}$.

Lemma 2.4. The ideal $\mathcal{K}_{X}$ in the right-tensor $C^{*}$-precategory $\left(\mathcal{L}_{X},\left\{\otimes 1_{r}\right\}_{r \in P}\right)$ associated to the product system $X$ is $\otimes 1$-nondegenerate. Moreover, $\mathcal{K}_{X} \otimes 1 \subseteq \mathcal{K}_{X}$ if and only if $\phi_{p}(A) \subseteq$ $\mathcal{K}\left(X_{p}\right)$ for every $p \in P$.

Proof. Let $x, y, z \in X_{p}, u \in X_{r}$ and $v \in X_{p r}$ for some $p, r \in P, p \neq e$. Then $\left(\Theta_{x, y} \otimes 1_{r}\right) \Theta_{z u, v}=$ $\Theta_{x\langle y, z\rangle_{X_{p}} u, v}$. Since elements of the form $x\langle y, z\rangle_{X_{p}}$ span $X_{p}$ and since $X_{p} X_{r}=X_{p r}$, we conclude that elements $\left(\Theta_{x, y} \otimes 1_{r}\right) \Theta_{z u, v}$ span $\mathcal{K}\left(X_{p r}\right)$. Hence $\mathcal{K}_{X}$ is $\otimes 1$-nondegenerate. It is clear that $\mathcal{K}_{X} \otimes 1 \subseteq \mathcal{K}_{X}$ implies $\phi_{p}(A)=A \otimes 1_{p} \subseteq \mathcal{K}\left(X_{p}\right)$ for $p \in P$. The converse implication follows from the standard fact [24, Proposition 4.7], cf. [20, Lemma 3.2].

Remark 2.5. We claim that $\mathcal{K}_{X}$ and $A$ may be regarded as being Morita equivalent as $C^{*}$ precategories, see also Lemma 2.6. To make this more precise, we introduce some notation first. If $Z$ and $Y$ are two right Hilbert $A$-modules and $I$ is an ideal in $A$, we let $\mathcal{L}_{I}(Z, Y):=$ $\{a \in \mathcal{L}(Z, Y): a(Z) \subseteq Y I\}$. Note that $a(Z) \subseteq Y I$ is equivalent to $a^{*}(Y) \subseteq Z I$. We also put $\mathcal{K}_{I}(Z, Y):=\mathcal{L}_{I}(Z, Y) \cap \mathcal{K}(Z, Y)$, cf. [17, Lemmas 1.1 and 1.2]. Now, given a product system $X$ over $P$, for any ideal $J$ in $A$ the formulas

$$
\mathcal{K}_{X}(J):=\left\{\mathcal{K}_{J}\left(X_{q}, X_{p}\right)\right\}_{p, q \in P}, \quad \mathcal{L}_{X}(J):=\left\{\mathcal{L}_{J}\left(X_{q}, X_{p}\right)\right\}_{p, q \in P}
$$

define ideals in $\mathcal{L}_{X}=\left\{\mathcal{L}\left(X_{q}, X_{p}\right)\right\}_{p, q \in P}$, as the reader may readily verify. We claim that every ideal in $\mathcal{K}_{X}=\left\{\mathcal{K}\left(X_{q}, X_{p}\right)\right\}_{p, q \in P}$ is of the form $\mathcal{K}_{X}(J)$ for some ideal $J$ in $A$. Indeed, this is proved in [17, Proposition 2.17] in the case $P=\mathbb{N}$ but the proof works for general $P$.

Lemma 2.6. We have a one-to-one correspondence, established by the formula

$$
\Psi_{p, q}\left(\Theta_{x, y}\right)=\psi_{p}(x) \psi_{q}(y)^{*} \quad \text { for } x \in X_{p}, y \in X_{q}, p, q \in P,
$$

between representations $\Psi=\left\{\Psi_{p, q}\right\}_{p, q \in P}$ of $\mathcal{K}_{X}$ and families $\psi=\left\{\left(\psi_{e}, \psi_{p}\right)\right\}_{p \in P}$ where, for each $p \in P,\left(\psi_{e}, \psi_{p}\right)$ is a representation of the Hilbert A-module $X_{p}$.

Moreover, if $\Psi$ is a representation of $\mathcal{K}_{X}$ on a Hilbert space $H$ and $\bar{\Psi}$ is its extension to $\mathcal{L}_{X}$ determined by (1.3), then with $\psi_{e}=\Psi_{e, e}$ we have

$$
\operatorname{ker} \Psi=\mathcal{K}_{X}\left(\operatorname{ker} \psi_{e}\right) \quad \text { and } \quad \operatorname{ker} \bar{\Psi}=\mathcal{L}_{X}\left(\operatorname{ker} \psi_{e}\right) .
$$

Proof. Let $\psi=\left\{\left(\psi_{e}, \psi_{p}\right)\right\}_{p \in P}$ be a family of representations of right-Hilbert modules $X_{p}$ for $p \in P$ in a $C^{*}$-algebra $B$. Let $p, q, r \in P$. It is well known that (2.1) determines uniquely a linear contraction $\Psi_{p, q}: \mathcal{K}\left(X_{q}, X_{p}\right) \longrightarrow B$ which is isometric if $\psi_{e}$ is injective, see for instance [16, Lemma 2.2, Remark 2.3]. It is straightforward to see that the relations

$$
\Psi_{p, q}(S)^{*}=\Psi_{q, p}\left(S^{*}\right) \quad \text { and } \quad \Psi_{p, q}(S) \psi_{q, r}(T)=\psi_{p, r}(S T)
$$

hold for 'rank one' operators $S=\Theta_{x, y}, T=\Theta_{u, w}$. Hence these formulas hold for arbitrary $S \in \mathcal{K}\left(X_{q}, X_{p}\right), T \in \mathcal{K}\left(X_{r}, X_{q}\right)$. Thus $\Psi=\left\{\Psi_{p, q}\right\}_{p, q \in P}$ is a representation of $\mathcal{K}_{X}$. 
If now $\Psi:=\left\{\Psi_{p, q}\right\}_{p, q \in P}$ is an arbitrary representation of $\mathcal{K}_{X}$ in a $C^{*}$-algebra $B$ then by (1.2) we can define a family of maps $\psi_{p}: X_{p} \rightarrow B$ by $\psi_{p}(x):=\Psi_{p, e}\left(t_{x}\right), x \in X_{p}, p \in P$. A routine verification shows that $\left(\psi_{e}, \psi_{p}\right): X_{p} \rightarrow B$ is a right-Hilbert module representation.

The equality ker $\Psi=\mathcal{K}_{X}\left(\operatorname{ker} \psi_{e}\right)$ follows from Remark 2.5, as $\mathcal{K}_{X}\left(\operatorname{ker} \psi_{e}\right)(e, e)=\operatorname{ker} \psi_{e}=$ $(\operatorname{ker} \Psi)(e, e)$. By [19, Proposition 2.13] we have $(\operatorname{ker} \bar{\Psi})(p, q)=\left\{a \in \mathcal{L}_{X}(p, q): a \mathcal{K}_{X}(q, q) \subseteq\right.$ $\left.\operatorname{ker} \Psi_{p, q}\right\}$. Thus the inclusion $\mathcal{L}_{X}\left(\operatorname{ker} \psi_{e}\right)(p, q) \subseteq(\operatorname{ker} \bar{\Psi})(p, q)$ is immediate. For the reverse let $a \in(\operatorname{ker} \bar{\Psi})(p, q)$ and $x \in X_{q}$. Note that $x$ may be written as $x=b x^{\prime}$ where $b \in \mathcal{K}\left(X_{q}\right)$ and $x^{\prime} \in X_{q}$. Hence $a x=(a b) x^{\prime} \in \mathcal{K}_{X}(p, q) x^{\prime} \subseteq X_{p}\left(\operatorname{ker} \psi_{e}\right)$, and $a \in \mathcal{L}_{X}\left(\operatorname{ker} \psi_{e}\right)(p, q)$.

Remark 2.7. The semigroup operation in $P$ is irrelevant for the assertions in Remark 2.5 and Lemma 2.6 - they remain true when $P$ is any set with a distinguished element $e \in P$ and $\left\{X_{p}\right\}_{p \in S}$ is a family of right Hilbert modules over a $C^{*}$-algebra $A$ such that $X_{e}=A_{A}$.

Proposition 2.8. Let $X$ be a product system over an arbitrary semigroup $P$. The bijective correspondence in Lemma 2.6 restricts to a one-to-one correspondence between representations $\psi=\left\{\psi_{p}\right\}_{p \in P}$ of $X$ and right-tensor representations $\Psi:=\left\{\Psi_{p, q}\right\}_{p, q \in P}$ of $\mathcal{K}_{X}$. In particular, $\mathcal{T}(X)$ is isomorphic to $\mathcal{T}_{\mathcal{L}_{X}}\left(\mathcal{K}_{X}\right)$, the Toeplitz algebra of $\mathcal{K}_{X}$.

Proof. Let $\psi$ and $\Psi$ be the corresponding objects in Lemma 2.6. Suppose first that $\Psi$ is a righttensor representation of $\mathcal{K}_{X}$. For any $x \in X_{p}$ and $y \in X_{q}, p, q \in P$, we have $\left(t_{x} \otimes 1_{y}\right) y=t_{x y}$. Thus

$$
\psi_{p}(x) \psi_{q}(y)=\Psi_{p, e}\left(t_{x}\right) \Psi_{q, e}\left(t_{y}\right)=\Psi_{p q, e}\left(\left(t_{x} \otimes 1_{y}\right) t_{y}\right)=\psi_{p q}(x),
$$

so $\psi=\left\{\psi_{p}\right\}_{p \in P}$ is a representation of the product system $X$. Suppose now that $\psi$ is a representation of $X$. Let $p, q, s, t \in P$ with $s \geq q$. Consider $S=\Theta_{x, y} \in \mathcal{K}\left(X_{q}, X_{p}\right)$ and $T=\Theta_{u^{\prime} u, w} \in \mathcal{K}\left(X_{t}, X_{s}\right)$ where $u^{\prime} \in X_{q}$ and $u \in X_{q^{-1} s}$. Then

$$
\begin{aligned}
\Psi_{p, q}(S) \Psi_{s, t}(T) & =\psi(x) \psi(y)^{*} \psi\left(u^{\prime} u\right) \psi(w)^{*}=\psi\left(x\left\langle y, u^{\prime}\right\rangle_{A} u\right) \psi(w)^{*}=\Psi_{p q^{-1}, t}\left(\Theta_{x\left\langle y, u^{\prime}\right\rangle_{A} u, w}\right) \\
& =\Psi_{p q^{-1}, t}\left(\iota_{p, q}^{p q^{-1} s, s}\left(\Theta_{x, y}\right) \Theta_{u^{\prime} u, w}\right)=\Psi_{p q^{-1}, t}\left(\left(S \otimes 1_{q^{-1} s}\right) T\right) .
\end{aligned}
$$

Hence, by linearity and continuity, $\Psi$ is a right-tensor representation.

2.2. $C^{*}$-algebras associated with product systems over LCM semigroups. For the remaining of this section we assume that $P$ is a right LCM semigroup.

Lemma 2.9. A product system $X$ over $P$ is compactly-aligned if and only if the ideal $\mathcal{K}_{X}$ is well-aligned in the associated right-tensor $C^{*}$-precategory $\left(\mathcal{L}_{X},\left\{\otimes 1_{r}\right\}_{r \in P}\right)$. In particular, $\mathcal{K}_{X}$ satisfies (1.7) and (1.8).

Proof. $\mathcal{K}_{X}$ satisfies (1.7) by Lemma 2.4. The remaining claims are immediate.

The next proposition generalizes [17, Proposition 3.14] from $\mathbb{N}$ to right LCM semigroups.

Proposition 2.10. If $X$ is a compactly-aligned product system over a right LCM semigroup $P$, then the bijective correspondence in Proposition 2.8 preserves Nica covariance of representations and, hence, it gives rise to a canonical isomorphism

$$
\mathcal{N} \mathcal{T}_{\mathcal{L}_{X}}\left(\mathcal{K}_{X}\right) \cong \mathcal{N} \mathcal{T}(X)
$$

Proof. If $\Psi:=\left\{\Psi_{p, q}\right\}_{p, q \in P}$ is a Nica covariant representation of $\mathcal{K}_{X}$, then it is also a righttensor representation and therefore $\psi=\left\{\psi_{p}\right\}_{p \in P}$ is a representation of the product system $X$. Since $\psi^{(p)}=\Psi_{p, p}$ and $\iota_{p}^{p q}(S)=S \otimes 1_{q}$, for $S \in \mathcal{K}\left(X_{p}\right)$ and $p, q \in P$, Nica covariance of $\Psi$ implies Nica covariance of $\psi$. 
Let $\psi=\left\{\psi_{p}\right\}_{p \in P}$ be a Nica covariant representation of $X$ on a Hilbert space $H$. Let $\Psi=\left\{\Psi_{p, q}\right\}_{p, q \in P}$ be the representation of $\mathcal{K}_{X}$ given by (2.1). To see that $\Psi$ is Nica covariant, let $S=\Theta_{x, y} \in \mathcal{K}\left(X_{q}, X_{p}\right)$ and $T=\Theta_{u, w} \in \mathcal{K}\left(X_{t}, X_{s}\right)$ where $p, q, s, t \in P$. Express $y=Y y^{\prime}$ with $Y \in \mathcal{K}\left(X_{q}\right)$ and $y^{\prime} \in X_{q}$, and similarly $u=U u^{\prime}$ with $U \in \mathcal{K}\left(X_{s}\right)$ and $u^{\prime} \in X_{s}$. Then $\psi(y)^{*} \psi(u)=\psi\left(y^{\prime}\right)^{*} \psi^{(q)}\left(Y^{*}\right) \psi^{(s)}(U) \psi\left(u^{\prime}\right)$. Therefore, by Nica covariance of $\psi$, if $q P \cap s P=\emptyset$, then $\psi(y)^{*} \psi(u)=0$ and hence $\Psi_{p, q}(S) \Psi_{s, t}(T)=0$. Assume that $q P \cap s P=r P$, for some $r \in P$. Again by Nica covariance of $\psi$ we get

$$
\psi(y)^{*} \psi(u)=\psi\left(y^{\prime}\right)^{*} \psi^{(r)}\left(\left(Y^{*} \otimes 1_{q^{-1} r}\right)\left(U \otimes 1_{s^{-1} r}\right)\right) \psi\left(u^{\prime}\right)
$$

We claim that $\psi(y)^{*} \psi(u) \in \Psi_{q^{-1} r, s^{-1} r}\left(\mathcal{K}\left(X_{s^{-1} r}, X_{q^{-1} r}\right)\right.$. Indeed, the operator $\psi^{(r)}\left(\left(Y^{*} \otimes\right.\right.$ $\left.\left.1_{q^{-1} r}\right)\left(U \otimes 1_{q^{-1} s}\right)\right)$ can be approximated by finite sums of elements of the form $\psi_{r}\left(v^{\prime} v\right) \psi_{r}\left(z^{\prime} z\right)^{*}$ where $v^{\prime} \in X_{q}, v \in X_{q^{-1} r}$, and $z^{\prime} \in X_{s}, z \in X_{s^{-1} r}$. Since

$$
\psi_{q}\left(y^{\prime}\right)^{*} \psi_{r}\left(v^{\prime} v\right) \psi_{r}\left(z^{\prime} z\right)^{*} \psi_{s}\left(u^{\prime}\right)=\psi_{q^{-1} r}\left(\left\langle y^{\prime}, v^{\prime}\right\rangle_{q} v\right) \psi_{s^{-1} r}\left(\left\langle u^{\prime}, z^{\prime}\right\rangle_{s} z\right)^{*}
$$

is an element of $\Psi_{q^{-1} r, s^{-1} r}\left(\mathcal{K}\left(X_{s^{-1} r}, X_{q^{-1} r}\right)\right)$, so is $\psi(y)^{*} \psi(u)$. Accordingly,

$$
\Psi_{p, q}(S) \Psi_{s, t}(T)=\psi_{p}(x) \psi_{q}(y)^{*} \psi_{s}(u) \psi_{t}(w)^{*} \in \Psi_{p q^{-1} r, t s^{-1} r}\left(\mathcal{K}\left(X_{t s^{-1} r}, X_{p q^{-1} r}\right)\right) .
$$

Hence the product $\Psi_{p, q}(S) \Psi_{s, t}(T)$ acts as zero on the orthogonal complement of the space $H_{t s^{-1} r}:=\overline{\psi_{t s^{-1} r}\left(X_{t s^{-1} r}\right) H}$. Clearly, the same is true for the operator $\Psi_{p q^{-1} r, t s^{-1} r}((S \otimes$ $\left.\left.1_{q^{-1} r}\right)\left(T \otimes 1_{s^{-1} r}\right)\right)$. Consider an element $\psi_{t^{-1} r}\left(w_{0} u_{0}\right) h$ where $w_{0} \in X_{t}, u_{0} \in X_{s^{-1} r}, h \in H$. The linear span of such elements is in $H_{t s^{-1} r}$ and we have

$$
\begin{aligned}
\Psi_{s, t}(T) \psi_{t s^{-1} r}\left(w_{0} u_{0}\right) & =\psi_{s}(u) \psi_{t}(w)^{*} \psi_{t}\left(w_{0}\right) \psi_{s^{-1} r}\left(u_{0}\right)=\psi_{r}\left(u\left\langle w, w_{0}\right\rangle_{t} u_{0}\right) \\
& =\psi_{r}\left(\left(\Theta_{u, w} \otimes 1_{s^{-1} r}\right) w_{0} u_{0}\right)=\Psi_{r, t s^{-1} r}\left(T \otimes 1_{s^{-1} r}\right) \psi_{t s^{-1} r}\left(w_{0} u_{0}\right) .
\end{aligned}
$$

Hence $\Psi_{s, t}(T)$ and $\Psi_{r, t s^{-1} r}\left(T \otimes 1_{s^{-1} r}\right)$ coincide on the space $H_{t s^{-1} r}$, and they map this space into the space $H_{r}:=\overline{\psi_{r}\left(X_{r}\right) H}$. Consider an element $\psi_{r}\left(y_{0} x_{0}\right) h$ where $x_{0} \in X_{q^{-1} r}, y_{0} \in X_{q}$, $h \in H$. The linear span of such elements is in $H_{r}$ and we have

$$
\begin{aligned}
\Psi_{p, q}(S) \psi_{r}\left(y_{0} x_{0}\right) & =\psi_{p}(x) \psi_{q}(y)^{*} \psi_{q}\left(y_{0}\right) \psi_{q^{-1} r}\left(x_{0}\right)=\psi_{p q^{-1} r}\left(x\left\langle y, y_{0}\right\rangle_{q} u_{0}\right) \\
& =\psi_{r}\left(\left(\Theta_{x, y} \otimes 1_{q^{-1} r}\right) y_{0} x_{0}\right)=\Psi_{p q^{-1} r, r}\left(S \otimes 1_{q^{-1} r}\right) \psi_{r}\left(y_{0} x_{0}\right) .
\end{aligned}
$$

Hence $\Psi_{p, q}(S)$ and $\Psi_{p q^{-1} r, r}\left(S \otimes 1_{q^{-1} r}\right)$ coincide when restricted to $H_{r}$. Combining these two observations we get

$$
\begin{aligned}
\Psi_{p, q}(S) \Psi_{s, t}(T) & =\Psi_{p q^{-1} r, r}\left(S \otimes 1_{q^{-1} r}\right) \Psi_{r, t s^{-1} r}\left(T \otimes 1_{s^{-1} r}\right) \\
& =\Psi_{p q^{-1} r, t s^{-1} r}\left(\left(S \otimes 1_{q^{-1} r}\right)\left(T \otimes 1_{s^{-1} r}\right)\right) .
\end{aligned}
$$

Thus $\Psi:=\left\{\Psi_{p, q}\right\}_{p, q \in P}$ is Nica covariant.

The above result motivates the following definition.

Definition 2.11. Let $X$ be a compactly-aligned product system over a right LCM semigroup $P$. We let $\mathcal{N} \mathcal{T}^{r}(X):=\mathcal{N} \mathcal{T}_{\mathcal{L}_{X}}^{r}\left(\mathcal{K}_{X}\right)$ and call it the reduced Nica-Toeplitz algebra of $X$. We also put

$$
\mathcal{D} \mathcal{R}(\mathcal{N} \mathcal{T}(X)):=\mathcal{N} \mathcal{T}\left(\mathcal{L}_{X}\right) \quad \text { and } \quad \mathcal{D R}^{r}(\mathcal{N} \mathcal{T}(X)):=\mathcal{N} \mathcal{T}^{r}\left(\mathcal{L}_{X}\right)
$$

$\left(\mathcal{D} \mathcal{R}\right.$ stands for Doplicher-Roberts). We denote by $\bar{\Lambda}: \mathcal{D} \mathcal{R}(\mathcal{N} \mathcal{T}(X)) \rightarrow \mathcal{D R}^{r}(\mathcal{N} \mathcal{T}(X))$ and $\Lambda: \mathcal{N} \mathcal{T}(X) \rightarrow \mathcal{N} \mathcal{T}^{r}(X)$ the canonical epimorphisms. 
Let $X$ be a compactly-aligned product system. In [11] Fowler constructed the Fock representation $l: X \rightarrow \mathcal{L}(\mathcal{F}(X))$ of $X$. The Fock spaces for $X, \mathcal{K}_{X}$ and $\mathcal{L}_{X}$ coincide and are equal to the Hilbert $A$-module direct sum $\mathcal{F}(X)=\bigoplus_{p \in P} X_{p}$. The Fock representation $\overline{\mathbb{L}}: \mathcal{L}_{X} \rightarrow \mathcal{L}(\mathcal{F}(X))$ of $\mathcal{L}_{X}$ is an extension of the Fock representation $\mathbb{L}: \mathcal{K}_{X} \rightarrow \mathcal{L}(\mathcal{F}(X))$ of $\mathcal{K}_{X}$ which is in turn an extension of $l$. This in particular leads to the inclusion

$$
\mathcal{N} \mathcal{T}^{r}(X)=\overline{\operatorname{span}}\left\{l(x) l(y)^{*}: x, y \in X\right\} \subseteq \mathcal{D} \mathcal{R}^{r}(\mathcal{N} \mathcal{T}(X))
$$

By [19, Lemma 11.1], there is a commutative diagram

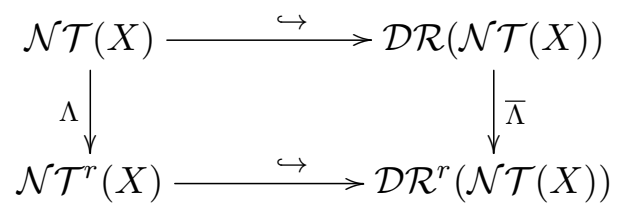

in which the horizontal maps are embeddings. The map $\bar{\Lambda}$ is injective on the core subalgebra $B_{e}^{i \mathcal{L}_{X}}=\overline{\operatorname{span}}\left\{i_{\mathcal{L}_{X}}(a): a \in \mathcal{L}_{X}(p, p), p \in P\right\}$ of $\mathcal{D} \mathcal{R}(\mathcal{N} \mathcal{T}(X))$ and $\Lambda$ is injective on

$$
B_{e}^{X}:=B_{e}^{i_{\mathcal{K}_{X}}}=\overline{\operatorname{span}}\left\{i_{X}(x) i_{X}(y)^{*}: x, y \in X, d(x)=d(y)\right\}
$$

see [19, Corollary 6.4]. Clearly, $B_{e}^{X} \subseteq B_{e}^{i_{\mathcal{L}_{X}}}$. We will characterize representations of $X$ that give rise to injective representations of $B_{e}^{X}$ and $B_{e}^{i \mathcal{L}_{X}}$ respectively. To this end, we introduce canonical projections associated to a representation of $X$.

Definition 2.12. If $\psi: X \rightarrow \mathcal{B}(H)$ is a representation of a compactly-aligned product system $X$, for every $p \in P$ we denote by $Q_{p}^{\psi} \in \mathcal{B}(H)$ the projection such that

$$
Q_{p}^{\psi} H= \begin{cases}\psi^{(p)}\left(\mathcal{K}\left(X_{p}\right)\right) H & \text { if } p \in P \backslash\{e\}, \\ \hline \psi\left(X_{e}\right) H & \text { if } p=e .\end{cases}
$$

Remark 2.13. If $\Psi:=\left\{\Psi_{p, q}\right\}_{p, q \in P}$ is the representation of $\mathcal{K}_{X}$ given by Proposition 2.8, then $Q_{p}^{\psi}$ equals the projection $Q_{p}^{\Psi}$ associated to $\Psi$ in $[19$, Definition 9.1] for $p \in P$. In particular, if $\bar{\Psi}:=\left\{\bar{\Psi}_{p, q}\right\}_{p, q \in P}$ is the extension of $\Psi$ to $\mathcal{L}_{X}$, then $Q_{p}^{\Phi}=\bar{\Psi}_{p, q}\left(i_{\mathcal{L}}\left(1_{p}\right)\right)$, for all $p \in P \backslash\{e\}$.

Lemma 2.14. A representation $\psi: X \rightarrow \mathcal{B}(H)$ is Nica covariant if and only if the projections $\left\{Q_{p}^{\psi}\right\}_{p \in P} \in \mathcal{B}(H)$ satisfy the Nica covariance relation

$$
Q_{p}^{\psi} Q_{q}^{\psi}= \begin{cases}Q_{r}^{\psi}, & \text { if } q P \cap s P=r P \text { for some } r \in P, \\ 0, & \text { if } q P \cap s P=\emptyset .\end{cases}
$$

Moreover, if $\psi: X \rightarrow \mathcal{B}(H)$ is Nica covariant, the representation $\psi \rtimes P: \mathcal{N} \mathcal{T}(X) \rightarrow B(H)$ extends uniquely to a representation $\overline{\psi \rtimes P}$ of $\mathcal{D} \mathcal{R}(\mathcal{N} \mathcal{T}(X))$ such that

$$
(\overline{\psi \rtimes P})\left(i_{\mathcal{L}_{X}}(a)\right)=Q_{p}^{\psi}(\overline{\psi \rtimes P})\left(i_{\mathcal{L}_{X}}(a)\right) Q_{q}^{\psi}, \quad a \in \mathcal{L}\left(X_{p}, X_{q}\right), p, q \in P \backslash\{e\} .
$$

In fact, $\overline{\psi \rtimes P}=\bar{\Psi} \rtimes P$ where $\Psi:=\left\{\Psi_{p, q}\right\}_{p, q \in P}$ is the associated representation of $\mathcal{K}_{X}$.

Proof. If $\psi: X \rightarrow \mathcal{B}(H)$ is Nica covariant then (2.2) holds by [19, Propositions 9.4 and 9.7]. Conversely, if $(2.2)$ holds then for $a \in \mathcal{K}\left(X_{p}\right)$ and $b \in \mathcal{K}\left(X_{q}\right)$ the product $\psi^{(p)}(a) \psi^{(q)}(b)$ equals $\psi^{(p)}(a) Q_{p}^{\psi} Q_{q}^{\psi} \psi^{(q)}(b)$, which is zero if $p P \cap q P=\emptyset$ or is $\psi^{(p)}(a) Q_{r}^{\psi} Q_{q}^{\psi} \psi^{(q)}(b)$ if $p P \cap q P=r P$. In the latter case, we have $Q_{r}^{\psi} \leq Q_{q}^{\psi}$ and so by [19, Proposition 9.4] applied to $\bar{\Psi}:=\left\{\bar{\Psi}_{p, q}\right\}_{p, q \in P}$, we obtain $\psi^{(p)}(a) Q_{r}^{\psi} \psi^{(q)}(b)=\psi^{(r)}\left(\iota_{p}^{r}(a) \iota_{q}^{r}(b)\right)$, using also that $\iota_{p}^{r}(a) \iota_{q}^{r}(b) \in \mathcal{K}_{X}(r, r)$. 
If $\psi: X \rightarrow \mathcal{B}(H)$ is Nica covariant, then $\bar{\Psi}:=\left\{\bar{\Psi}_{p, q}\right\}_{p, q \in P}$ is Nica covariant by [19, Proposition 9.5]. Putting $\overline{\psi \rtimes P}:=\bar{\Psi} \rtimes P$ for any $a \in \mathcal{L}\left(X_{p}, X_{q}\right), p, q \in P \backslash\{e\}$ we get $(\overline{\psi \rtimes P})\left(i_{\mathcal{L}_{X}}(a)\right)=\bar{\Psi}_{p, q}\left(i_{\mathcal{L}_{X}}(a)\right)$, and therefore relations $(2.3)$ are satisfied. Conversely, if $\overline{\psi \rtimes P}$ is any representation of $\mathcal{D} \mathcal{R}(\mathcal{N} \mathcal{T}(X))$ that extends $\psi \rtimes P$, we get a Nica covariant representation $\Phi$ of $\mathcal{L}_{X}$ that extends $\Psi$. The relations (2.3) imply that $\Phi=\bar{\Psi}$ and hence $\overline{\psi \rtimes P}:=\bar{\Psi} \rtimes P$.

Via the bijective correspondence in (2.1), we transport the notions of Toeplitz representation and condition $(\mathrm{C})$ for representations of $\mathcal{K}_{X}$, see [19, Definition 6.2 and Definition 10.1] to representations of the product system.

Definition 2.15. A Nica covariant representation $\psi: X \rightarrow \mathcal{B}(H)$ is Toeplitz covariant or Nica-Toeplitz covariant if for each finite family $q_{1}, \ldots, q_{n} \in P \backslash P^{*}, n \in \mathbb{N}$, we have

$$
\psi_{e}(A) \cap \overline{\operatorname{span}}\left\{\psi^{\left(q_{i}\right)}\left(\mathcal{K}\left(X_{q_{i}}\right)\right): i=1, \ldots, n\right\}=\{0\} .
$$

The representation $\psi$ satisfies condition $(C)$ if for each finite family $q_{1}, \ldots, q_{n} \in P \backslash P^{*}, n \in \mathbb{N}$,

$$
\text { the map } A \ni a \longmapsto \psi_{e}(a) \prod_{i=1}^{n}\left(1-Q_{q_{i}}^{\psi}\right) \text { is injective. }
$$

Lemma 2.16. Let $\psi: X \rightarrow \mathcal{B}(H)$ be a Nica covariant representation of a compactly-aligned product system $X$ and $\Psi:=\left\{\Psi_{p, q}\right\}_{p, q \in P}$ the associated representation of $\mathcal{K}_{X}$.

(i) $\psi$ is Toeplitz covariant if and only if $\Psi$ is Toeplitz covariant.

(ii) $\psi$ satisfies condition $(C)$ if and only if $\Psi$ satisfies condition $(C)$.

Proof. (i). Since $\psi^{(q)}\left(\mathcal{K}\left(X_{q}\right)\right)=\psi_{q}\left(X_{q}\right) \psi_{q}\left(X_{q}\right)^{*}=\Psi_{q, q}\left(\mathcal{K}\left(X_{q}\right)\right)$, Toeplitz covariance of $\Psi$ immediately implies that of $\psi$. The converse is left to the reader.

(ii). In one direction, it is immediate that $\psi$ satisfies condition (C) when $\Psi$ does. For the converse, let $p \in P$ and $q_{1}, \ldots, q_{n} \in P$ be such that $p \nsupseteq q_{i}$, for $i=1, \ldots, n$ for some $n \in \mathbb{N}$. We must show that $\mathcal{K}\left(X_{p}\right) \ni a \longrightarrow \psi^{(p)}(a) \prod_{i=1}^{n}\left(1-Q_{q_{i}}^{\psi}\right)=\Psi_{p, p}(a) \prod_{i=1}^{n}\left(1-Q_{q_{i}}^{\Psi}\right)$ is injective.

It follows from $(2.2)$ that $Q_{p}^{\psi} \prod_{i=1}^{n}\left(1-Q_{q_{i}}^{\psi}\right)=\prod_{i=1}^{k}\left(Q_{p}^{\psi}-Q_{s_{i}}^{\psi}\right)$ for some $k \geq n$ and $p \leq s_{i} \not \leq p$. Then $p^{-1} s_{i} \notin P^{*}$, for all $i=1, \ldots, k$. Hence the space $K=\prod_{i=1}^{k}\left(1-Q_{p^{-1} s_{i}}^{\psi}\right) H$ is invariant for $\psi_{e}$ and $\left.\psi_{e}\right|_{K}$ is faithful. By [13, Proposition 1.6(2)], this implies that $\overline{\psi_{p}\left(X_{p}\right) K}$ is invariant for $\psi^{(p)}$ and $\left.\psi^{(p)}\right|_{\psi_{p}\left(X_{p}\right) K}$ is faithful. For any $q \in P$, applying [19, Proposition 9.4] twice we get

$$
\psi_{p}\left(X_{p}\right) Q_{q}^{\psi}=\Psi_{p, e}\left(X_{p}\right) Q_{q}^{\Psi}=\bar{\Psi}_{p q, p}\left(X_{p} \otimes 1_{q}\right)=Q_{p q}^{\Psi} \Psi_{p, e}\left(X_{p}\right)=Q_{p q}^{\psi} \psi_{p}\left(X_{p}\right) .
$$

Therefore

$$
\psi_{p}\left(X_{p}\right) \prod_{i=1}^{k}\left(1-Q_{p^{-1} s_{i}}^{\psi}\right)=\prod_{i=1}^{k}\left(Q_{p}^{\psi}-Q_{s_{i}}^{\psi}\right) \psi_{p}\left(X_{p}\right)=Q_{p}^{\psi} \prod_{i=1}^{n}\left(1-Q_{q_{i}}^{\psi}\right) \psi_{p}\left(X_{p}\right),
$$

which gives the desired injectivity.

Theorem 2.17 (Faithfulness on core subalgebras). Let $\psi: X \rightarrow \mathcal{B}(H)$ be a Nica covariant representation of a compactly-aligned product system $X$. Let $\overline{\psi \rtimes P}: \mathcal{D} \mathcal{R}(\mathcal{N} \mathcal{T}(X)) \rightarrow B(H)$ be the extension of $\psi \rtimes P: \mathcal{N} \mathcal{T}(X) \rightarrow B(H)$ described in Lemma 2.14.

(i) $\psi \rtimes P$ is faithful on the core $B_{e}^{X}$ of $\mathcal{N} \mathcal{T}(X)$ if and only if $\psi$ is injective and Toeplitz covariant.

(ii) $\overline{\psi \rtimes P}$ is faithful on the core $B_{e}^{i \mathcal{L}_{X}}$ of $\mathcal{D} \mathcal{R}(\mathcal{N} \mathcal{T}(X))$ if and only if $\psi$ satisfies condition (C). 
Moreover, if $\phi_{p}(A) \subseteq \mathcal{K}\left(X_{p}\right)$ for every $p \in P$, then the equivalent conditions in (i) are satisfied if and only if those in (ii) hold.

Proof. Item (i) follows from Lemma 2.16(i) and [19, Corollary 6.3] applied to $\Psi$. By Lemma 2.16(ii) and [19, Corollary 10.5], $\psi$ satisfies condition (C) if and only if $\bar{\Psi}$ is Nica-Toeplitz covariant and injective. Hence (ii) follows from [19, Corollary 6.3] applied to $\bar{\Psi}$. The last claim of the theorem follows from Lemma 2.4 and [19, Proposition 10.4].

2.3. Uniqueness theorems. We aim to prove a uniqueness result for $\mathcal{N} \mathcal{T}(X)$. Our result, see Theorem 2.19, may be considered a far-reaching generalization of [13, Theorem 2.1] and [23, Theorem 3.7], and was motivated in part by the need to better understand both hypotheses and claims of [11, Theorem 7.2]. The proof will employ our abstract uniqueness theorem for $C^{*}$-algebras associated to well-aligned ideals in $C^{*}$-precategories, cf. [19, Corollary 10.14]. As spin-offs of our strategy of proof we will obtain a uniqueness result in a new context, see Theorem 2.21, and a generalization of [12, Theorem 5.1].

We start with some preparation. We recall that aperiodicity for the group of right tensoring $\left\{\otimes 1_{h}\right\}_{h \in P^{*}}$ in a $C^{*}$-precategory was introduced in [19, Definition 10.8]. The notion of aperiodic Fell bundle is from [21]. Further, for any product system $X$ the spaces $\left\{X_{h}\right\}_{h \in P^{*}}$ form a saturated Fell bundle over the discrete group of units $P^{*}$, see Remark 1.3. By [22, Theorem 9.8], $\left\{X_{h}\right\}_{h \in P^{*}}$ is aperiodic if and only if its dual action on the spectrum $\widehat{A}$ is topologically free, at least when $A$ contains an essential ideal which is separable or of Type I.

Proposition 2.18. If $X$ is a product system over a semigroup $P$, then the group $\left\{\otimes 1_{h}\right\}_{h \in P^{*}}$ of automorphisms of $\mathcal{K}_{X}$ is aperiodic if and only if the Fell bundle $\left\{X_{h}\right\}_{h \in P^{*}}$ is aperiodic.

Proof. The only if part is trivial as $X_{h}=\mathcal{K}_{X}(h, e), h \in P^{*}$. For the converse, let $p \in$ $P$ and $h \in P^{*} \neq\{e\}$. We may view $X_{p}$ as an equivalence $\mathcal{K}_{X}(p, p)$-A-bimodule, in an obvious way. Also we may view $\mathcal{K}_{X}(p h, p)$ as a $C^{*}$-correspondence over $\mathcal{K}_{X}(p, p)$ with left action implemented by $\otimes 1_{h}$. With $\widetilde{X}_{p}$ denoting the dual correspondence, we clearly have isomorphisms of $C^{*}$-correspondences

$$
\mathcal{K}_{X}(p h, p) \cong X_{p h} \otimes_{A} \tilde{X}_{p} \cong X_{p} \otimes_{A} X_{h} \otimes_{A} \tilde{X}_{p}
$$

Hence $\mathcal{K}_{X}(p h, p)$ is an equivalence bimodule Morita equivalent to the equivalence $A$-bimodule $X_{h}$, cf. [22, Lemma 6.4]. Thus the assertion follows from [22, Corollary 6.3].

We recall that various criteria for amenability of ideals in right-tensor $C^{*}$-precategories are given in [19, Section 8]. For example, any such ideal is amenable when the underlying semigroup admits a controlled map into an amenable group, see [19, Theorem 8.4] in conjunction with the fact that any Fell bundle over an amenable group has amenable full sectional $C^{*}$-algebra.

Theorem 2.19 (Uniqueness Theorem for $\mathcal{N} \mathcal{T}(X)$ ). Let $X$ be a compactly-aligned product system over a right $L C M$ semigroup $P$ such that $\mathcal{K}_{X}$ is amenable. Suppose that either $P^{*}=\{e\}$ or that the Fell bundle $\left\{X_{h}\right\}_{h \in P^{*}}$ is aperiodic.

Consider the following conditions on a Nica covariant representation $\psi: X \rightarrow B(H)$ :

(i) $\psi$ satisfies condition $(C)$;

(ii) $\psi \rtimes P$ is an isomorphism from $\mathcal{N} \mathcal{T}(X)$ onto $\overline{\operatorname{span}}\left\{\psi(x) \psi(y)^{*}: x, y \in X\right\}$;

(iii) $\psi$ is injective and Toeplitz covariant.

Then (i) $\Rightarrow($ ii $) \Rightarrow$ (iii) and if $\phi_{p}(A) \subseteq \mathcal{K}\left(X_{p}\right)$ for every $p \in P$, then all these three conditions are equivalent. 
Proof. Taking into account Lemmas 2.4, 2.16 and Proposition 2.10, the assertion follows from [19, Corollary 10.14].

In general, condition (i) in Theorem 2.19 is stronger then (iii), cf. Example 2.22. It is an open problem whether, under the assumptions of Theorem 2.19, conditions (ii) and (iii) are always equivalent. We believe the answer to be affirmative and in the next result confirm this under an assumption of aperiodicity.

Proposition 2.20. Suppose that the LCM semigroup $P$ is a subsemigroup of a group $G$. Let $X$ be a compactly-aligned product system over $P$, and let $\mathcal{B}^{\theta}=\left\{B_{g}^{\theta}\right\}_{g \in G}$ be the Fell bundle associated to $\mathcal{K}_{X}$ and $\theta=\mathrm{id}$ in [19, Theorem 8.4]. If $\mathcal{B}^{\theta}$ is amenable and aperiodic, then for any Nica covariant representation $\psi: X \rightarrow B(H)$, the representation $\psi \rtimes P$ of $\mathcal{N} \mathcal{T}(X)$ is faithful if and only if $\psi$ is injective and Toeplitz covariant.

Proof. By [19, Proposition 12.10], see also [21, Corollary 4.3], $\psi \rtimes P$ is faithful on $\mathcal{N} \mathcal{T}(X)$ if and only if it is faithful on the core subalgebra $B_{e}^{\theta}=B_{e}^{X}$. By Theorem 2.17, this holds if and only if $\psi$ is injective and Toeplitz covariant. See also [19, Remark 10.13].

We can use condition (C) in its full force by exploiting the Doplicher-Roberts version of the Nica-Toeplitz algebra.

Theorem 2.21 (Uniqueness Theorem for $\mathcal{D} \mathcal{R}(\mathcal{N} \mathcal{T}(X))$ ). Let $X$ be a compactly-aligned product system over a right LCM semigroup $P$. Suppose that either $P^{*}=\{e\}$ or that the Fell bundle $\left\{X_{h}\right\}_{h \in P^{*}}$ is aperiodic. Assume also that $\mathcal{L}_{X}$ is amenable. Then for a Nica covariant representation $\psi: X \rightarrow B(H)$ the following are equivalent:

(i) $\psi$ satisfies condition $(C)$;

(ii) $\overline{\psi \rtimes P}$ is an isomorphism from $\mathcal{D} \mathcal{R}(\mathcal{N} \mathcal{T}(X))$ onto the closed linear span of operators $T$ satisfying $T \in \psi\left(X_{e}\right) \cup \psi\left(X_{e}\right)^{*}$ or

$T \in Q_{p}^{\psi} B(H) Q_{q}^{\psi}$ where $T \psi\left(X_{q}\right) \subseteq \psi\left(X_{p}\right)$ and $T^{*} \psi\left(X_{p}\right) \subseteq \psi\left(X_{q}\right)$, for $p, q \in P \backslash\{e\}$.

The isomorphism in item (ii) restricts, under the embedding $\mathcal{N} \mathcal{T}(X) \hookrightarrow \mathcal{D} \mathcal{R}(\mathcal{N} \mathcal{T}(X))$ ), to a natural isomorphism $\mathcal{N} \mathcal{T}(X) \cong \overline{\operatorname{span}}\left\{\psi(x) \psi(y)^{*}: x, y \in X\right\}$.

Proof. In view of Lemmas 2.4, 2.16, we may apply [19, Theorem 10.15]. To finish the proof, we need to show that for any $p, q \in P \backslash\{e\}$, we have

$$
\bar{\Psi}\left(\mathcal{L}\left(X_{q}, X_{p}\right)\right)=\left\{T \in Q_{p}^{\psi} B(H) Q_{q}^{\psi}: T \psi\left(X_{q}\right) \subseteq \psi\left(X_{p}\right) \text { and } T^{*} \psi\left(X_{p}\right) \subseteq \psi\left(X_{q}\right)\right\}
$$

where $\bar{\Psi}: \mathcal{L}_{X} \rightarrow B(H)$ is the extension of the Nica-Toeplitz representation $\Psi:=\left\{\Psi_{p, q}\right\}_{p, q \in P}$ of $\mathcal{K}_{X}$. This equality readily follows from the fact that $\psi$ is injective (and hence isometric on each fiber) and for $a \in \mathcal{L}\left(X_{q}, X_{p}\right), \bar{\Psi}(a)$ is determined by the formulas $\bar{\Psi}(a) \psi_{q}(x)=\psi_{p}(a x)$ and $\bar{\Psi}(a)\left(Q_{q}^{\psi}\right)^{\perp}=0$, where $x \in X_{q}$.

Example 2.22 (Doplicher-Roberts version of $\mathcal{O}_{\infty}$ ). We will now illustrate the above uniqueness results for $C^{*}$-algebras of product systems in the case of the Cuntz algebra $\mathcal{O}_{\infty}$. This in particular will explain the results and phenomena encountered in [13].

Let $\left\{u_{i}: i \in \mathbb{N}\right\} \subset \mathcal{B}(H)$ be a family of isometries with orthogonal ranges, so $u_{i}^{*} u_{j}=\delta_{i, j} 1$ for all $i, j \in \mathbb{N}$. For $n>0$ and any finite collection $i_{1}, \ldots, i_{n}$ of indices, let $u_{i_{1} i_{2} \ldots i_{n}}:=u_{i_{1}} u_{i_{2}} \ldots u_{i_{n}}$ and define

$$
X_{n}:=\overline{\operatorname{span}}\left\{u_{i_{1} i_{2} \ldots i_{n}}: i_{1}, \ldots, i_{n} \in \mathbb{N}\right\} \quad Q_{n}:=\sum_{i_{1}, \ldots, i_{n} \in \mathbb{N}} u_{i_{1} i_{2} \ldots i_{n}} u_{i_{1} i_{2} \ldots i_{n}}^{*} .
$$

We also put $X_{0}=\mathbb{C} I$ and $Q_{0}=1$. The family $X=\left\{X_{n}\right\}_{n \in \mathbb{N}}$ with operations inherited from $\mathcal{B}(H)$ becomes a product system over the semigroup $\mathbb{N}$ with coefficient algebra $A=\mathbb{C}$. For 
each $n>0, Q_{n}$ is the orthogonal projection onto the space $X_{n} H$. Note that (2.5), which is our geometric condition (C), is equivalent to asking that $Q_{1}$ is not equal to 1 , i.e.

$$
\sum_{i \in \mathbb{N}} u_{i} u_{i}^{*}<1
$$

where the infinite sum is defined using the strong operator topology. Since $\mathcal{N} \mathcal{T}(X)$ is generated by an infinite family of isometries with orthogonal ranges given by $\left\{i_{X}\left(u_{j}\right): j \in \mathbb{N}\right\},[4$, Theorem 1.12] gives an isomorphism

$$
\mathcal{N} \mathcal{T}(X) \cong \overline{\operatorname{span}}\left\{X_{n} X_{m}^{*}: n, m \in \mathbb{N}\right\} \cong \mathcal{O}_{\infty} .
$$

In particular, every countably infinite family of isometries with orthogonal ranges gives rise to an injective Nica-Toeplitz representation of $X$ - the algebraic condition (2.4) is satisfied automatically. We denote by $\mathcal{D} \mathcal{R}\left(\mathcal{O}_{\infty}\right)$ the Doplicher-Roberts algebra associated to $\left(X_{n}\right)_{n \in \mathbb{N}}$. Theorem 2.21 implies that (2.6) is equivalent to having an isomorphism

$$
\mathcal{D R}\left(\mathcal{O}_{\infty}\right) \cong \operatorname{span}\left\{\bigcup_{n, m \in \mathbb{N}}\left\{T \in Q_{m} B(H) Q_{n}: T X_{n} \subseteq X_{m} \text { and } T^{*} X_{m} \subseteq X_{n}\right\}\right\}
$$

Without condition (2.6), all we can say is that there is a surjective homomorphism from $\mathcal{D} \mathcal{R}\left(\mathcal{O}_{\infty}\right)$ to the right-hand side of (2.8) obtained from the universal property of $\mathcal{D} \mathcal{R}(\mathcal{N} \mathcal{T}(X))$.

This example illustrates the fact that condition (C) captures uniqueness of the DoplicherRoberts algebra $\mathcal{D} \mathcal{R}\left(\mathcal{O}_{\infty}\right)$, which is a $C^{*}$-algebra containing $\mathcal{O}_{\infty}$, and that uniqueness of $\mathcal{O}_{\infty}$ as a $C^{*}$-algebra generated by isometries with orthogonal ranges is independent of condition (C). This phenomenon is consistent with our Theorem 2.19, as the left action of $A=\mathbb{C} 1$ on $X_{1} \cong \ell^{2}(\mathbb{N})$ is not by generalized compacts.

In order to get an efficient uniqueness theorem for $\mathcal{O}_{\infty}$ one needs to view it as a NicaToeplitz algebra over the free semigroup $\mathbb{F}_{\mathbb{N}}^{+}$. This idea, in disguise, was exploited in [13]. With our results in hand we can make it formal and explicit. Note that any product system over a free semigroup $\mathbb{F}_{\Lambda}^{+}$is automatically compactly-aligned.

Lemma 2.23. Let $Y:=\bigoplus_{\lambda \in \Lambda} Y_{\lambda}$ be a direct sum of $C^{*}$-correspondences $Y_{\lambda}, \lambda \in \Lambda$, over a $C^{*}$-algebra $A$. There is a product system $X=\left\{X_{p}\right\}_{p \in \mathbb{F}_{\Lambda}^{+}}$over $A$ such that for any word $p=\lambda_{1} \ldots \lambda_{n} \in \mathbb{F}_{\Lambda}^{+}$we have

$$
X_{p}:=Y_{\lambda_{1}} \otimes Y_{\lambda_{2}} \otimes \cdots \otimes Y_{\lambda_{n}}
$$

and the product in $X$ is given by the iterated internal tensor product. We have a one-to-one correspondence between Nica covariant representations $\Psi$ of $X=\left\{X_{p}\right\}_{p \in \mathbb{F}_{\Lambda}^{+}}$and representations $(\pi, \psi)$ of the $C^{*}$-correspondence $Y$ where

$$
\Psi\left(y_{\lambda_{1}} \otimes y_{\lambda_{2}} \otimes \cdots \otimes y_{\lambda_{n}}\right)=\psi\left(y_{\lambda_{1}}\right) \psi\left(y_{\lambda_{2}}\right) \ldots \psi\left(y_{\lambda_{n}}\right), \quad y_{\lambda_{i}} \in Y_{\lambda_{i}}, i=1, \ldots, n .
$$

Thus we have a natural isomorphism $\mathcal{T}_{Y} \cong \mathcal{N} \mathcal{T}(X)$.

Proof. The proof is straightforward. We leave the details to the reader.

Corollary 2.24. Let $Y=\bigoplus_{\lambda \in \Lambda} Y_{\lambda}$ be a direct sum of $C^{*}$-correspondences $Y_{\lambda}, \lambda \in \Lambda$, over a $C^{*}$-algebra $A$. Consider the following conditions that a representation $(\pi, \psi)$ of the $C^{*}$ correspondence $Y$ in a Hilbert space $H$ may satisfy:

(i) A acts, via $\pi$, faithfully on $\left(\psi\left(\bigoplus_{\lambda \in F} Y_{\lambda}\right) H\right)^{\perp}$ for every finite subset $F$ of $\Lambda$;

(ii) The $C^{*}$-algebra generated by $\pi(A) \cup \psi(Y)$ is naturally isomorphic to $\mathcal{T}_{Y}$;

(iii) $\pi(A) \cap \overline{\operatorname{span}}\left\{\psi(x) \psi(y)^{*}: x, y \in Y_{\lambda}, \lambda \in F\right\}=\{0\}$ for every finite $F \subseteq \Lambda$. 
Then $(i) \Rightarrow($ ii $) \Rightarrow$ (iii). Moreover, if $A$ acts by generalized compacts on the left of each $Y_{\lambda}$, $\lambda \in \Lambda$, then all the above conditions are equivalent.

Proof. Let $X=\left\{X_{p}\right\}_{p \in \mathbb{F}_{\Lambda}^{+}}$be the product system described in Lemma 2.23. Since $\mathcal{N} \mathcal{T}(X)$ and $\mathcal{N} \mathcal{T}^{r}(X)$ are isomorphic by $\left[19\right.$, Corollary 8.6] and $\left(\mathbb{F}_{\Lambda}^{+}\right)^{*}=\{e\}$, we may apply Theorem 2.19 to $X$. Translating the result, using Lemma 2.23 , to $C^{*}$-correspondences $Y_{\lambda}, \lambda \in \Lambda$, we get the assertions.

Remark 2.25. The relationship between conditions (i) and (ii) in Corollary 2.24 was established in [13, Theorem 3.1]. The algebraic condition (iii), which is what we call Toeplitz covariance, in general does not imply (i), see Example 2.22. We have already seen pieces of evidence that in general Toeplitz covariance could be the right condition for characterizing uniqueness of $\mathcal{N} \mathcal{T}(X)$. Another evidence for this is again the case of $\mathcal{O}_{\infty}$, as we shall now explain.

If we specialize Corollary 2.24 to the $C^{*}$-correspondence $X_{1} \cong \ell^{2}(\mathbb{N})$ over $\mathbb{C}$ from Example 2.22, then we may view $X_{1}$ as a direct sum over $\mathbb{N}$ of finite dimensional (even one dimensional) spaces $Y_{n}$. It is readily seen that for every representation of $X$ coming from a countably infinite family of isometries with orthogonal ranges, both of conditions (i) and (iii) in Corollary 2.24 are satisfied. Since the left action is by compacts in each $Y_{n}, n \geq 1$, we may use each of these conditions to recover the uniqueness of $\mathcal{O}_{\infty}$.

2.4. Semigroup $C^{*}$-algebras twisted by product systems. Let $X$ be a compactlyaligned product system over a right LCM semigroup $P$. For each $p \in P$, let $\mathbb{1}_{p} \in \ell^{\infty}(P)$ be the characteristic function of $p P$. Since the product $\mathbb{1}_{p} \mathbb{1}_{q}$ is either $\mathbb{1}_{r}$ (if $p P \cap q P=r P$ ) or 0 , we have that $B_{P}:=\overline{\operatorname{span}}\left\{\mathbb{1}_{p}: p \in P\right\}$ is a $C^{*}$-subalgebra of $\ell^{\infty}(P)$. Moreover, the projections $\mathbb{1}_{p}$ form a semilattice isomorphic to $J(P)$. Recall that $1_{r}$ denotes the identity in $\mathcal{L}\left(X_{r}\right)$ for every $r \in P$. If 1 is the identity in the unitization $\mathcal{D} \mathcal{R}(\mathcal{N} \mathcal{T}(X))^{\sim}$ of $\mathcal{D} \mathcal{R}(\mathcal{N} \mathcal{T}(X))$, then the projections $\left\{i_{\mathcal{L}_{X}}\left(1_{p}\right)\right\}_{p \in P \backslash\{e\}} \cup\{1\}$ form a semilattice isomorphic to $J(P)$, cf. [19, Lemma 5.8]. Since the family $J(P)$ is independent, see [1, Corollary 3.6], it follows from [28, Proposition 2.4] that the assignment

$$
B_{P} \ni \mathbb{1}_{p} \longmapsto i_{\mathcal{L}_{X}}\left(1_{p}\right) \in \mathcal{D} \mathcal{R}(\mathcal{N} \mathcal{T}(X)), \quad p \in P \backslash\{e\},
$$

extends uniquely to an injective unital homomorphism $B_{P} \hookrightarrow \mathcal{D} \mathcal{R}(\mathcal{N} \mathcal{T}(X))^{\sim}$. We will use it to identify $B_{P}$ with a $C^{*}$-subalgebra of $\mathcal{D} \mathcal{R}(\mathcal{N} \mathcal{T}(X))^{\sim}$.

Definition 2.26. Let $X$ be a compactly-aligned product system. We call the $C^{*}$-algebra

$$
\mathcal{F} \mathcal{R}(X):=C^{*}\left(B_{P} \cdot \mathcal{N} \mathcal{T}(X)\right) \subseteq \mathcal{D} \mathcal{R}(\mathcal{N} \mathcal{T}(X)),
$$

generated by elements $i_{\mathcal{L}_{X}}\left(1_{p}\right) i_{\mathcal{K}_{X}}(a)$ where $a \in \mathcal{K}(q, r), p, q, r \in P, p \neq e$, the Fowler-Raeburn algebra of $X$ or the semigroup $C^{*}$-algebra of $P$ twisted by $X$.

Proposition 2.27. We have $\mathcal{F} \mathcal{R}(X)=\overline{\operatorname{span}}\left\{i_{\mathcal{K}_{X}}(x) i_{\mathcal{L}_{X}}\left(1_{p}\right) i_{\mathcal{K}_{X}}(y)^{*}: x, y \in X, p \in P\right\}$. In particular, $B_{P} \subseteq M(\mathcal{F} \mathcal{R}(X))$. Moreover, $\mathcal{F} \mathcal{R}(X)=\mathcal{N} \mathcal{T}(X)$ if and only if the left action of $A$ on each fiber $X_{p}$ is by generalized compacts.

Proof. For any $x \in X$ and $p \in P$, using Nica covariance of $i_{\mathcal{L}_{X}}$ twice, we get $i_{\mathcal{K}_{X}}(x) i_{\mathcal{L}_{X}}\left(1_{p}\right)=$ $i_{\mathcal{L}_{X}}\left(x \otimes 1_{p}\right)=i_{\mathcal{L}_{X}}\left(1_{d(x) p}\right) i_{\mathcal{K}_{X}}(x)$, and similarly

$$
i_{\mathcal{L}_{X}}\left(1_{p}\right) i_{\mathcal{K}_{X}}(x)= \begin{cases}i_{\mathcal{K}_{X}}(x) i_{\mathcal{L}_{X}}\left(1_{d(x)^{-1} r}\right) & \text { if } p P \cap d(x) P=r P, \\ 0, & \text { otherwise. }\end{cases}
$$

This implies that $B_{P} \cdot \mathcal{N} \mathcal{T}(X) \subseteq \overline{\operatorname{span}}\left\{i_{\mathcal{K}_{X}}(x) i_{\mathcal{L}_{X}}\left(1_{p}\right) i_{\mathcal{K}_{X}}(y)^{*}: x, y \in X, p \in P\right\} \subseteq \mathcal{F} \mathcal{R}(X)$. Hence to prove the first part of the assertion, it suffices to show that the product of two 
elements of the form $i_{\mathcal{K}_{X}}(x) i_{\mathcal{L}_{X}}\left(1_{p}\right) i_{\mathcal{K}_{X}}(y)^{*}$ and $i_{\mathcal{K}_{X}}(z) i_{\mathcal{L}_{X}}\left(1_{s}\right) i_{\mathcal{K}_{X}}(w)^{*}, x, y, z, w \in X, p, s \in$ $P$, can be approximated by a finite sum of elements of that form. The product $i_{\mathcal{K}_{X}}(y)^{*} i_{\mathcal{K}_{X}}(z)$ can be approximated by a finite sum of elements of the form $i_{\mathcal{K}_{X}}(f) i_{\mathcal{K}_{X}}(g)^{*}, f, g \in X$. Applying (2.9) twice, we see that the product

$$
i_{\mathcal{K}_{X}}(x) i_{\mathcal{L}_{X}}\left(1_{p}\right) i_{\mathcal{K}_{X}}(f) i_{\mathcal{K}_{X}}(g)^{*} i_{\mathcal{L}_{X}}\left(1_{s}\right) i_{\mathcal{K}_{X}}(w)^{*}
$$

is either zero or of the form $i_{\mathcal{K}_{X}}(x f) i_{\mathcal{L}_{X}}\left(1_{t}\right) i_{\mathcal{K}_{X}}(w g)^{*}$. Thus $\mathcal{F} \mathcal{R}(X)$ is the closed linear span of $\left\{i_{\mathcal{K}_{X}}(x) i_{\mathcal{L}_{X}}\left(1_{p}\right) i_{\mathcal{K}_{X}}(y)^{*}: x, y \in X, p \in P\right\}$. Now, (2.9) implies $B_{P} \subseteq M(\mathcal{F} \mathcal{R}(X))$.

If the left action of $A$ on each fiber $X_{p}$ is by compact operators, then $\mathcal{K}_{X} \otimes 1 \subseteq \mathcal{K}_{X}$, by Lemma 2.4. Hence $i_{\mathcal{K}_{X}}(x) i_{\mathcal{L}_{X}}\left(1_{p}\right) i_{\mathcal{K}_{X}}(y)^{*}=i_{\mathcal{K}_{X}}\left(x \otimes 1_{p}\right) i_{\mathcal{K}_{X}}(y)^{*} \in \mathcal{N} \mathcal{T}(X)$ for every $x, y \in X$ and $p \in P$. Therefore $\mathcal{F} \mathcal{R}(X) \subseteq \mathcal{N} \mathcal{T}(X)$ and the reverse inclusion is obvious.

Conversely, if $\mathcal{F} \mathcal{R}(X) \subseteq \mathcal{N} \mathcal{T}(X)$, then for any $a \in A$ and $p \in P \backslash\{e\}$ we have that $i_{\mathcal{L}_{X}}\left(\phi_{p}(a)\right)=i_{\mathcal{L}_{X}}\left(1_{p}\right) i_{\mathcal{K}_{X}}(a) \in \mathcal{N} \mathcal{T}(X)$. Hence for any $\varepsilon>0$ there is a finite sum of the form $S=\sum_{s, t} i_{\mathcal{K}_{X}}\left(a_{s, t}\right)$ where $a_{s, t} \in \mathcal{K}\left(X_{t}, X_{s}\right)$ such that $\left\|i_{\mathcal{L}_{X}}\left(\phi_{p}(a)\right)-S\right\|<\varepsilon$. Hence $\| E^{\mathbb{L}}\left(\Lambda\left(i_{\mathcal{L}_{X}}\left(\phi_{p}(a)\right)\right)-E^{\mathbb{L}}(\Lambda(S)) \|<\varepsilon\right.$ where $E^{\mathbb{L}}$ is the transcendental conditional expectation on $\mathcal{N} \mathcal{T}^{r}(X)$ constructed in [19]. By [19, Proposition 5.4], cf. also [19, Remark 5.7], we have

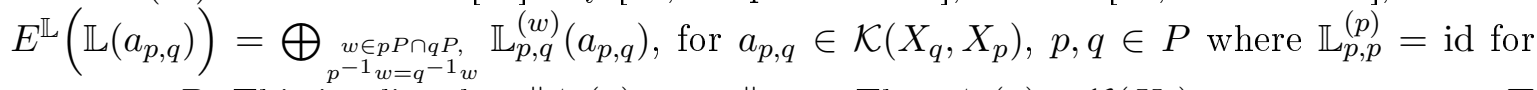
every $p \in P$. This implies that $\left\|\phi_{p}(a)-a_{p, p}\right\|<\varepsilon$. Thus $\phi_{p}(a) \in \mathcal{K}\left(X_{p}\right)$.

Left translation on $\ell^{\infty}(P)$ restricts to a unital semigroup homomorphism $\tau: P \rightarrow \operatorname{End}\left(\psi_{e}(A)^{\prime}\right)$, determined by $\tau_{q}\left(\mathbb{1}_{p}\right)=\mathbb{1}_{q p}$ for $p, q \in P$. The isometric crossed product $B_{P} \rtimes_{\tau} P$ is naturally isomorphic to the semigroup $C^{*}$-algebra $C^{*}(P)$, see [27, Lemma 2.14], so $\mathcal{F} \mathcal{R}(X)$ may be viewed as a version of $C^{*}(P)$ twisted by $X$, see [12], [11]. We make this explicit in our setting.

Lemma 2.28. Let $\psi$ be a nondegenerate representation of $X$ on a Hilbert space $H$. For each $p \in P \backslash\{e\}$ there is a unique endomorphism $\alpha_{p}^{\psi}$ of $\psi_{e}(A)^{\prime}$ such that

$$
\alpha_{p}^{\psi}(S) \psi_{p}(x)=\psi_{p}(x) S, \quad \text { for all } S \in \psi_{e}(A)^{\prime}, x \in X_{p},
$$

and $\alpha_{p}^{\psi}(1)$ vanishes on $\left(\psi_{p}\left(X_{p}\right) H\right)^{\perp}$. We put $\alpha_{e}^{\psi}=\mathrm{id}$. Then $\alpha^{\psi}: P \rightarrow \operatorname{End}\left(\psi_{e}(A)^{\prime}\right)$ is a unital semigroup homomorphism.

Proof. The existence of $\alpha_{p}^{\psi}$ for each $p \in P$ is proved in [11, Proposition 4.1 (1)]. The semigroup law $\alpha_{p}^{\psi} \circ \alpha_{q}^{\psi}=\alpha_{p q}^{\psi}$ for $p, q \in P \backslash\{e\}$ is proved in [11, Proposition 4.1 (2)]. To allow $p=e$, Fowler assumes all $X_{p}$ are essential. With our definition of $\alpha_{e}^{\psi}$, the semigroup law follows if one or both of $p, q$ equal $e$ by a direct verification.

Definition 2.29. A covariant representation of the quadruple $\left(B_{P}, P, \tau, X\right)$ on a Hilbert space $H$ is a pair $(\pi, \psi)$ consisting of a nondegenerate representation $\pi: B_{P} \rightarrow B(H)$ and a nondegenerate representation $\psi: X \rightarrow B(H)$ such that $\pi\left(B_{P}\right) \subseteq \psi_{e}(A)^{\prime}$ and $\pi \circ \tau_{p}=\alpha_{p}^{\psi} \circ \pi$, $p \in P$, where $\alpha^{\psi}: P \rightarrow \operatorname{End}\left(\psi_{e}(A)^{\prime}\right)$ is defined in Lemma 2.28.

Proposition 2.30. There is a bijective correspondence between covariant representations $(\pi, \psi)$ of $\left(B_{P}, P, \tau, X\right)$ and Nica covariant representations $\psi$ of $X$ implemented by $\pi\left(\mathbb{1}_{p}\right)=$ $\alpha_{p}^{\psi}(1)$ for $p \in P$.

In particular, there is a covariant representation $\left(i_{B_{P}}, i_{X}\right)$ of $\left(B_{P}, P, \tau, X\right)$ such that

1) $\mathcal{F} \mathcal{R}(X)=C^{*}\left(i_{B_{P}}\left(B_{P}\right) i_{X}(X)\right)$

2) for every covariant representation $(\pi, \psi)$ of $\left(B_{P}, P, \tau, X\right)$ there is a representation $\pi \rtimes \psi$ of $\mathcal{F} \mathcal{R}(X)$ such that $\overline{\pi \rtimes \psi} \circ i_{B_{P}}=\pi$ and $\overline{\pi \rtimes \psi} \circ i_{X}=\psi$. 
Proof. If $(\pi, \psi)$ is a covariant representation of $\left(B_{P}, P, \tau, X\right)$, then $\pi\left(\mathbb{1}_{p}\right)=\alpha_{p}^{\psi}(1), p \in P$ and this relation determines $\pi$. Moreover, we have $\pi\left(\mathbb{1}_{p}\right)=\alpha_{p}^{\psi}(1)=Q_{p}^{\psi}$, and therefore $\psi$ is Nica covariant by Lemma 2.14. Conversely, if $\psi$ is a Nica covariant representations of $X$, then $\alpha_{p}^{\psi}(1)=Q_{p}^{\psi}$ satisfy $(2.2)$ and belong to $\psi_{e}(A)^{\prime}$, cf. [19, Proposition 9.5]. Hence there is a representation $\pi: B_{P} \rightarrow \psi_{e}(A)^{\prime}$ determined by $\pi\left(\mathbb{1}_{p}\right)=\alpha_{p}^{\psi}(1), p \in P$, by [28, Proposition 2.4]. Since $\pi\left(\tau_{p}\left(\mathbb{1}_{q}\right)\right)=\pi\left(\mathbb{1}_{p q}\right)=\alpha_{p q}^{\psi}(1)=\alpha_{p}^{\psi}\left(\alpha_{q}^{\psi}(1)\right)=\alpha_{p}^{\psi}\left(\pi\left(\mathbb{1}_{q}\right)\right)$ for every $p, q \in P$, we conclude that $(\pi, \psi)$ is a covariant representation of $\left(B_{P}, P, \tau, X\right)$.

The second part of the assertion is now immediate (by representing $\mathcal{F} \mathcal{R}(X)$ faithfully and nondegenerately on a Hilbert space).

Theorem 2.31 (Uniqueness Theorem for $\mathcal{F} \mathcal{R}(X)$ ). Retaining the assumptions in Theorem 2.21 each of the conditions (i) and (ii) therein are equivalent to the following one:

(iii) $\mathcal{F} \mathcal{R}(X) \cong \overline{\operatorname{span}}\left\{\psi(x) Q_{p}^{\psi} \psi(y)^{*}: x, y \in X, p \in P\right\}$. In particular, $\pi \rtimes \psi$ is faithful.

Proof. The implication (ii) $\Rightarrow$ (iii) is obvious as $\mathcal{F} \mathcal{R}(X) \subseteq \mathcal{D} \mathcal{R}(\mathcal{N} \mathcal{T}(X))$. To see that (iii) $\Rightarrow$ (i) follows because condition (2.5) involves only elements lying in the image of the corresponding representation of $\mathcal{F} \mathcal{R}(X)$. Hence if they are satisfied in $\mathcal{D} \mathcal{R}(\mathcal{N} \mathcal{T}(X))$ they need to be satisfied in $\mathcal{F} \mathcal{R}(X)$.

Remark 2.32. When $X$ is compactly aligned, $P$ is a positive cone in a quasi-lattice ordered group and all the fibers $X_{p}, p \in P$, are essential, then $\mathcal{F} \mathcal{R}(X)$ coincides with the algebra denoted by $B_{P} \rtimes_{\tau, X} P$ in [11], see also [12]. In this case the equivalence of (i) and (iii) in Theorem 2.31 is [11, Theorem 7.2], which in turn is a generalization of [12, Theorem 5.1].

Example 2.33 (Fowler-Raeburn version of $\mathcal{O}_{\infty}$ ). Retain the notation of Example 2.22. We noticed there that conditions (2.6) and (2.8) are equivalent. Denoting by $\mathcal{F} \mathcal{R}\left(\mathcal{O}_{\infty}\right)$ the FowlerRaeburn algebra $\mathcal{F} \mathcal{R}(X)$ associated to $\left(X_{n}\right)_{n \in \mathbb{N}}$ we see now, using Theorem 2.31 , that these equivalent conditions are further equivalent to having an isomorphism

$$
\mathcal{F} \mathcal{R}\left(\mathcal{O}_{\infty}\right) \cong \overline{\operatorname{span}}\left\{X_{n} Q_{k} X_{m}^{*}: n, m, k \in \mathbb{N}\right\} .
$$

In particular, $\mathcal{O}_{\infty}$ embeds as a proper subalgebra of $\mathcal{F} \mathcal{R}\left(\mathcal{O}_{\infty}\right)$ by $(2.7)$ and the second part of Proposition 2.27, cf. [12, Example 5.6(2)]. The algebra $\mathcal{F} \mathcal{R}\left(\mathcal{O}_{\infty}\right)$ is separable while $\mathcal{D} \mathcal{R}\left(\mathcal{O}_{\infty}\right)$ is not.

\section{Nica-Toeplitz Crossed products By COMPletely Positive maps}

In this section we will introduce a general definition of Nica-Toeplitz $C^{*}$-algebra for an action of a LCM semigroup by completely positive maps. We will do it in two steps. First we introduce a Toeplitz $C^{*}$-algebra, and then obtain a Nica-Toeplitz $C^{*}$-algebra as a quotient by 'eliminating redundancies'. In the next sections we will analyze these $C^{*}$-algebras in more detail in two special cases. Namely, when the action is by endomorphisms or by transfer operators.

3.1. General construction. Let $P$ be a right LCM semigroup. Let $\mathrm{CP}(A)$ denote a semigroup of completely positive maps on a $C^{*}$-algebra $A$ (with semigroup operation given by composition).

Definition 3.1. Let $\varrho: P \ni p \mapsto \varrho_{p} \in \mathrm{CP}(A)$ be a unital semigroup antihomomorphism, i.e. $\alpha_{e}=i d$ and $\varrho_{q} \circ \varrho_{p}=\varrho_{p q}$ for all $p, q \in P$. We call $(A, P, \varrho)$ a $C^{*}$-dynamical system.

A representation of the semigroup $P$ in a Hilbert space $H$ is a unital semigroup homomorphism $S: P \rightarrow \mathcal{B}(H)$ into the multiplicative semigroup of $\mathcal{B}(H)$. The following is an obvious semigroup generalization of [18, Definition 3.1]. 
Definition 3.2. A representation of a $C^{*}$-dynamical system $(A, P, \varrho)$ on a Hilbert space $H$ is a pair $(\pi, S)$ consisting of a nondegenerate representation $\pi: A \rightarrow \mathcal{B}(H)$ and a homomorphism $S: P \rightarrow \mathcal{B}(H)$ such that

$$
S_{p}^{*} \pi(a) S_{p}=\pi\left(\varrho_{p}(a)\right)
$$

for all $p, q \in P$ and $a \in A$. We put $C^{*}(\pi, S):=C^{*}\left(\bigcup_{p \in P} \pi(A) S_{p}\right)$. Exactly as in the proof of [18, Lemma 3.2], one can prove that there is a universal representation $\left(i_{A}, \hat{t}\right)$ of $(A, P, \varrho)$; universal in the sense that for any other representation $(\pi, S)$ of $(A, P, \varrho)$ the maps

$$
i_{A}(a) \longmapsto \pi(a), \quad i_{A}(a) \hat{t}_{p} \longmapsto \pi(a) S_{p}, \quad a \in A, p \in P,
$$

give rise to an epimorphism from $C^{*}\left(i_{A}, \hat{t}\right)$ onto $C^{*}(\pi, S)$. Up to a natural isomorphism the $C^{*}$-algebra $\mathcal{T}(A, P, \varrho):=C^{*}\left(i_{A}(A), \hat{t}\right)$ is uniquely determined by $(A, P, \varrho)$, and we call it the Toeplitz algebra of $(A, P, \varrho)$.

For any $C^{*}$-algebra $C$ we denote by $\mathcal{R} \mathcal{M}(C), \mathcal{L} \mathcal{M}(C)$ and $\mathcal{M}(C)$ the algebras of right, left and two-sided multipliers of $C$, respectively, cf. [31,3.12]. We say that a map $\varrho$ on $C$ is strict if for any approximate unit $\left\{\mu_{\lambda}\right\}$ in $C$, the net $\left\{\varrho\left(\mu_{\lambda}\right)\right\}$ converges strictly to a multiplier of $C$.

Lemma 3.3. We have $\mathcal{T}(A, P, \varrho)=C^{*}\left(\bigcup_{p \in P} i_{A}(A) \hat{t}_{p} i_{A}(A)\right)$. Hence $i_{A}(A)$ is a nondegenerate subalgebra of $\mathcal{T}(A, P, \varrho)$ and $\left\{\hat{t}_{p}\right\}_{p \in P} \subseteq \mathcal{R} \mathcal{M}(\mathcal{T}(A, P, \varrho))$. If every $\varrho_{p}, p \in P$, is strict then $\mathcal{T}(A, P, \varrho)=C^{*}\left(\bigcup_{p \in P} \hat{t}_{p} i_{A}(A)\right)$ and $\left\{\hat{t}_{p}\right\}_{p \in P} \subseteq \mathcal{M}(\mathcal{T}(A, P, \varrho))$.

Proof. Suppose that $\mathcal{T}(A, P, \varrho)$ acts in a nondegenerate way on a Hilbert space $H$. By [18, Proposition 3.10 and Lemma 3.8], for any $p \in P$ and an approximate unit $\left\{\mu_{\lambda}\right\}_{\lambda \in \Lambda}$ in $A$ we have s- $\lim _{\lambda \in \Lambda} i_{A}\left(\mu_{\lambda}\right) \hat{t}_{p}=\hat{t}_{p}$ and $i_{A}(A) \hat{t}_{p} \subseteq i_{A}(A) \hat{t}_{p} i_{A}(A)$. In particular, $\hat{t}_{p} \in \mathcal{T}(A, P, \varrho)^{\prime \prime}$ and $\mathcal{T}(A, P, \varrho) \subseteq C^{*}\left(\bigcup_{p \in P} i_{A}(A) \hat{t}_{p} i_{A}(A)\right)$. The reverse inclusion $C^{*}\left(\bigcup_{p \in P} i_{A}(A) \hat{t}_{p} i_{A}(A)\right) \subseteq$ $\mathcal{T}(A, P, \varrho)$ is clear since $i_{A}(A)=i_{A}(A) \hat{t}_{e} \subseteq \mathcal{T}(A, P, \varrho)$. Thus $i_{A}(A)$ is a nondegenerate subalgebra of $\mathcal{T}(A, P, \varrho)$. Every $b \in \mathcal{T}(A, P, \varrho)$ is of the form $b^{\prime} i_{A}(a)$, where $b^{\prime} \in \mathcal{T}(A, P, \varrho)$, $a \in A$, and $b \hat{t}_{p}=b^{\prime} i_{A}(a) \hat{t}_{p} \in b^{\prime} i_{A}(A) \hat{t}_{p} i_{A}(A) \subseteq \mathcal{T}(A, P, \varrho)$. Hence $\hat{t}_{p} \in \mathcal{R} \mathcal{M}(\mathcal{T}(A, P, \varrho))$, for every $p \in P$.

Suppose now that every map $\varrho_{p}, p \in P$, is strict. By [18, Proposition 3.10 and Remark 3.9], we get $\hat{t}_{p} i_{A}(A) \subseteq i_{A}(A) \hat{t}_{p} i_{A}(A)$, for every $p \in P$. Using this, similarly as above, one gets that $\mathcal{T}(A, P, \varrho)=C^{*}\left(\bigcup_{p \in P} \hat{t}_{p} i_{A}(A)\right)$ and $\hat{t}_{p} \in \mathcal{L} \mathcal{M}(\mathcal{T}(A, P, \varrho))$, for every $p \in P$.

Let $(\pi, S)$ be a representation of $(A, P, \varrho)$. In view of $(3.1)$ the Banach spaces

$$
\mathcal{K}_{(\pi, S)}(p, q):=\overline{\pi(A) S_{p} \pi(A) S_{q}^{*} \pi(A)}, \quad p, q \in P,
$$

form a $C^{*}$-precategory. In general, it is not obvious that there exists a right-tensor $C^{*}$ precategory containing $\mathcal{K}_{(\pi, S)}$ as an ideal. Nevertheless, we can mimic the definition of Nica covariance to define a Nica-Toeplitz algebra as follows, where we also draw on inspiration from [6].

Definition 3.4. Let $(\pi, S)$ be a representation of $(A, P, \varrho)$. We say that a pair $(a \cdot b, k)$ is a redundancy for $(\pi, S)$ if $a \in \mathcal{K}_{(\pi, S)}(p, q), b \in \mathcal{K}_{(\pi, S)}(s, t)$ and $k \in \mathcal{K}_{(\pi, S)}\left(p q^{-1} r, t s^{-1} r\right)$, for some $p, q, s, t, r \in P$ with $q P \cap s P=r P$, are such that

$$
a b \pi(c) S_{t s^{-1} r}=k \pi(c) S_{t s^{-1} r} \text { for all } c \in A .
$$

We say that $(\pi, S)$ is Nica covariant if

(1) for every redundancy $(a \cdot b, k)$ we have $a \cdot b=k$;

(2) $\mathcal{K}_{(\pi, S)}(p, q) \mathcal{K}_{(\pi, S)}(s, t)=\{0\}$ whenever $q P \cap s P=\emptyset$. 
Remark 3.5. Note that if $(a \cdot b, k)$ is a redundancy for $(\pi, S)$, then $a \cdot b$ determines $k$ uniquely, via (3.3). Indeed, just note that the essential subspace for $k$ is

$$
\overline{\mathcal{K}_{(\pi, S)}\left(t s^{-1} r, t s^{-1} r\right) H}=\overline{\pi(A) S_{t s^{-1} r} \pi(A) H}=\overline{\pi(A) S_{t s^{-1} r} H} .
$$

We define the Nica-Toeplitz algebra of the $C^{*}$-dynamical system $(A, P, \varrho)$ to be the $C^{*}$ algebra $\mathcal{N} \mathcal{T}(A, P, \varrho):=C^{*}\left(j_{A}, \hat{s}\right)$ generated by a universal Nica covariant representation $\left(j_{A}, \hat{s}\right)$ of $(A, P, \varrho)$. As the next result shows, there is an alternate way to justify its existence.

Lemma 3.6. We have $\mathcal{N} \mathcal{T}(A, P, \varrho) \cong \mathcal{T}(A, P, \varrho) / \mathcal{N}$ where $\mathcal{N}$ is the ideal of $\mathcal{T}(A, P, \varrho)$ generated by the differences

$$
a \cdot b-k \quad \text { where }(a \cdot b, k) \text { is a redundancy for }\left(i_{A}, \hat{t}\right)
$$

and products

$$
a \cdot b \quad \text { where } a \in \mathcal{K}_{\left(i_{A}, \hat{t}\right)}(p, q), b \in \mathcal{K}_{\left(i_{A}, \hat{t}\right)}(s, t) \text { and } q P \cap s P=\emptyset .
$$

Proof. It is straightforward and therefore left to the reader.

We aim to investigate uniqueness of representations of $\mathcal{N} \mathcal{T}(A, P, \rho)$. We will do this by specializing to two classes of actions where $\mathcal{N} \mathcal{T}(A, P, \rho)$ admits realizations as a Nica-Toeplitz algebra of a right-tensor $C^{*}$-precategory.

Remark 3.7. Even though in the greatest generality of an action by completely positive maps there is no obvious structure of a right-tensor category, it is still possible to define a notion similar to well-aligned for $C^{*}$-precategory and show that it provides a structural description of $\mathcal{N} \mathcal{T}(A, P, \rho)$ similar to the Nica-Toeplitz algebra of a $C^{*}$-precategory, cf. [19, Remark 3.9]. We include the details here for two reasons: first of all because the classes of examples we consider exhibit this additional feature and second because we believe the observation may be of use in future investigations.

We say that a $C^{*}$-dynamical system $(A, P, \varrho)$ is well-aligned if for every representation $(\pi, S)$ of $(A, P, \varrho)$ and all pairs $a \in \mathcal{K}_{(\pi, S)}(p, q)$ and $b \in \mathcal{K}_{(\pi, S)}(s, t)$ with $q P \cap s P=r P$ there is $k \in \mathcal{K}_{(\pi, S)}\left(p q^{-1} r, t s^{-1} r\right)$ such that $(a \cdot b, k)$ is a redundancy for $(\pi, S)$ (obviously it suffices to check this requirement only for the universal representation $\left.\left(i_{A}, \hat{t}\right)\right)$.

We now claim that if a $C^{*}$-dynamical system $(A, P, \varrho)$ is well-aligned, then

$$
\mathcal{N} \mathcal{T}(A, P, \varrho)=\overline{\operatorname{span}}\left\{\bigcup_{p, q \in P} \mathcal{K}_{\left(j_{A}, \hat{s}\right)}(p, q)\right\} .
$$

Indeed, the Banach space $\overline{\operatorname{span}}\left\{\bigcup_{p, q \in P} \mathcal{K}_{\left(j_{A}, \hat{s}\right)}(p, q)\right\}$ is closed under taking adjoints. Thus we only need to check that it is closed under multiplication. Let $a \in \mathcal{K}_{\left(j_{A}, \hat{s}\right)}(p, q)$ and $b \in$ $\mathcal{K}_{\left(j_{A}, \hat{s}\right)}(s, t)$. If $q P \cap s P=\emptyset$, then $a \cdot b=0$ by Nica covariance of $\left(j_{A}, \hat{s}\right)$. Assume then that $q P \cap s P=r P$. By well-alignment there is $k \in \mathcal{K}_{\left(j_{A}, \hat{s}\right)}\left(p q^{-1} r, t s^{-1} r\right)$ such that $(a \cdot b, k)$ is a redundancy for $\left(j_{A}, \hat{s}\right)$. Hence $a \cdot b=k \in \mathcal{K}_{\left(j_{A}, \hat{s}\right)}\left(p q^{-1} r, t s^{-1} r\right)$, again by Nica covariance.

3.2. Nica-Toeplitz crossed products by endomorphisms. Throughout this subsection we let $P$ be a right LCM semigroup and denote by $\alpha: P \ni p \mapsto \alpha_{p} \in \operatorname{End}(A)$ a unital semigroup antihomomorphism, i.e. $\alpha_{e}=i d$ and $\alpha_{q} \circ \alpha_{p}=\alpha_{p q}$ for all $p, q \in P$, where we assume that $\alpha_{p}$ is an endomorphism of $A$ for each $p \in P$. Since $*$-homomorphisms are completely positive maps, $(A, P, \alpha)$ is a $C^{*}$-dynamical system in the sense of Definition 3.1.

Earlier approaches to associating a Toeplitz-type crossed product to $(A, P, \alpha)$ involve a product system over $P$, see e.g. [11, Section 3]. Along the same lines, for each $p \in P$, let $E_{p}:=\alpha_{p}(A) A$ be the $C^{*}$-correspondence over $A$ where

$$
\langle x, y\rangle_{p}:=x^{*} y, \quad a \cdot x \cdot b:=\alpha_{p}(a) x b, \quad x, y \in E_{p}, a, b \in A .
$$


We define multiplication on $E_{\alpha}=\bigsqcup_{p \in P} E_{p}$ by

$$
E_{p} \times E_{q} \ni(x, y) \longmapsto \alpha_{q}(x) y \in E_{p q} .
$$

It is readily seen that the above map induces an isomorphism $E_{p} \otimes E_{q} \cong E_{p q}$ and hence $E_{\alpha}$ is a product system, cf. [11, Lemma 3.2]. The left action on each fiber is by generalized compacts, cf. [18, Lemma 3.25]. Hence by Lemma 2.4 we have a right-tensor $C^{*}$-precategory $\mathcal{K}_{E_{\alpha}}=\left\{\mathcal{K}\left(E_{p}, E_{q}\right)\right\}_{p, q \in P}$ which is a well-aligned ideal in the $C^{*}$-precategory associated to $E$.

We describe next another right-tensor $C^{*}$-precategory from $(A, P, \alpha)$ which will be useful in proving that our dynamical system is well-aligned. As in [17, Example 3.4], $\mathcal{K}_{\alpha}:=$ $\left\{\alpha_{p}(A) A \alpha_{q}(A)\right\}_{p, q \in P}$ is a $C^{*}$-precategory with multiplication, involution and norm inherited from $A$. There is a right tensoring on $\mathcal{K}_{\alpha}$ given by

$$
\alpha_{p}(A) A \alpha_{q}(A) \ni a \longrightarrow a \otimes 1_{r}:=\alpha_{r}(a) \in \alpha_{p r}(A) A \alpha_{q r}(A) .
$$

Our first observation is that $\mathcal{K}_{\alpha}$ and $\mathcal{K}_{E_{\alpha}}$ are the same, up to isomorphism.

Lemma 3.8. The right-tensor $C^{*}$-precategories $\mathcal{K}_{E_{\alpha}}$ and $\mathcal{K}_{\alpha}$ are isomorphic with the isomorphism given by $\mathcal{K}\left(E_{p}, E_{q}\right) \ni \Theta_{x, y} \longmapsto x y^{*} \in \mathcal{K}_{\alpha}(p, q)$.

Proof. Let $x_{i} \in E_{q}$ and $y_{i} \in E_{p}$, for $i=1, \ldots, n$. Since $\sum_{i=1}^{n} x_{i} y_{i}^{*} \in \alpha_{q}(A) A \alpha_{p}(A)$ we have

$$
\left\|\sum_{i=1}^{n} x_{i} y_{i}^{*}\right\|=\sup _{\substack{y \in \alpha_{p}(A) A \\\|y\|=1}}\left\|\sum_{i=1}^{n} x_{i} y_{i}^{*} y\right\|=\sup _{\substack{y \in E_{p} \\\|y\|=1}}\left\|\sum_{i=1}^{n} x_{i}\left\langle y_{i}, y\right\rangle\right\|=\left\|\sum_{i=1}^{n} \Theta_{x_{i}, y_{i}}\right\| .
$$

Thus $\mathcal{K}\left(E_{p}, E_{q}\right) \ni \Theta_{x, y} \mapsto x y^{*} \in \mathcal{K}_{\alpha}(p, q)$ extends to an isometric isomorphism, and straightforward calculations show that these maps form an isomorphism of $C^{*}$-precategories $\mathcal{K}_{E_{\alpha}}$ and $\mathcal{K}_{\alpha}$. Further, the maps intertwine right tensoring because for $x \in E_{q}, y, z \in E_{p}, w \in E_{r}$ we have $\alpha_{r}(x) \in E_{q r}$ and $\alpha_{r}(y) \in E_{p r}$, thus

$$
\left(\Theta_{x, y} \otimes 1_{r}\right)(z \cdot w)=x \cdot\langle y, z\rangle_{p} \cdot w=\alpha_{r}(x) \alpha_{r}\left(y^{*} z\right) w=\Theta_{\alpha_{r}(x), \alpha_{r}(y)} z \cdot w .
$$

Lemma 3.9. Let $(\pi, S)$ be a representation of $(A, P, \alpha)$ on a Hilbert space $H$.

(i) For every $p \in P, S_{p}$ is a partial isometry and

$$
\pi(a) S_{p}=S_{p} \pi(\alpha(a)), \quad \text { for all } a \in A .
$$

In particular, the projection $S_{p} S_{p}^{*}$ belongs to the commutant of $\pi(A)$ and

$$
\mathcal{K}_{(\pi, S)}(p, q)=S_{p} \pi\left(\alpha_{p}(A) A \alpha_{q}(A)\right) S_{q}^{*}, \quad \text { for all } p, q \in P
$$

(ii) For any approximate unit $\left\{\mu_{\lambda}\right\}$ in $A$ and all $p \in P$ we have $S_{p}^{*} S_{p}=s$ - $\lim _{\lambda \in \Lambda} \pi\left(\alpha_{p}\left(\mu_{\lambda}\right)\right)$. In particular, $\pi(a) S_{p}^{*} S_{p}=\pi(a)$ for all $a \in A \alpha_{p}(A)$;

(iii) The family of projections $\left\{S_{p}^{*} S_{p}\right\}_{p \in P^{o p}}$ forms a decreasing net, that is

$$
q=t p \Longrightarrow S_{q}^{*} S_{q} \leq S_{p}^{*} S_{p}
$$

(iv) Let $a \in \mathcal{K}_{(\pi, S)}(p, q), b \in \mathcal{K}_{(\pi, S)}(s, t)$ and $k \in \mathcal{K}_{(\pi, S)}\left(p q^{-1} r, t s^{-1} r\right)$, where $p, q, s, t, r \in P$ with $q P \cap s P=r P$. The pair $(a \cdot b, k)$ is a redundancy if and only if

$$
k=S_{p q^{-1} r} \pi\left(\alpha_{q^{-1} r}\left(a_{0}\right) \alpha_{s^{-1} r}\left(b_{0}\right)\right) S_{t s^{-1} r}^{*}
$$

where $a_{0} \in \alpha_{p}(A) A \alpha_{q}(A)$ and $b_{0} \in \alpha_{s}(A) A \alpha_{t}(A)$ are such that $a=S_{p} \pi\left(a_{0}\right) S_{q}^{*}, b=$ $S_{s} \pi\left(b_{0}\right) S_{t}^{*}$. 
Proof. Part (i) follows from [18, Proposition 3.12]. Part (ii) follows from (3.1), because $\pi$ is nondegenerate and therefore $\pi\left(\mu_{\lambda}\right)$ converges strongly to identity. To see part (iii) assume that $q=t p$, and notice that by part (ii) we have

$$
\begin{aligned}
\left(S_{q}^{*} S_{q}\right)\left(S_{p}^{*} S_{p}\right) & =\mathrm{s}-\lim _{\lambda \in \Lambda} \mathrm{s}-\lim _{\lambda^{\prime} \in \Lambda} \pi\left(\alpha_{q}\left(\mu_{\lambda}\right) \alpha_{p}\left(\mu_{\lambda}^{\prime}\right)\right)=\mathrm{s}-\lim _{\lambda \in \Lambda} \mathrm{s}-\lim _{\lambda^{\prime} \in \Lambda} \pi\left(\alpha_{p}\left(\alpha_{t}\left(\mu_{\lambda}\right) \mu_{\lambda}^{\prime}\right)\right) \\
& =\mathrm{s}-\lim _{\lambda \in \Lambda} \pi\left(\alpha_{p}\left(\alpha_{t}\left(\mu_{\lambda}\right)\right)\right)=\mathrm{s}-\lim _{\lambda \in \Lambda} \pi\left(\alpha_{q}\left(\mu_{\lambda}\right)\right)=S_{q}^{*} S_{q} .
\end{aligned}
$$

Let now $a, b$ and $k$ be as in part (iv). By part (i) there are $a_{0} \in \alpha_{p}(A) A \alpha_{q}(A)$ and $b_{0} \in$ $\alpha_{s}(A) A \alpha_{t}(A)$ such that $a=S_{p} \pi\left(a_{0}\right) S_{q}^{*}, b=S_{s} \pi\left(b_{0}\right) S_{t}^{*}$. By Remark 3.5, condition (3.3) determines $k$ uniquely. Thus it suffices to show that for $k:=S_{p q^{-1} r} \pi\left(\alpha_{q^{-1} r}\left(a_{0}\right) \alpha_{s^{-1} r}\left(b_{0}\right)\right) S_{t s^{-1} r}^{*}$, the pair $(a \cdot b, k)$ is a redundancy. This follows from the following computation:

$$
\begin{aligned}
a \cdot b \pi(c) S_{t s^{-1} r} & =\left(S_{p} \pi\left(a_{0}\right) S_{q}^{*}\right)\left(S_{s} \pi\left(b_{0}\right) S_{t}^{*}\right) \pi(c) S_{t} S_{s^{-1} r} \stackrel{(3.1)}{=} S_{p} \pi\left(a_{0}\right) S_{q}^{*} S_{s} \pi\left(b_{0} \alpha_{t}(c)\right) S_{s^{-1} r} \\
& \stackrel{(i)}{=} S_{p} \pi\left(a_{0}\right) S_{q}^{*} S_{r} \pi\left(\alpha_{s^{-1} r}\left(b_{0} \alpha_{t}(c)\right)\right)=S_{p} \pi\left(a_{0}\right) S_{q}^{*} S_{q} S_{q^{-1} r} \pi\left(\alpha_{s^{-1} r}\left(b_{0} \alpha_{t}(c)\right)\right) \\
& \stackrel{(i i)}{=} S_{p} \pi\left(a_{0}\right) S_{q^{-1} r} \pi\left(\alpha_{s^{-1} r}\left(b_{0} \alpha_{t}(c)\right)\right) \\
& \stackrel{(i)}{=} S_{p q^{-1} r} \pi\left(\alpha_{q^{-1} r}\left(a_{0}\right) \alpha_{s^{-1} r}\left(b_{0} \alpha_{t}(c)\right)\right) \\
& =S_{p q^{-1} r} \pi\left(\alpha_{q^{-1} r}\left(a_{0}\right) \alpha_{s^{-1} r}\left(b_{0}\right)\right) \pi\left(\alpha_{t s^{-1} r}(c) \stackrel{(3.1)}{=} k \pi(c) S_{t s^{-1} r} .\right.
\end{aligned}
$$

Proposition 3.10. There are bijective correspondences between:

(i) representations $(\pi, S)$ of the $C^{*}$-dynamical system $(A, P, \alpha)$;

(ii) nondegenerate right-tensor representations $\Psi$ of $\mathcal{K}_{\alpha}$ on a Hilbert space;

(iii) nondegenerate representations $\psi$ of the product system $E_{\alpha}$ on a Hilbert space.

Explicitly, these correspondences are determined by

$$
\Psi_{p, q}(a)=S_{p} \pi(a) S_{q}^{*}, \quad \text { for } a \in \mathcal{K}_{\alpha}(p, q), p, q \in P
$$

and

$$
\psi_{p}(x)=S_{p} \pi(x), \quad \text { for } x \in E_{p}=\alpha_{p}(A) A \text { and } S_{p}=s-\lim _{\lambda \in \Lambda} \psi_{p}\left(\alpha_{p}\left(\mu_{\lambda}\right)\right),
$$

where $\left\{\mu_{\lambda}\right\}$ is an approximate unit in $A$ and $p, q \in P$. In particular, there are canonical isomorphisms $\mathcal{T}\left(E_{\alpha}\right) \cong \mathcal{T}\left(\mathcal{K}_{\alpha}\right) \cong \mathcal{T}(A, P, \alpha)$.

Proof. By [18, Proposition 3.10], modulo [18, Lemma 3.25], for each $p \in P$ the formula for $\psi_{p}$ in (3.6) yields a bijective correspondence between representations $\left(\psi_{p}, \psi_{e}\right)$ of the $C^{*}$ correspondence $E_{p}$ and representations $\left(\pi, S_{p}\right)$ of the single endomorphism $\alpha_{p}$. Thus to establish the bijective correspondence between representations in (i) and (iii) it suffices to check the equivalence of semigroup laws. Suppose that $\psi$ is given by $(3.6)$ for a representation $(\pi, S)$ of $(A, P, \alpha)$. By Lemma 3.9,

$$
\psi_{p}(x) \psi_{q}(y)=S_{p} \pi(x) S_{q} \pi(y)=S_{p} S_{q} \pi\left(\alpha_{q}(x)\right) \pi(y)=S_{p q} \pi\left(\alpha_{q}(x) y\right)=\psi_{p q}(x \cdot y) .
$$

Hence, $\psi$ is a representation of $E_{\alpha}$. Conversely, if $\psi$ is a representation of $E_{\alpha}$ and $S$ is given by the strong limits in (3.6), then

$$
\begin{aligned}
S_{p} S_{q} & =\mathrm{s}-\lim _{\lambda \in \Lambda} \mathrm{s}-\lim _{\lambda^{\prime} \in \Lambda} \psi_{p}\left(\alpha_{p}\left(\mu_{\lambda}\right)\right) \psi_{q}\left(\alpha_{q}\left(\mu_{\lambda}^{\prime}\right)\right)=\mathrm{s}-\lim _{\lambda \in \Lambda} \mathrm{s}-\lim _{\lambda^{\prime} \in \Lambda} \psi_{p q}\left(\alpha_{q}\left(\alpha_{p}\left(\mu_{\lambda}\right) \mu_{\lambda}^{\prime}\right)\right) \\
& =\mathrm{s}-\lim _{\lambda \in \Lambda} \psi_{p q}\left(\alpha_{q}\left(\alpha_{p}\left(\mu_{\lambda}\right)\right)\right)=S_{p q} .
\end{aligned}
$$


This proves the bijective correspondence between representations in (i) and (iii). By virtue of Lemma 3.8, the correspondence between representations in (ii) and (iii) is given by Corollary 2.8. In particular, in view of (3.6), (2.1) translates to (3.5).

Proposition 3.11. The system $(A, P, \alpha)$ is well-aligned and the bijective correspondences in Proposition 3.10 respect Nica covariance of representations. In particular,

$$
\mathcal{N} \mathcal{T}\left(E_{\alpha}\right) \cong \mathcal{N} \mathcal{T}\left(\mathcal{K}_{\alpha}\right) \cong \mathcal{N} \mathcal{T}(A, P, \alpha)
$$

Moreover, a representation $(\pi, S)$ of $(A, P, \alpha)$ is Nica covariant if and only if $S$ is Nica covariant as a representation of $P$, i.e.:

$$
\left(S_{p} S_{p}^{*}\right)\left(S_{q} S_{q}^{*}\right)= \begin{cases}S_{r} S_{r}^{*}, & \text { if } q P \cap s P=r P \text { for some } r \in P, \\ 0, & \text { if } q P \cap s P=\emptyset .\end{cases}
$$

Proof. The first claim in the proposition follows from applying Proposition 3.10, Lemma 3.9 (iv), and Proposition 2.10, see (2.1). Chasing universal properties will give the claimed isomorphisms of Nica-Toeplitz algebras.

To prove the last claim of the proposition, let $(\pi, S), \psi$ and $\Psi$ be in the correspondence described in Proposition 3.10. Assume that $(\pi, S)$, and therefore also $\Psi$, is Nica covariant. Then for every $p, q \in P$ and $a \in \alpha_{p}(A) A \alpha_{p}(A), b \in \alpha_{q}(A) A \alpha_{q}(A)$ we have

$$
S_{p} \pi(a) S_{p}^{*} S_{q} \pi(b) S_{q}^{*}= \begin{cases}S_{r} \pi\left(\alpha_{p^{-1} r}(a) \alpha_{q^{-1} r}(b)\right) S_{r}^{*}, & \text { if } q P \cap s P=r P \text { for some } r \in P, \\ 0, & \text { if } q P \cap s P=\emptyset .\end{cases}
$$

Inserting in the above formula $a=\alpha_{p}\left(\mu_{\lambda}\right)$ and $b=\alpha_{q}\left(\mu_{\lambda}\right)$, where $\left\{\mu_{\lambda}\right\}$ is an approximate unit in $A$, and passing to strong limit gives

$$
\left(S_{p} S_{p}^{*}\right)\left(S_{q} S_{q}^{*}\right)= \begin{cases}S_{r}\left(S_{p^{-1} r}^{*} S_{p^{-1} r}\right)\left(S_{q^{-1} r}^{*} S_{q^{-1} r}\right) S_{r}^{*}, & \text { if } q P \cap s P=r P \text { for some } r \in P, \\ 0, & \text { if } q P \cap s P=\emptyset,\end{cases}
$$

by Lemma 3.9 (ii). By Lemma 3.9 we have $S_{r}\left(S_{p^{-1} r}^{*} S_{p^{-1} r}\right)=S_{r}$ and $\left(S_{q^{-1} r^{*}}^{*} S_{q^{-1} r}\right) S_{r}^{*}=S_{r}^{*}$. Hence we get (3.7).

Conversely, suppose that (3.7) holds. Let $a \in \alpha_{p}(A) A \alpha_{p}(A)$ and $b \in \alpha_{q}(A) A \alpha_{q}(A)$ for some $p, q \in P$. If $q P \cap s P=\emptyset$, then $S_{p} \pi(a) S_{p}^{*} S_{q} \pi(b) S_{q}^{*}=0$ because $S_{p}$ and $S_{q}$ have orthogonal ranges. Assume that $q P \cap s P=r P$. By appealing to Lemma 3.9 (ii) and (i), we get

$$
\begin{aligned}
S_{p} \pi(a) S_{p}^{*} S_{q} \pi(b) S_{q}^{*} & \stackrel{(3.7)}{=} S_{p} \pi(a) S_{p}^{*} S_{r} S_{r}^{*} S_{q} \pi(b) S_{q}^{*} \\
& =S_{p} \pi(a)\left(S_{p}^{*} S_{p}\right) S_{p^{-1} r} S_{q^{-1} r}^{*}\left(S_{q}^{*} S_{q}\right) \pi(b) S_{q}^{*} \\
& =S_{p} \pi(a) S_{p^{-1} r} S_{q^{-1} r}^{*} \pi(b) S_{q}^{*} \\
& =S_{r} \pi\left(\alpha_{p^{-1} r}(a) \alpha_{q^{-1} r}(b)\right) S_{r}^{*} .
\end{aligned}
$$

This, in conjunction with Lemma 3.8, proves Nica covariance of $\psi$. Hence $(\pi, S)$ is Nica covariant, by the first part of the proposition.

Let us notice that for $h \in P^{*}$ the endomorphism $\alpha_{h}$ is in fact an automorphism. Thus we have a group action $\left(P^{*}\right)^{o p} \ni h \mapsto \alpha_{h} \in \operatorname{Aut}(A)$ of the opposite group to $P^{*}$. Recall, cf. [22, Definition 2.15], that the group $\left\{\alpha_{h}\right\}_{h \in P^{* o p}}$ of automorphisms of $A$ is aperiodic if for every $h \in P^{*} \backslash\{e\}$ and every non-zero hereditary subalgebra $D$ of $A$ we have $\inf \left\{\left\|\alpha_{h}(a) a\right\|: a \in\right.$ $\left.D^{+},\|a\|=1\right\}=0$.

Lemma 3.12. The group $\left\{\alpha_{h}\right\}_{h \in P^{* o p}}$ is aperiodic if and only if the Fell bundle $\left\{E_{\alpha_{h}}\right\}_{h \in P^{*}}$ is aperiodic if and only if the group of automorphisms $\left\{\otimes 1_{h}\right\}_{h \in P^{*}}$ of $\mathcal{K}_{\alpha}$ is aperiodic. 
Proof. It is known, see [22, Theorem 2.9], that aperiodicity of $\left\{\alpha_{h}\right\}_{h \in P^{*}}$ is equivalent to the following condition: for every $h \in P^{*} \backslash\{e\}$, every $b \in A$ and every non-zero hereditary subalgebra $D$ of $A$ we have $\inf \left\{\left\|\alpha_{h}(a) b a\right\|: a \in D^{+},\|a\|=1\right\}=0$. The latter is exactly aperiodicity of $\left\{E_{\alpha_{h}}\right\}_{h \in P^{*}}$. Aperiodicity of $\left\{E_{\alpha_{h}}\right\}_{h \in P^{*}}$ is equivalent to aperiodicity of $\left\{\otimes 1_{h}\right\}_{h \in P^{*}}$ on $\mathcal{K}_{\alpha}$, by Proposition 2.18 and Lemma 3.8.

We are now ready to state the uniqueness theorem for Nica-Toeplitz crossed products associated to $(A, P, \alpha)$.

Theorem 3.13 (Uniqueness Theorem for $\mathcal{N} \mathcal{T}(A, P, \alpha))$. Let $(A, P, \alpha)$ be a $C^{*}$-dynamical system where each $\alpha_{p}, p \in P$, is an endomorphism, and $P$ is a right LCM semigroup. Suppose that either $P^{*}=\{e\}$ or that the group $\left\{\alpha_{h}\right\}_{h \in P^{* o p}}$ of automorphisms of $A$ is aperiodic. Assume moreover that $\mathcal{K}_{\alpha}$ is amenable. Then for a Nica covariant representation $(\pi, S)$ of $(A, P, \alpha)$, i.e. a representation satisfying (3.7), the canonical epimorphism:

$$
\mathcal{N} \mathcal{T}(A, P, \alpha) \longrightarrow \overline{\operatorname{span}}\left\{S_{p} \pi(a) S_{q}^{*}: a \in \alpha_{p}(A) A \alpha_{q}(A), p, q \in P\right\}
$$

is an isomorphism if and only if for any finite family $q_{1}, \ldots, q_{n} \in P \backslash P^{*}$ the representation $A \ni a \mapsto \pi(a) \prod_{i=1}^{n}\left(1-S_{q_{i}} S_{q_{i}}^{*}\right)$ is faithful.

Proof. By Proposition 3.11, we may view $\mathcal{N} \mathcal{T}(A, P, \alpha)$ as the Nica-Toeplitz algebra $\mathcal{N} \mathcal{T}\left(E_{\alpha}\right)$ of the compactly-aligned product system $E_{\alpha}$, where the left action on each fiber is by compacts. Thus the assertion follows from Theorem 2.19 modulo Lemma 3.12 and the observation that for a representation $\Psi$ of $\mathcal{K}_{\alpha}$ associated to $(\pi, S)$ we have, due to Lemma 3.9 (ii), $Q_{p}^{\Psi} H=$ $\Psi_{p, p}\left(\mathcal{K}_{\alpha}(p, p)\right) H=S_{p} \pi\left(\mathcal{K}_{\alpha}(p, p)\right) S_{p}^{*} H=S_{p} S_{p}^{*} H$.

3.3. Nica-Toeplitz crossed products by transfer operators. Throughout this section we assume that $L: P \ni p \mapsto L_{p} \in \operatorname{Pos}(A)$ is a unital semigroup antihomomorphism taking values in the semigroup of positive maps on a $C^{*}$-algebra $A$. We additionally assume that for each $p \in P$ the map $L_{p}: A \rightarrow A$ admits a 'multiplicative section', i.e. a $*$-homomorphism $\alpha_{p}: A \rightarrow M(A)$ such that

$$
L_{p}\left(a \alpha_{p}(b)\right)=L_{p}(a) b, \quad a, b \in A .
$$

Thus $\left(A, \alpha_{p}, L_{p}\right)$ is a so called Exel-system and $L_{p}$ is a (generalized) transfer operator for the endomorphism $\alpha_{p}$ [6], [9]. We emphasize that the choice of endomorphisms $\left\{\alpha_{p}\right\}_{p \in P}$ in general is far from being unique, cf. [18]. In particular, we do not assume that the family $\left\{\alpha_{p}\right\}_{p \in P}$ forms a semigroup. Nevertheless, we show that we may associate to $(A, P, L)$ a product system mimicking [25]. We also note that (3.8) implies that each $L_{p}$ is not only positive but in fact a completely positive map, cf. [18, Lemma 4.1]. Thus $(A, P, L)$ is a $C^{*}$-dynamical system in the sense of Definition 3.1.

Let $p \in P$. The $C^{*}$-correspondence $M_{p}$ associated to the transfer operator $L_{p}$ is the completion of the space $A_{p}:=A$ endowed with a right semi-inner-product $A$-bimodule structure given by

$$
a \cdot x \cdot b:=a x \alpha_{p}(b) \text { for } a, b \in A \text { and }\langle x, y\rangle_{p}:=L_{p}\left(x^{*} y\right) \text { for all } x, y, a \in A_{p} .
$$

The image of $x \in A_{p}=A$ in $M_{p}$ will be denoted by $(p, x)$.

Remark 3.14. By [18, Lemma 4.4], for each $p \in P$ the map $\left(p, a \alpha_{p}(b)\right) \mapsto a \otimes_{L_{p}} b, a, b \in A$, determines an isomorphism of $C^{*}$-correspondences from $M_{p}$ onto the KSGNS-correspondence $X_{L_{p}}$ of the completely positive map $L_{p}$ ([18, Lemma 4.4] is stated under the assumption that $\alpha_{p}(A) \subseteq A$, but a quick inspection of the proof shows that this assumption is not needed, cf. also Lemma 3.15 below). 
Lemma 3.15. Let $p, q \in P$. For any $a \in A, x \in A_{p q}$, and any approximate unit $\left\{\mu_{\lambda}\right\}$ in $A$ the elements $\left(p q, x \alpha_{p}\left(\mu_{\lambda}\right)\right)$ converge to $(p q, x)$ in $M_{p q}$.

Proof. This follows from taking limits in the equalities

$$
\begin{aligned}
\left\|\left(p q, x \alpha_{p}\left(\mu_{\lambda}\right)-x\right)\right\|^{2} & =\left\|L_{p q}\left(\alpha_{p}\left(\mu_{\lambda}\right) x^{*} x \alpha_{p}\left(\mu_{\lambda}\right)-\alpha_{p}\left(\mu_{\lambda}\right) x^{*} x-x^{*} x \alpha_{p}\left(\mu_{\lambda}\right)+x^{*} x\right)\right\| \\
& =\left\|L_{q}\left(\mu_{\lambda} L_{p}\left(x^{*} x\right) \mu_{\lambda}-\mu_{\lambda} L_{p}\left(x^{*} x\right)-L_{p}\left(x^{*} x\right) \mu_{\lambda}+L_{p}\left(x^{*} x\right)\right)\right\| .
\end{aligned}
$$

Proposition 3.16. The disjoint union of $C^{*}$-correspondences $M_{L}=\bigsqcup_{p \in P} M_{p}$ is a product system over the semigroup $P$, with multiplication determined by

$$
(p, x)(q, y):=\left(p q, x \alpha_{p}(y)\right), \quad x, y \in A, p, q \in P .
$$

Proof. For all $x, y, x^{\prime}, y^{\prime} \in A, p, q \in P$ we have

$$
\begin{aligned}
\left\langle(p, x) \otimes_{A}(q, y),\left(p, x^{\prime}\right) \otimes_{A}\left(q, y^{\prime}\right)\right\rangle & =\left\langle(q, y),\left\langle(p, x),\left(p, x^{\prime}\right)\right\rangle\left(q, y^{\prime}\right)\right\rangle \\
& =L_{q}\left(y^{*} L_{p}\left(x^{*} x^{\prime}\right) y^{\prime}\right)=L_{p q}\left(\alpha_{p}\left(y^{*}\right) x^{*} x \alpha_{p}(y)\right) \\
& =\left\langle\left(p q, x \alpha_{p}(y)\right),\left(p q, x^{\prime} \alpha_{p}\left(y^{\prime}\right)\right)\right\rangle \\
& =\left\langle(p, x)(q, y),\left(p, x^{\prime}\right)\left(q, y^{\prime}\right)\right\rangle .
\end{aligned}
$$

Thus we see that (3.9) extends uniquely to a multiplication $M_{p} \times M_{q} \rightarrow M_{p q}$ that factors trough to an isometric $C^{*}$-correspondence map $M_{p} \otimes_{A} M_{q} \rightarrow M_{p q}$, which is also surjective by Lemma 3.15. What is left to be shown is that multiplication (3.9) is associative. To this end, we note that for any $x, y, z \in A$ and $p, q, r \in P$ we have

$$
((p, x)(q, y))(r, z)=\left(p q r, x \alpha_{p}(y) \alpha_{q p}(z)\right), \quad(p, x)((q, y)(r, z))=\left(p q r, x \alpha_{p}\left(y \alpha_{q}(z)\right)\right) .
$$

Thus it suffices to show that $\left\|x \alpha_{p}(y) \alpha_{q p}(z)-x \alpha_{p}\left(y \alpha_{q}(z)\right)\right\|_{M_{p q r}}^{2}=0$. This however follows from the transfer property of $L$ since for any $a \in A$ we have

$$
\begin{aligned}
L_{p q r}\left(a x \alpha_{p}\left(y \alpha_{q}(z)\right)\right. & =L_{q r}\left(L_{p}(a x) y \alpha_{q}(z)\right)=L_{r}\left(L_{q}\left(L_{p}\left(a x \alpha_{p}(y)\right)\right) z\right) \\
& =L_{r}\left(L_{p q}\left(a x \alpha_{p}(y) \alpha_{q p}(z)\right)\right)=L_{p q r}\left(a x \alpha_{p}(y) \alpha_{q p}(z)\right) .
\end{aligned}
$$

Proposition 3.17. Let $M_{L}$ be the product system constructed above. We have a one-to-one correspondence between representations $(\pi, S)$ of $(A, P, L)$ and nondegenerate representations $\psi$ of $M_{L}$ on Hilbert spaces, given by

$$
\begin{gathered}
\psi_{p}(p, x)=\pi(x) S_{p}, \quad x \in A, \\
S_{p}=s-\lim _{\lambda \in \Lambda} \psi_{p}\left(p, \mu_{\lambda}\right)
\end{gathered}
$$

where $\left\{\mu_{\lambda}\right\}_{\lambda \in \Lambda}$ is an approximate unit in $A$. For the corresponding representations, we have $C^{*}(\pi, S)=C^{*}\left(\psi\left(M_{L}\right)\right)$, and in particular $\mathcal{T}(A, P, L) \cong \mathcal{T}\left(M_{L}\right)$.

Proof. In view of Remark 3.14, it follows from [18, Proposition 3.10] that relations (3.10) and (3.11) establish bijective correspondence between representations $\left(\pi, S_{p}\right)$ of $\left(A, L_{p}\right)$ and nondegenerate representations $\left(\psi_{e}, \psi_{p}\right)$ of $M_{p}$. Thus we only need to check the semigroup laws. Assume first that $(\pi, S)$ is a representation of $(A, L)$ and let $\psi$ be given by $(3.10)$. 
The isomorphism $M_{p} \cong X_{L_{p}}$ in Remark 3.14 implies that $\pi\left(a \alpha_{p}(b)\right) S_{p}=\psi_{p}\left(p, a \alpha_{p}(b)\right)=$ $\pi(a) S_{p} \pi(b)$ for any $a, b \in A$. Using this, for $x, y \in A, p, q \in P$, we get

$$
\psi(p, x) \psi(q, y)=\pi(x) S_{p} \pi(y) S_{q}=\pi\left(x \alpha_{p}(y)\right) S_{p q}=\psi\left(p q, x \alpha_{p}(y)\right) .
$$

Hence $\psi: M_{L} \rightarrow B(H)$ is a semigroup homomorphism.

Now assume that $\psi: M_{L} \rightarrow B(H)$ is a representation. By (3.10), (3.11) and Lemma 3.15 we have

$$
\begin{aligned}
S_{p} S_{q} & =\mathrm{s}-\lim _{\lambda \in \Lambda}\left(\mathrm{s}-\lim _{\lambda^{\prime} \in \Lambda^{\prime}} \psi\left(p, \mu_{\lambda}\right) \psi\left(q, \mu_{\lambda^{\prime}}^{\prime}\right)\right)=\mathrm{s}-\lim _{\lambda \in \Lambda}\left(\mathrm{s}-\lim _{\lambda^{\prime} \in \Lambda^{\prime}} \psi\left(p q, \mu_{\lambda} \alpha_{p}\left(\mu_{\lambda^{\prime}}^{\prime}\right)\right)\right) \\
& \left.=\mathrm{s}-\lim _{\lambda \in \Lambda} \psi\left(p q, \mu_{\lambda}\right)\right)=\mathrm{s}-\lim _{\lambda \in \Lambda} \pi\left(\mu_{\lambda}\right) S_{p q}=S_{p q} .
\end{aligned}
$$

Remark 3.18. If for $p \in P$ we may choose $\alpha_{p}$ taking values in $A$, then for each $p \in P, L_{p}$ extends to a strictly continuous map $\bar{L}_{p}: \mathcal{M}(A) \rightarrow \mathcal{M}(A)$, see [18, Proposition 4.2]. This implies that the limit (3.11) may be taken in the strict topology of $\mathcal{M}\left(C^{*}(\pi, S)\right)$ and so the multiplier $S_{p}$ is determined by $S_{p} \pi(a)=\psi_{p}\left(p, \alpha_{p}(a)\right)$, cf. [18, Proposition 3.10] and Lemma 3.3 .

Proposition 3.19. Suppose that the product system $M_{L}$ is compactly aligned. The bijective correspondence in Proposition 3.17 restricts to a bijective correspondence between NicaToeplitz representations $(\pi, S)$ of $(A, P, L)$ and nondegenerate Nica-Toeplitz representations $\psi$ of $M_{L}$. In particular,

$$
\mathcal{N} \mathcal{T}(A, P, L)=\overline{\operatorname{span}}\left\{j_{A}(a) \hat{s}_{p} \hat{s}_{q}^{*} j_{A}(b): a \in \alpha_{p}(A) A, b \in \alpha_{q}(A) A, p, q \in P\right\} \cong \mathcal{N} \mathcal{T}\left(M_{L}\right) .
$$

Proof. Let $(\pi, S)$ be a representation of $(A, P, L)$ and $\psi$ a representation of $M_{L}$ such that (3.10) and (3.11) hold. By Proposition 2.10, $\psi$ is Nica covariant if and only if the representation $\Psi:=\left\{\Psi_{p, q}\right\}_{p, q \in P}$ of $\mathcal{K}_{M_{L}}$, given by (2.1), is Nica covariant. Note that for every $p, q \in P$, the map $\Psi_{p, q}: \mathcal{K}\left(M_{q}, M_{p}\right) \rightarrow \overline{\pi(A) S_{p} \pi(A) S_{q}^{*} \pi(A)}$, given by (2.1), is surjective. Thus if $a \in \mathcal{K}_{(\pi, S)}(p, q), b \in \mathcal{K}_{(\pi, S)}(s, t)$ for some $p, q, s, t \in P$, then there are $a^{\prime} \in \mathcal{K}_{M_{L}}(p, q), b^{\prime} \in$ $\mathcal{K}_{M_{L}}(s, t)$ such that $\Psi_{p, q}\left(a^{\prime}\right)=a$ and $\Psi_{s, t}\left(b^{\prime}\right)=b$. It suffices to show that if $q P \cap s P=r P$, for some $r \in P$, then $(a \cdot b, k)$, where $k=\Psi_{p q^{-1} r, t^{-1} r}\left(\left(a^{\prime} \otimes 1_{q^{-1} r}\right)\left(b^{\prime} \otimes 1_{s^{-1} r}\right)\right)$, is a redundancy for $(\pi, S)$. For any $c \in A$ we have

$$
\begin{aligned}
b \pi(c) S_{t s^{-1} r} & =b \pi(c) S_{t} S_{s^{-1} r}=b \psi_{t}((t, c)) S_{s^{-1} r}=\psi_{s}\left(b^{\prime}(t, c)\right) S_{s^{-1} r} \\
& \stackrel{(3.11)}{=} \mathrm{s} \lim _{\lambda \in \Lambda} \psi_{s}\left(b^{\prime}(t, c)\right) \psi_{s^{-1} r}\left(\left(s^{-1} r, \mu_{\lambda}\right)\right) \\
& =\mathrm{s}-\lim _{\lambda \in \Lambda} \psi_{r}\left(b^{\prime}(t, c)\left(s^{-1} r, \mu_{\lambda}\right)\right) \\
& =\mathrm{s}-\lim _{\lambda \in \Lambda} \bar{\Psi}_{r, t s^{-1} r}\left(b^{\prime} \otimes 1_{s^{-1} r}\right) \psi_{t s^{-1} r}\left((t, c)\left(s^{-1} r, \mu_{\lambda}\right)\right) \\
& =\mathrm{s}-\lim _{\lambda \in \Lambda} \bar{\Psi}_{r, t s^{-1} r}\left(b^{\prime} \otimes 1_{s^{-1} r}\right) \pi(c) S_{t} \psi_{s^{-1} r}\left(\left(s^{-1} r, \mu_{\lambda}\right)\right) \\
& =\bar{\Psi}_{r, t s^{-1} r}\left(b^{\prime} \otimes 1_{s^{-1} r}\right) \pi(c) S_{t s^{-1} r} .
\end{aligned}
$$

Hence for any $c \in A$ we have $b \pi(c) S_{t s^{-1} r}=\bar{\Psi}_{r, t s^{-1} r}\left(b^{\prime} \otimes 1_{s^{-1} r}\right) \pi(c) S_{t s^{-1} r} \in \psi_{r}\left(M_{r}\right)$. Since, for any $x \in M_{r}$ we have $a \psi_{r}(x)=\bar{\Psi}_{p q^{-1} r, r}\left(a^{\prime} \otimes 1_{s^{-1} r}\right) \psi_{r}(x)$, we conclude that for any 
$c \in A$ we have $a \cdot b \pi(c) S_{t s^{-1} r}=\Psi_{p q^{-1} r, t s^{-1} r}\left(\left(a^{\prime} \otimes 1_{q^{-1} r}\right)\left(b^{\prime} \otimes 1_{s^{-1} r}\right)\right) \pi(c) S_{t s^{-1} r}$. Hence $\left(a \cdot b, \Psi_{p q^{-1} r, t s^{-1} r}\left(\left(a^{\prime} \otimes 1_{q^{-1} r}\right)\left(b^{\prime} \otimes 1_{s^{-1} r}\right)\right)\right)$ is a redundancy for $(\pi, S)$.

Using the above result we can apply Theorem 2.19 to the product system $M_{L}$ to get a uniqueness theorem for the Nica-Toeplitz crossed product $\mathcal{N} \mathcal{T}(A, P, L)$. Nevertheless, in this generality we can not simplify the assertion of Theorem 2.19 in a meaningful way. Therefore we will specialize to the case of 'transfer operators of finite type'.

3.4. Nica-Toeplitz crossed products by transfer operators of finite type. As in the previous subsection, we let $L: P \ni p \mapsto L_{p} \in \operatorname{Pos}(A)$ be a unital semigroup antihomomorphism. We recall that if $\varrho: A \rightarrow A$ is a positive map, then the multiplicative domain of $\varrho$ is the $C^{*}$-subalgebra of $A$ given by

$$
M D(\varrho):=\{a \in A: \varrho(b) \varrho(a)=\varrho(b a) \text { and } \varrho(a) \varrho(b)=\varrho(a b) \text { for every } b \in A\} .
$$

Throughout this subsection for every $p \in P$ we make the following standing assumptions:

(A1) $L_{p}$ faithful, i.e. $L_{p}\left(a^{*} a\right)=0$ implies $a=0$;

(A2) $L_{p}$ maps its multiplicative domain onto $A$.

We note that in the presence of axiom (A1), axiom (A2) is equivalent to the following two conditions:

(A2a) there is an endomorphism $\alpha_{p}: A \rightarrow A$ such that $L_{p}$ is a transfer operator for $\alpha_{p}$ as in [6] and $\mathbf{E}_{p}:=\alpha_{p} \circ L_{p}$ is a conditional expectation onto the range of $\alpha_{p}$;

(A2b) $L_{p}\left(\mu_{\lambda}\right)$ converges strictly to $1 \in \mathcal{M}(A)$, for any approximate unit $\left\{\mu_{\lambda}\right\}$ in $A$.

Specifically, (A1) and (A2) imply that $\left.L_{p}\right|_{M D\left(L_{p}\right)}$ is a $*$-isomorphism onto $A$ and its inverse:

$$
\alpha_{p}:=\left(\left.L_{p}\right|_{M D\left(L_{p}\right)}\right)^{-1}
$$

defines a monomorphism $\alpha_{p}$ with properties as in (A2a), cf. [18, Propositions 4.16]. Then property (A2b) follows from [18, Propositions 4.13], and we also have $\left\|L_{p}\right\|=1$, cf. [18, Lemma 2.1]. Conversely, properties (A2a), (A2b) imply (A2) by [18, Propositions 4.16], and then (A1) implies that $\alpha_{p}$ in (A2a) has to be of the form (3.12), see [18, Propositions 4.18].

Lemma 3.20. The maps in (3.12) form an action of $P$ by endomorphisms of $A$.

Proof. We need to prove that $\alpha_{p q}=\alpha_{p} \circ \alpha_{q}$ for all $p, q \in P$. We claim first that $L_{p}\left(M D\left(L_{p q}\right)\right) \subseteq$ $M D\left(L_{q}\right)$. Let $a \in A$ and $b \in M D\left(L_{q p}\right)$. Then

$$
L_{q}\left(L_{p}(b) a\right)=L_{q}\left(L_{p}\left(b \alpha_{p}(a)\right)\right)=L_{p q}\left(b \alpha_{p}(a)\right)=L_{p q}(b) L_{p q}\left(\alpha_{p}(a)\right)=L_{q}\left(L_{p}(b)\right) L_{q}(a),
$$

and similarly one gets $L_{q}\left(a L_{p}(b)\right)=L_{q}(a) L_{q}\left(L_{p}(b)\right)$.

Secondly, we show that $M D\left(L_{p q}\right) \subseteq M D\left(L_{p}\right)$. Indeed, since $L_{p}$ is a contractive completely positive map, we have

$$
M D\left(L_{p}\right)=\left\{a \in A: L_{p}\left(a^{*}\right) L_{p}(a)=L_{p}\left(a^{*} a\right) \text { and } L_{p}(a) L_{p}\left(a^{*}\right)=L_{p}\left(a a^{*}\right)\right\},
$$

cf. [18, Proposition 2.6]. Now, since $L_{p}\left(M D\left(L_{p q}\right)\right) \subseteq M D\left(L_{q}\right)$ and $L_{p q}=L_{q} \circ L_{p}$, for any $a \in M D\left(L_{p q}\right)$ we get

$$
L_{q}\left(L_{p}\left(a^{*}\right) L_{p}(a)\right)=L_{p q}\left(a^{*}\right) L_{p q}(a)=L_{p q}\left(a^{*} a\right)=L_{q}\left(L_{p}\left(a^{*} a\right)\right) .
$$

Faithfulness of $L_{q}$ implies that $L_{p}\left(a^{*}\right) L_{p}(a)=L_{p}\left(a^{*} a\right)$. Replacing $a$ with $a^{*}$, we get $L_{p}(a) L_{p}\left(a^{*}\right)=$ $L_{p}\left(a a^{*}\right)$. Hence $M D\left(L_{p q}\right) \subseteq M D\left(L_{p}\right)$.

Using the above inclusions, we conclude that $L_{p}$ restricts to a monomorphism $L_{p}: M D\left(L_{p q}\right) \rightarrow$ $M D\left(L_{q}\right)$. In fact, since $L_{p q}=L_{q} \circ L_{p}$ restricts to an isomorphism from $M D\left(L_{p q}\right)$ onto $A$ 
and $L_{q}$ restricts to an isomorphism from $M D\left(L_{q}\right)$ onto $A$, we see that $L_{p}: M D\left(L_{p q}\right) \rightarrow$ $M D\left(L_{q}\right)$ is an isomorphism. It is restriction of the isomorphism $L_{p}: M D\left(L_{p}\right) \rightarrow A$. Hence $\left.\left(\left.L_{p}\right|_{M D\left(L_{p}\right)}\right)^{-1}\right|_{M D\left(L_{q}\right)}=\left(\left.L_{p}\right|_{M D\left(L_{p q}\right)}\right)^{-1}$. Thus we obtain

$$
\begin{aligned}
\alpha_{p q} & =\left(\left.L_{p q}\right|_{M D\left(L_{p q}\right)}\right)^{-1}=\left(\left.\left.L_{q}\right|_{M D\left(L_{q}\right)} \circ L_{p}\right|_{M D\left(L_{p q}\right)}\right)^{-1}=\left(\left.L_{p}\right|_{M D\left(L_{p q}\right)}\right)^{-1} \circ\left(\left.L_{q}\right|_{M D\left(L_{q}\right)}\right)^{-1} \\
& =\left.\left(\left.L_{p}\right|_{M D\left(L_{p}\right)}\right)^{-1}\right|_{M D\left(L_{q}\right)} \circ\left(\left.L_{q}\right|_{M D\left(L_{q}\right)}\right)^{-1}=\alpha_{p} \circ \alpha_{q} .
\end{aligned}
$$

For each $p \in P, \mathbf{E}_{p}=\alpha_{p} \circ L_{p}$ is a faithful conditional expectation onto the multiplicative domain $M D\left(L_{p}\right)=\alpha_{p}(A)$ of $L_{p}$. We will assume that each $\mathbf{E}_{p}$ for $p \in P$ is of index-finite type as in [35]. Namely, for every $p \in P$ we assume that

(A3) there is a finite quasi-basis $\left\{u_{1}^{p}, \ldots, u_{m_{p}}^{p}\right\} \subseteq A$ for $\mathbf{E}_{p}$, for each $p \in P$, i.e. we have

$$
a=\sum_{i=1}^{m_{p}} u_{i}^{p} \mathbf{E}_{p}\left(\left(u_{i}^{p}\right)^{*} a\right), \quad \text { for all } a \in A .
$$

Associated to $(A, P, L)$ we have the product system $M_{L}$ of Proposition 3.16. Axiom (A1) implies that the map $A \ni a \mapsto(p, a) \in M_{p}$ is injective, for each $p \in P$. Axiom (A3) implies that the left action of $A$ on each $M_{p}, p \in P$, is by compacts because for $a, x \in A, p \in P$, a simple calculation using (3.13) gives

$$
\sum_{i=1}^{m_{p}} \Theta_{\left(p, u_{i}^{p}\right),\left(p, a^{*} u_{i}^{p}\right)}(p, x)=(p, a x) .
$$

Therefore the left action of $a$ on $M_{p}$ is given by the operator $\sum_{i=1}^{m_{p}} \Theta_{\left(p, u_{i}^{p}\right),\left(p, a^{*} u_{i}^{p}\right)} \in \mathcal{K}\left(M_{p}\right)$. By Lemma 2.4, the ideal $\mathcal{K}_{M_{L}}$ in $\mathcal{L}_{M_{L}}$ is invariant under right tensoring. Hence $\mathcal{K}_{M_{L}}$ is a right-tensor $C^{*}$-precategory itself.

Under the assumptions (A1)-(A3) it is possible to describe a new right-tensor $C^{*}$-precategory, isomorphic to $\mathcal{K}_{M_{L}}$, but admitting an explicit formula for the right tensoring. With this at hand, after invoking Propositions 3.19 and 2.10, we give a more explicit characterization of Nica covariance of a representation $(\pi, S)$ of $(A, P, L)$.

For each $p \in P$ denote by $\mathcal{K}_{p}$ the reduced $C^{*}$-basic construction associated to the conditional expectation $\mathbf{E}_{p}$ cf. [35, Subsection 2.1]. Thus $\mathcal{K}_{p}:=\mathcal{K}\left(\mathcal{E}_{p}\right)$ where $\mathcal{E}_{p}$ is the right Hilbert $\alpha_{p}(A)$ module obtained by completion of $A$ with respect to the norm induced by the sesquilinear form $\langle x, y\rangle_{\alpha_{p}(A)}=\mathbf{E}_{p}\left(x^{*} y\right), x, y \in A$. We recall that there is an injective left action of $A$ on $\mathcal{E}_{p}$, induced by multiplication in $A$. Thus we identify $A$ as a subalgebra of $\mathcal{L}\left(\mathcal{E}_{p}\right)$. The operator $\mathbf{E}_{p}: A \rightarrow A$ extends to an idempotent $e_{p} \in \mathcal{L}\left(\mathcal{E}_{p}\right)$, and then

$$
\mathcal{K}_{p}=\overline{\operatorname{span}}\left\{a e_{p} b: a, b \in A\right\} .
$$

For each $p, q \in P$ we equip the algebraic tensor product $A \odot A$ with the $\mathcal{K}_{q}$-valued sesquilinear form determined by

$$
\langle a \odot b, c \odot d\rangle_{p, q}:=b^{*} \alpha_{q}\left(L_{p}\left(a^{*} c\right)\right) e_{q} d, \quad a, b, c, d \in A .
$$

We let $\mathcal{K}_{L}(p, q)$ be the Hilbert $\mathcal{K}_{q}$-module arising as the completion of $A \odot A$ with the seminorm associated to the above sesquilinear form. We denote by $a \otimes_{p, q} b$ the image of a simple tensor $a \odot b$ in the space $\mathcal{K}_{L}(p, q)$.

Proposition 3.21. The family of Banach spaces $\mathcal{K}_{L}:=\left\{\mathcal{K}_{L}(p, q)\right\}_{p, q \in P}$ defined above form a right-tensor $C^{*}$-precategory where

$$
\left(a \otimes_{p, q} b\right)^{*}:=b \otimes_{q, p} a, \quad\left(a \otimes_{p, q} b\right) \cdot\left(c \otimes_{q, r} d\right):=a \alpha_{p}\left(L_{q}(b c)\right) \otimes_{p, r} d,
$$




$$
\left(a \otimes_{p, q} b\right) \otimes 1_{r}:=\sum_{i=1}^{m_{r}} a \alpha_{p}\left(u_{i}^{r}\right) \otimes_{p r, q r} \alpha_{q}\left(u_{i}^{r}\right)^{*} b,
$$

for all $a, b, c, d \in A, p, q, r \in P$. Moreover, if $M_{L}$ is the product system associated to $L$, then the map

$$
a \otimes_{p, q} b \longmapsto \Theta_{(p, a),\left(q, b^{*}\right)}, \quad a, b \in A,
$$

establishes an isomorphism of right-tensor $C^{*}$-precategories from $\mathcal{K}_{L}$ onto the right-tensor $C^{*}$-precategory $\mathcal{K}_{M_{L}}=\left\{\mathcal{K}\left(M_{q}, M_{p}\right)\right\}_{p, q \in P}$.

Proof. The strategy of the proof is to show that (3.16) yields an isometric isomorphism $\mathcal{K}_{L}(p, q) \cong \mathcal{K}\left(M_{q}, M_{p}\right)$ under which the right-tensor $C^{*}$-precategory operations from $\mathcal{K}_{M_{L}}$ translate to the prescribed formulas for $\mathcal{K}_{L}$. To this end, note that for any $p, q \in P$ the maps $\mathcal{H}:=\alpha_{q} \circ L_{p}$ and $\mathcal{V}:=\alpha_{p} \circ L_{q}$ form an interaction in the sense of [7, Definition 3.1]. Indeed, we have $\mathcal{H} \circ \mathcal{V}=\alpha_{q} \circ L_{p} \circ \alpha_{p} \circ L_{q}=\alpha_{q} \circ L_{q}=\mathbf{E}_{q}$, and thus $\mathcal{H} \circ \mathcal{V} \circ \mathcal{H}=\mathbf{E}_{q} \circ \mathcal{H}=\mathcal{H}$. For any $a, b \in A$ we get

$$
\mathcal{H}(\mathcal{V}(a) b)=\alpha_{q}\left(L_{p}\left(\alpha_{p}\left(L_{q}(a)\right) b\right)\right)=\alpha_{q}\left(L_{q}(a)\right) \cdot \alpha_{q}\left(L_{p}(b)\right)=\mathcal{H}(\mathcal{V}(a)) \mathcal{H}(b) .
$$

The other relations follow by symmetric arguments. Now, by [7, Proposition 5.4], for $x=$ $\sum_{i=1}^{n} a_{i} \otimes_{p, q} b_{i}, a_{i}, b_{i} \in A$, we have

$$
\|x\|_{\mathcal{K}(p, q)}=\left\|\left[\mathcal{H}\left(a_{i}^{*} a_{j}\right)\right]_{i, j}^{\frac{1}{2}}\left[\mathcal{H}\left(\mathcal{V}\left(b_{i} b_{j}^{*}\right)\right)\right]_{i, j}^{\frac{1}{2}}\right\|_{M_{n}(A)}=\|\left[\alpha_{q}\left(L_{p}\left(a_{i}^{*} a_{j}\right)\right]_{i, j}^{\frac{1}{2}}\left[\alpha_{q}\left(L_{q}\left(b_{i} b_{j}^{*}\right)\right)\right]_{i, j}^{\frac{1}{2}} \|_{M_{n}(A)} .\right.
$$

Using the fact that $\alpha_{q}$ amplifies to an isometric $*$-homomorphism on $M_{n}(A)$ we get

$$
\begin{aligned}
\|x\|_{\mathcal{K}(p, q)} & =\left\|\left[L_{p}\left(a_{i}^{*} a_{j}\right)\right]_{i, j}^{\frac{1}{2}}\left[L_{q}\left(b_{i} b_{j}^{*}\right)\right]_{i, j}^{\frac{1}{2}}\right\|_{M_{n}(A)} \\
& =\left\|\left[\left\langle\left(p, a_{i}\right),\left(p, a_{j}\right)\right\rangle_{p}\right]_{i, j}^{\frac{1}{2}}\left[\left\langle\left(q, b_{i}^{*}\right),\left(q, b_{j}^{*}\right)\right\rangle_{q}\right]_{i, j}^{\frac{1}{2}}\right\|_{M_{n}(A)} .
\end{aligned}
$$

Comparing this with the norm of the operator $\sum_{i=1}^{n} \Theta_{\left(p, a_{i}\right),\left(q, b_{i}^{*}\right)}$ described in [16, Lemma 2.1], we finally arrive at $\left\|\sum_{i=1}^{n} a_{i} \otimes_{p, q} b_{i}\right\|_{\mathcal{K}_{L}(p, q)}=\left\|\sum_{i=1}^{n} \Theta_{\left(p, a_{i}\right),\left(q, b_{i}^{*}\right)}\right\|_{\mathcal{K}\left(M_{q}, M_{p}\right)}$. Thus (3.16) defines a linear isometry.

The standard formulas: $\Theta_{x, y}^{*}=\Theta_{y, x}, \Theta_{x, y} \circ \Theta_{z, v}=\Theta_{x\langle y, z\rangle_{q}, v}$ for $x \in M_{p}, y, z \in M_{q}$, and $v \in M_{r}$, translate via (3.16) to (3.14). Hence relations (3.14) indeed define a $C^{*}$-precategory structure on $\mathcal{K}_{L}$, and $\mathcal{K}_{L}$ is isomorphic to $\mathcal{K}_{M_{L}}$ as a $C^{*}$-precategory. Thus it remains to show that the right tensoring in $\mathcal{K}_{M_{L}}$ translates to (3.15) on the level of $\mathcal{K}_{L}$.

Note that the product system $M_{L}$ is (left) essential. Let $a, b, x, y \in A, p, q, r \in P$, and $T=\Theta_{(p, a),\left(q, b^{*}\right)}$. Taking into account that $(p, x) \otimes_{A}(q, y)=\left(p q, x \alpha_{p}(y)\right)$ we get

$$
\begin{aligned}
\left(T \otimes 1_{r}\right)(q, x) \otimes_{A}(r, y) & =\left(p, a \alpha_{p}\left(L_{q}(b x)\right)\right) \otimes_{A}(r, y)=\left(p r, a \alpha_{p}\left(L_{q}(b x) y\right)\right) \\
& =\left(p r, a \alpha_{p}\left(\sum_{i=1}^{m_{r}} u_{i}^{r}\left(\alpha_{r} \circ L_{r}\right)\left(\left(u_{i}^{r}\right)^{*} L_{q}\left(b x \alpha_{q}(y)\right)\right)\right)\right. \\
& =\left(p r, \sum_{i=1}^{m_{r}} a \alpha_{p}\left(u_{i}^{r}\right) \alpha_{p r}\left(L_{q r}\left(\alpha_{q}\left(u_{i}^{r}\right)^{*} b x \alpha_{q}(y)\right)\right)\right) \\
& =\left(p r,\left(\sum_{i=1}^{m_{r}} \Theta_{\left(p r, a \alpha_{p}\left(u_{i}^{r}\right)\right),\left(q r, b^{*} \alpha_{q}\left(u_{i}^{r}\right)\right)}\right) x \alpha_{q}(y)\right) \\
& =\left(\sum_{i=1}^{m_{r}} \Theta_{\left(p r, a \alpha_{p}\left(u_{i}^{r}\right)\right),\left(q r, b^{*} \alpha_{q}\left(u_{i}^{r}\right)\right)}\right)(q, x) \otimes_{A}(r, y) .
\end{aligned}
$$


Thus $\Theta_{(p, a),\left(q, b^{*}\right)}=\left(\sum_{i=1}^{m_{r}} \Theta_{\left(p r, a \alpha_{p}\left(u_{i}^{r}\right)\right),\left(q r, b^{*} \alpha_{q}\left(u_{i}^{r}\right)\right)}\right)$ and therefore (3.15) defines the desired right tensoring on $\mathcal{K}_{L}$.

Remark 3.22. In view of Proposition 3.21, since the image of $A \alpha_{p}(A)$ in $M_{p}$ is a dense subspace of $M_{p}$, cf. Lemma 3.15 , we have that $\mathcal{K}_{L}(p, q)=\overline{\operatorname{span}}\left\{a \otimes_{p, q} b: a \in A \alpha_{p}(A), b \in\right.$ $\left.\alpha_{q}(A) A\right\}$.

Lemma 3.23. Let $p P \cap q P=r P$. For any $a, b, c, d \in A$ we have

$$
\left(\left(a \otimes_{p, p} b\right) \otimes 1_{p^{-1} r}\right) \cdot\left(\left(c \otimes_{q, q} d\right) \otimes 1_{q^{-1} r}\right)=\sum_{i=1}^{m_{q^{-1} r}} a \mathbf{E}_{p}\left(b c \alpha_{q}\left(u_{i}^{q^{-1} r}\right)\right) \otimes_{r, r} \alpha_{q}\left(u_{i}^{q^{-1} r}\right)^{*} d .
$$

Proof. By (3.14) and (3.15), the left hand side of (3.17) is equal to

$$
\begin{aligned}
& \sum_{i=1}^{m_{p^{-1}} r}\left(a \alpha_{p}\left(u_{i}^{p^{-1} r}\right) \otimes_{r, r} \alpha_{p}\left(u_{i}^{p^{-1} r}\right)^{*} b\right) \cdot \sum_{i=1}^{m_{q^{-1} r}}\left(c \alpha_{q}\left(u_{i}^{q^{-1} r}\right) \otimes_{r, r} \alpha_{q}\left(u_{i}^{q^{-1} r}\right)^{*} d\right) \\
& \quad=\sum_{i=1, j=1}^{m_{p^{-1}}, m_{q^{-1} r}} a \alpha_{p}\left(u_{i}^{p^{-1} r}\right) \mathbf{E}_{r}\left(\alpha_{p}\left(u_{i}^{p^{-1} r}\right)^{*} b c \alpha_{q}\left(u_{j}^{q^{-1} r}\right)\right) \otimes_{r, r} \alpha_{q}\left(u_{i}^{q^{-1} r}\right)^{*} d .
\end{aligned}
$$

However, using that $\mathbf{E}_{r}=\alpha_{p} \circ \mathbf{E}_{p^{-1} r} \circ L_{p}$, for any $f \in A$, it follows that

$$
\begin{aligned}
\sum_{i=1}^{m_{p^{-1} 1_{r}}} \alpha_{p}\left(u_{i}^{p^{-1} r}\right) \mathbf{E}_{r}\left(\alpha_{p}\left(u_{i}^{p^{-1} r}\right)^{*} f\right) & =\sum_{i=1}^{m_{p^{-1} r}} \alpha_{p}\left(u_{i}^{p^{-1} r} \mathbf{E}_{p^{-1} r}\left(u_{i}^{p^{-1} r *} L_{p}(f)\right)\right) \\
& =\alpha_{p}\left(L_{p}(f)\right)=\mathbf{E}_{p}(f) .
\end{aligned}
$$

Now inserting $f=b c \alpha_{q}\left(u_{j}^{q^{-1} r}\right)$ in the computations above gives the assertion.

Proposition 3.24. A representation $(\pi, S)$ of $(A, P, L)$ is Nica covariant if and only if for every $p, q \in P$, and $a \in \alpha_{p}(A) A \alpha_{q}(A)$ the following are satisfied:

$$
S_{p}^{*} \pi(a) S_{q}=0 \quad \text { if } \quad p P \cap q P=\emptyset
$$

and

$$
S_{p} S_{p}^{*} \pi(a) S_{q} S_{q}^{*}=\sum_{i=1}^{m_{q^{-1} r}} \pi\left(\mathbf{E}_{p}\left(a \alpha_{q}\left(u_{i}^{q^{-1} r}\right)\right)\right) S_{r} S_{r}^{*} \pi\left(u_{i}^{q^{-1} r}\right)^{*} \quad \text { if } \quad p P \cap q P=r P .
$$

Proof. By Propositions 2.8, 3.19 and 3.21, there is a one-to-one correspondence between representations $(\pi, S)$ of $(A, P, L)$ and right-tensor representations $\Psi$ of $\mathcal{K}_{L}$ determined by

$$
\Psi\left(a \otimes_{p, q} b\right)=\pi(a) S_{p} S_{q}^{*} \pi(b), \quad a, b \in A .
$$

Moreover, $(\pi, S)$ is Nica covariant if and only if $\Psi$ is, cf. Proposition 2.10 .

Let $(\pi, S)$ be a Nica covariant representation. Thus the associated $\Psi$ in (3.20) is Nica covariant, too. Let $p, q \in P$, and $a \in \alpha_{p}(A) A \alpha_{q}(A)$. If $p P \cap q P=\emptyset$, then for any $b, c, d \in A$ we have

$$
0=\Psi\left(b \otimes_{p, p} a\right) \Psi\left(c \otimes_{q, q} d\right)=\pi(b) S_{p} S_{p}^{*} \pi(a c) S_{q} S_{q}^{*} \pi(d) .
$$

Letting $b, c, d \in A$ run through an approximate unit in $A$ and taking (strong) limit, we get $S_{p} S_{p}^{*} \pi(a) S_{q} S_{q}^{*}=0$, which is equivalent to $S_{p}^{*} \pi(a) S_{q}=0$. Let now $p P \cap q P=r P$. Invoking Lemma 3.23, with the roles of $a$ and $b$ exchanged, we get

$\pi(b) S_{p} S_{p}^{*} \pi(a c) S_{q} S_{q}^{*} \pi(d)=\Psi\left(\left(b \otimes_{p, p} a\right) \cdot\left(c \otimes_{q, q} d\right)\right)=\Psi\left(\left(\left(b \otimes_{p, p} a\right) \otimes 1_{p^{-1} r}\right) \cdot\left(\left(c \otimes_{q, q} d\right) \otimes 1_{q^{-1} r}\right)\right)$ 


$$
=\pi(b) \sum_{i=1}^{m_{q^{-1} r}} \pi\left(\mathbf{E}_{p}\left(a c \alpha_{q}\left(u_{i}^{q^{-1} r}\right)\right)\right) S_{r} S_{r}^{*} \pi\left(u_{i}^{q^{-1} r}\right)^{*} \pi(d) .
$$

Letting $b, c, d \in A$ run through an approximate unit in $A$ and taking (strong) limit gives (3.19).

Conversely, relations (3.18) and (3.19) imply Nica covariance as a reversal of the above arguments shows.

For $h \in P^{*}$, the mapping $L_{h}$ is invertible and hence by (A1) and (A2) we have $M D\left(L_{h}\right)=$ $A$, which means that $L_{h}$ is an automorphism of $A$ : we have $L_{h}=\alpha_{h^{-1}}=\alpha_{h}^{-1}$.

Theorem 3.25 (Uniqueness Theorem for $\mathcal{N} \mathcal{T}(A, P, L))$. Let $(A, P, L)$ be a $C^{*}$-dynamical system satisfying (A1), (A2), (A3) above. Suppose that either $P^{*}=\{e\}$ or that the group $\left\{\alpha_{h}\right\}_{h \in P^{*}}$ of automorphisms of $A$ is aperiodic. Assume also that $\mathcal{K}_{L}$ is amenable. Let $(\pi, S)$ be a Nica covariant representation of $(A, P, L)$. For each $p \in P$, let $Q_{p}$ be the projection onto the space $\overline{\pi(A) S_{p} H}$. Then the canonical surjective $*$-homomorphism

$$
\mathcal{N} \mathcal{T}(A, P, \alpha) \longrightarrow \overline{\operatorname{span}}\left\{\pi(a) S_{p} S_{q}^{*} \pi(b): a, b \in A, p, q \in P\right\}
$$

is an isomorphism if and only if for all finite families $q_{1}, \ldots, q_{n} \in P \backslash P^{*}$ the representation $A \ni a \mapsto \pi(a) \prod_{i=1}^{n}\left(1-Q_{q_{i}}\right)$ is faithful.

Proof. Note that $\left\{\pi(a) S_{p} S_{q}^{*} \pi(b): a, b \in A, p, q \in P\right\}$ is closed under multiplication due to Proposition 3.24. By Proposition 3.19, $\mathcal{N} \mathcal{T}(A, P, L)$ may be viewed as $\mathcal{N} \mathcal{T}\left(M_{L}\right)$. For $h \in P^{*}$, the automorphism $L_{h}$ of $A$ induces an isomorphism of $C^{*}$-correspondences $E_{\alpha_{h}}=$ $E_{L_{h^{-1}}} \cong M_{L_{h}}$. Thus by Lemma 3.12, the group $\left\{\alpha_{h}\right\}_{h \in P^{*}}=\left\{L_{h}\right\}_{h \in P^{* o p} \text { of automorphisms }}$ of $A$ is aperiodic if and only if the Fell bundle $\left\{M_{L_{h}}\right\}_{h \in P^{*}}$ is aperiodic. Recalling that under assumption (A3), the left action is by generalized compacts in each fiber, the assertion follows from Theorem 2.19 modulo Proposition 3.21 and the fact that for the representation $\Psi$ of $\mathcal{K}_{L}$ associated to $(\pi, S)$ and every $p \in P$ we have $\Psi_{p, p}\left(\mathcal{K}_{L}(p, p)\right) H=\pi(A) S_{p} S_{p}^{*} \pi(A) H=$ $\overline{\pi(A) S_{p} H}$.

\section{4. $C^{*}$-ALGEBRAS ASSOCIATED TO RIGHT LCM SEMigRoups}

Throughout this section we use the notation $S$ for a generic right LCM semigroup and reserve $P$ for semigroups in semidirect products as in [2]. Associated to any right LCM semigroup $S$ there is a universal $C^{*}$-algebra $C^{*}(S)=\overline{\operatorname{span}}\left\{v_{s} v_{t}^{*}: s, t \in S\right\}$ generated by an isometric representation $v$ of $S$ such that

$$
\left(v_{p} v_{p}^{*}\right)\left(v_{q} v_{q}^{*}\right)= \begin{cases}v_{r} v_{r}^{*} & \text { if } p P \cap q P=r P \\ 0 & \text { if } p P \cap q P=\emptyset .\end{cases}
$$

See [27] for the abstract construction of $C^{*}(S)$ valid for arbitrary left cancellative semigroups, and [1] or [30] for the case of right LCM semigroups. When $S$ is a right LCM semigroup, [2, Corollary 7.11] implies that $C^{*}(S)$ is isomorphic to the Nica-Toeplitz algebra $\mathcal{N} \mathcal{T}(X)$ for the compactly aligned product system $X$ over $S$ with fibers $X_{s} \cong \mathbb{C}$ for all $s \in S$. It is therefore natural to ask if Theorem 2.19 can be applied. On one hand, since the left action is by generalized compact operators in every fiber $X_{s}$, for $s \in S$, we will have equivalence of the three assertions (i)-(iii) if $\mathcal{K}_{X}$ is amenable and $S^{*}=\{e\}$. This in particular recovers the case (1) in [1, Theorem 4.3]. On the other hand, in case that $S^{*} \neq\{e\}$, the Fell bundle $\left\{X_{h}\right\}_{h \in S^{*}}$ can never be aperiodic because $X_{h}=\mathbb{C}$ for all $h \in S^{*}$. Therefore viewing $C^{*}(S)$ as a Nica-Toeplitz $C^{*}$-algebra associated to the product system $X$ with trivial (thus small) 
fibers we can not apply Theorem 2.19 when $S^{*} \neq\{e\}$. A possible solution to this obstacle is to consider $C^{*}(S)$ as a Nica-Toeplitz $C^{*}$-algebra associated to another product system with larger fibers, so that we can detect aperiodicity. For instance, given a controlled function into a group one could obtain a uniqueness result in terms of Fell bundles based on Proposition 2.20. However, in general the fibers of the arising Fell bundle will be very large. We propose an intermediate approach in the case that $S$ is a semidirect product of an LCM semigroup and a group. We will show useful alternative realizations of $C^{*}(S)$ as Nica-Toeplitz algebras associated to product systems with larger fibers over a smaller semigroup, which lead to efficient uniqueness results.

4.1. Semidirect products of LCM semigroups. Even though left and right semidirect products are equivalent as abstract constructions, it turns out that right semidirect products have rather different properties than the left semidirect products of a group by a semigroup considered for instance in $[1,2]$. To exemplify, we will use right semidirect products for actions of semigroups on groups to construct right LCM semigroups $S$ with non-trivial group of units $S^{*}$ and which are not necessarily right cancellative (see Proposition 4.3 below). Moreover, for these examples the constructible right ideals depend only the acting semigroup, unlike the case of left semidirect products.

We begin by fixing our conventions for the two constructions. For a semigroup $T$ we let End $T$ denote the semigroup of all semigroup homomorphisms $T \rightarrow T$ that preserve the identity $e_{T}$ in $T$. The identity endomorphism in $\operatorname{End} T$ is $\operatorname{id}_{T}$. A left action $P \stackrel{\theta}{\curvearrowright} T$ of a semigroup $P$ on $T$ is a unital semigroup homomorphism $\theta: P \rightarrow$ End $T$. A right action $T \stackrel{\vartheta}{\curvearrowleft} P$ is a unital semigroup antihomomorphism $\vartheta: P \rightarrow \operatorname{End} T$, i.e. $\vartheta_{p} \vartheta_{q}=\vartheta_{q p}$ for all $p, q \in P$.

Definition 4.1. Let $T, P$ be semigroups. The (left) semidirect product of $T$ by $P$ with respect to a left action $P \stackrel{\theta}{\curvearrowright} T$, denoted $T \rtimes_{\theta} P$, is the semigroup $T \times P$ with composition given by

$$
(g, p)(h, q)=\left(g \theta_{p}(h), p q\right), \quad \text { for } g, h \in T \text { and } p, q \in P \text {. }
$$

The (right) semidirect product of $T$ by $P$ with respect to a right action $T \stackrel{\vartheta}{\curvearrowleft} P$, denoted $P_{\vartheta} \ltimes T$, is the semigroup $P \times T$ with composition given by

$$
(p, g)(q, h)=\left(p q, \vartheta_{q}(g) h\right), \quad \text { for } g, h \in T \text { and } p, q \in P .
$$

Remark 4.2. The opposite semigroup $P^{o p}$ to a semigroup $P$ coincides with $P$ as a set but has multiplication defined by reversing the factors. Treating the corresponding endomorphisms as maps on the same set, we have End $P=$ End $P^{o p}$. Thus every right action $T \stackrel{\vartheta}{\curvearrowleft} P$ can be treated as the left action $P^{o p} \stackrel{\vartheta}{\curvearrowright} T^{o p}$, and there is an isomorphism of semigroups

$$
P_{\vartheta} \ltimes T \ni(p, g) \rightarrow(g, p) \in\left(T^{o p} \rtimes_{\vartheta} P^{o p}\right)^{o p} .
$$

The following proposition should be compared with [1, Lemma 2.4] proved for left semidirect products. It shows that right semidirect products in the realm of right LCM semigroups are always left cancellative and have easier structure of principal right ideals.

Proposition 4.3. Suppose that $G \stackrel{\vartheta}{\curvearrowleft} P$ is a right action of a right LCM semigroup $P$ with identity on a group $G$. Then $P_{\vartheta} \ltimes G$ is a right $L C M$ semigroup such that

$$
J(P) \cong J\left(P_{\vartheta} \ltimes G\right) \quad \text { and } \quad\left(P_{\vartheta} \ltimes G\right)^{*}=P^{*}{ }_{\vartheta} \ltimes G .
$$

Moreover, $P_{\vartheta} \ltimes G$ is cancellative if and only if $P$ is cancellative and every $\vartheta_{p}$ is injective, $p \in P$. 
Proof. The element $\left(e_{P}, e_{G}\right)$ is the identity of $P_{\vartheta} \ltimes G$. If $(p, g)(q, h)=(p, g)\left(q^{\prime}, h^{\prime}\right)$ then $p q=$ $p q^{\prime}$ and $\vartheta_{q}(g) h=\vartheta_{q^{\prime}}(g) h^{\prime}$. By left cancellation in $P$ we get $q=q^{\prime}$ and therefore also $h=h^{\prime}$. Thus $P_{\vartheta} \ltimes G$ is left cancellative. Since the action of the group $G$ on itself is transitive, we have $(p, g)\left(P_{\vartheta} \ltimes G\right)=(p P) \times G$ for every $(p, g) \in P_{\vartheta} \ltimes G$. Hence $P \ltimes{ }_{\vartheta} G$ is a right LCM semigroup with the semilattice of principal right ideals isomorphic to that of $P$, with isomorphism given by $p P \mapsto(p P) \ltimes G$. Plainly, relations $(p, g)(q, h)=(q, h)(p, q)=\left(e_{P}, e_{G}\right)$ hold if and only if $p \in P^{*}, q=p^{-1}$ and $h=\vartheta_{p^{-1}}\left(g^{-1}\right)$. This immediately gives $\left(P_{\vartheta} \ltimes G\right)^{*}=P^{*}{ }_{\vartheta} \ltimes G$.

The claim about $P_{\vartheta} \ltimes G$ being right cancellative follows by noting that $(p, g)(q, h)=$ $\left(p^{\prime}, g^{\prime}\right)(q, h)$ if and only if $p q=p^{\prime} q$ and $\vartheta_{q}(g)=\vartheta_{q}\left(g^{\prime}\right)$.

Remark 4.4. Using the last part of Proposition 4.3 it is easy to construct examples of not cancellative LCM semigroups from cancellative ones.

In general the (left) semidirect product of a group $G$ by a right LCM semigroup $P$ with respect to a left action $P \stackrel{\theta}{\curvearrowright} G$ is not an LCM semigroup. As introduced in [2, Definition 2.1], an algebraic dynamical system is a triple $(G, P, \theta)$ where $G$ is a group, $P$ is a right LCM semigroup, and $P \stackrel{\theta}{\curvearrowright} G$ is a left action by injective endomorphisms of $G$ which respects the order, i.e. $\theta_{p}(G) \cap \theta_{q}(G)=\theta_{r}(G)$ whenever for $p, q \in P$ there is $r \in P$ such that $p P \cap q P=r P$. By [1, Proposition 8.2 and Lemma 2.4], whenever $(G, P, \theta)$ is an algebraic dynamical system, the left semidirect product $P \rtimes_{\theta} G$ is a right LCM semigroup and $\left(P \rtimes_{\theta} G\right)^{*}=P^{*} \rtimes_{\theta} G$.

4.2. Semigroup $C^{*}$-algebras associated to right semidirect products $P_{\vartheta} \ltimes G$. Here we assume that $\vartheta$ is a right action of a right LCM semigroup $P$ on a group $G$. We let $\delta_{g}$ for $g \in G$ be the generating unitaries in $C^{*}(G)$.

Proposition 4.5. Let $\vartheta$ be a right action of a right LCM semigroup $P$ on a group $G$. There is an antihomomorphism $\alpha$ of $P$ into End $C^{*}(G)$ given by $\alpha_{p}\left(\delta_{g}\right)=\delta_{\vartheta_{p}(g)}$ for $g \in G$ and $p \in P$. Further, $\left(C^{*}(G), P, \alpha\right)$ is a $C^{*}$-dynamical system as in subsection 3.2, and $C^{*}\left(P_{\vartheta} \ltimes G\right) \cong$ $\mathcal{N} \mathcal{T}\left(C^{*}(G), P, \alpha\right)$.

Proof. We only prove the last assertion as the rest is routine. Proposition 3.11 provides natural isomorphisms between three different $C^{*}$-algebras associated to $\left(C^{*}(G), P, \alpha\right)$. We aim to show that $C^{*}\left(P_{\vartheta} \ltimes G\right)$ is isomorphic to the Nica-Toeplitz algebra $\mathcal{N} \mathcal{T}\left(E_{\alpha}\right)$ associated to the product system $E_{\alpha}$ with multiplication defined in (3.4).

Let $i_{E_{\alpha}}$ be the universal Nica covariant representation of $E_{\alpha}$. For each $p \in P$, denote $i_{p}$ the restriction of $i_{E_{\alpha}}$ to $E_{p}$. We claim that $w_{(p, g)}:=i_{p}\left(\delta_{g}\right)$ for $(p, g) \in P_{\vartheta} \ltimes G$ is a Li-family in $\mathcal{N} \mathcal{T}\left(E_{\alpha}\right)$. Let $(p, g),(q, h) \in P_{\vartheta} \ltimes G$. Then

$$
w_{(p, g)} w_{(q, h)}=i_{p}\left(\delta_{g}\right) i_{q}\left(\delta_{h}\right)=i_{p q}\left(\alpha_{q}\left(\delta_{g}\right) \delta_{h}\right)=w_{\left(p q, \vartheta_{q}(g) h\right)}
$$

and since $w_{(e, e)}=1$, we have a representation of $P_{\vartheta} \ltimes G$. Each $w_{(p, g)}$ is an isometry because $w_{(p, g)}^{*} w_{(p, g)}=i_{p}\left(\delta_{g}\right)^{*} i_{p}\left(\delta_{g}\right)=i_{e}\left(\left\langle\delta_{g}, \delta_{g}\right\rangle_{p}\right)=1$. Next we compute $w_{(p, g)}^{*} w_{(q, h)}$. Since $(p, g)\left(P_{\vartheta} \ltimes G\right) \cap(q, h)\left(P_{\vartheta} \ltimes G\right)=\emptyset$ if and only if $p P \cap q P=\emptyset$, in which case $i_{p}\left(\delta_{g}\right)^{*} i_{q}\left(\delta_{h}\right)=0$ by Nica covariance of $i_{E_{\alpha}}$. Thus $w_{(p, g)}^{*} w_{(q, h)}=0$ when $(p, g)\left(P_{\vartheta} \ltimes G\right) \cap(q, h)\left(P_{\vartheta} \ltimes G\right)=\emptyset$. Now assume the intersection is non-empty, and write $p p^{\prime}=q q^{\prime}=r$ for some $p^{\prime}, q^{\prime}, r$ in $P$. Pick a right LCM for $(p, g)$ and $(q, h)$, which we may assume of the form $(r, j)$ for $j \in G$, and write $(p, g)\left(p^{\prime}, k\right)=(q, h)\left(q^{\prime}, l\right)=(r, j)$ where $k, l$ in $G$ are determined by $j=\vartheta_{q^{\prime}}(h) l=\vartheta_{p^{\prime}}(g) k$. Then $w_{(p, g)}^{*} w_{(q, h)}=w_{\left(p^{\prime}, k\right)} w_{\left(q^{\prime}, l\right)}^{*}$, and this readily implies the Li-relation $e_{I} e_{J}=e_{I \cap J}$ for $I=(p, g)\left(P_{\vartheta} \ltimes G\right)$ and $J=(q, h)\left(P_{\vartheta} \ltimes G\right)$. The remaining relations are easy to see, hence there is a $*$-homomorphism $C^{*}\left(P_{\vartheta} \ltimes G\right) \rightarrow \mathcal{N} \mathcal{T}\left(E_{\alpha}\right)$ which sends a generating isometry $v_{(p, g)}$ 
to $w_{(p, g)}$. Conversely, for $p \in P$ we let

$$
\psi_{p}\left(\delta_{l}\right)=v_{(p, l)} .
$$

We claim that $\psi_{p}: E_{p} \rightarrow C^{*}\left(P_{\vartheta} \ltimes G\right)$ give rise to a Nica covariant representation of $E_{\alpha}$. However, this follows from routine calculations. For example, for $\delta_{g} \in C^{*}(G)$ and $x=$ $\vartheta_{p}(k) l \in E_{p}$ we have

$$
\psi_{p}\left(\delta_{g} \cdot x\right)=\psi_{p}\left(\alpha_{p}\left(\delta_{g}\right) \delta_{\vartheta_{p}(k) l}=\psi_{p}\left(\delta_{\vartheta_{p}(g k) l}\right)\right),
$$

which is $\psi_{e}\left(\delta_{g}\right) \psi_{p}(x)$. As a consequence, there is a $*$-homomorphism $\mathcal{N} \mathcal{T}\left(E_{\alpha}\right) \rightarrow C^{*}\left(P_{\vartheta} \ltimes G\right)$ sending $i_{E_{\alpha}}(x)$ for $x=\alpha_{p}\left(\delta_{k}\right) \delta_{l} \in E_{p}$ to $v_{(1, k)} v_{(p, l)}$. This is an inverse to the homomorphism $C^{*}\left(P_{\vartheta} \ltimes G\right) \rightarrow \mathcal{N} \mathcal{T}\left(E_{\alpha}\right)$ obtained in the first half of the proof, hence the result follows.

Corollary 4.6 (Uniqueness Theorem for $C^{*}\left(P_{\vartheta} \ltimes G\right)$ ). Let $S=P_{\vartheta} \ltimes G$ where $\vartheta$ is a right action of a right LCM semigroup $P$ on a group $G$. Suppose that either $P^{*}=\{e\}$ or that the action of $\left\{\alpha_{h}\right\}_{h \in P^{* o p}}$ on $C^{*}(G)$ is aperiodic. Assume that $\mathcal{K}_{\alpha}$ is amenable. For a Nica covariant representation $(\pi, W)$ of $\left(C^{*}(G), P, \alpha\right)$, cf. Proposition 3.11, we have a canonical surjective homomorphism

$$
C^{*}(S) \mapsto \overline{\operatorname{span}}\left\{W_{p} \pi(a) W_{q}^{*}: a \in \alpha_{p}\left(C^{*}(G)\right) C^{*}(G) \alpha_{q}\left(C^{*}(G)\right), p, q \in P\right\}
$$

which is an isomorphism if and only if for every finite family $q_{1}, \ldots, q_{n}$ in $P \backslash P^{*}$, the representation $a \mapsto \pi(a) \Pi_{i=1}^{n}\left(1-W_{q_{i}} W_{q_{i}}^{*}\right)$ of $C^{*}(G)$ is faithful.

Proof. Combine Proposition 4.5 and Theorem 3.13.

The above result recovers the Laca-Raeburn uniqueness theorem [23] for Nica-Toeplitz algebras in the context of quasi-lattice ordered groups: just take $G=\{e\}$ and $P$ to be any weakly quasi-lattice ordered monoid. It also improves the case (1) in [1, Theorem 4.3], as we do not require (right) cancellativity.

Example 4.7 (Right wreath product). Let $\Gamma$ be a group and $P$ a right LCM semigroup. We form the right wreath product

$$
S:=P \imath \Gamma=P_{\vartheta} \ltimes\left(\prod_{p \in P} \Gamma\right)
$$

with the action given by left shifts $\vartheta_{p}\left(\left(\gamma_{r}\right)_{r \in P}\right):=\left(\gamma_{r p}\right)_{r \in P}$ for $p \in P$ and $\left(\gamma_{r}\right)_{r \in P} \in G=$ $\prod_{p \in P} \Gamma$. Clearly, $\vartheta_{p} \circ \vartheta_{q}=\vartheta_{q p}$ for all $p, q \in P$, as required for a right action. For any $a \in C^{*}(\Gamma)$ and $q \in P$ we let $a \delta_{q}$ be the element of $C^{*}(G)=\prod_{p \in P} C^{*}(\Gamma)$ corresponding to the sequence with $a$ on $q$-th coordinate and zeros elsewhere. The action $\alpha$ by endomorphisms of $C^{*}(G)$ is determined by $\alpha_{p}\left(a \delta_{q}\right)=a \sum_{r p=q} \delta_{r}$ (if $P$ is right cancellative this sum has at most one summand, if this sum is infinite we understand it as a series convergent in weak topopology). In particular, if $h \in P^{*}$, then $\alpha_{h}\left(a \delta_{q}\right)=a \delta_{q h^{-1}}$ and therefore $\left\|\alpha_{h}\left(a \delta_{q}\right) a \delta_{q}\right\|=0$ unless $q h^{-1}=q$ which is equivalent to $h=e$, by left cancellation. Since every non-zero hereditary subalgebra of $C^{*}(G)$ contains a non-zero element of the form $a \delta_{q}$, we conclude that the action $\left\{\alpha_{h}\right\}_{h \in P^{* o p}}$ on $C^{*}(G)$ is always aperiodic. Assuming, for instance, that there is a controlled function from $P$ into an amenable group, we get $\mathcal{K}_{\alpha}$ amenable. Therefore, if $\left\{W_{p}\right\}_{p \in P}$ is a semigroup of isometries on a Hilbert space $H$ satisfying Nica relations (3.7) and $\pi: C^{*}(G) \rightarrow B(H)$ is a nondegenerate representation such that

$$
\left.W_{p}^{*} \pi\left(a \delta_{q}\right)\right) W_{p}=\sum_{r p=q} \pi\left(a \delta_{r}\right), \quad \text { for all } a \in C^{*}(\Gamma), q \in P,
$$

where the sum (if infinite) is convergent in the strong operator topology, then by Corollary 4.6 the surjective homomorphism in (4.2) is an isomorphism if and only if for every $q_{1}, \ldots, q_{n} \in$ 
$P \backslash P^{*}$ and $q \in P$, the representation $C^{*}(\Gamma) \ni a \mapsto \pi\left(a \delta_{q}\right) \Pi_{i=1}^{n}\left(1-W_{q_{i}} W_{q_{i}}^{*}\right)$ is faithful (then the corresponding representation of $C^{*}(G)$ is faithful as well).

4.3. Semigroup $C^{*}$-algebras associated to left semidirect products $G \rtimes_{\theta} P$. Let $(G, P, \theta)$ be an algebraic dynamical system. The authors of [2] associated to $(G, P, \theta)$ a $C^{*}$-algebra $\mathcal{A}[G, P, \theta]$ universal for a unitary representation of $G$ and a Nica covariant isometric representation of $P$ subject to relations that model $\theta$ and the condition of preservation of order. In fact, there is a canonical isomorphism $\mathcal{A}[G, P, \theta] \cong C^{*}\left(G \rtimes_{\theta} P\right)$, see [2, Theorem 4.4]. It was also shown in [2] that $\mathcal{A}[G, P, \theta]$ is naturally isomorphic to the Nica-Toeplitz algebra for a compactly-aligned product system $M$ over $P$ with fibers obtained as completions of $C^{*}(G)$. Specifically, denote by $\delta_{g}$ for $g \in G$ the generating unitaries in $C^{*}(G)$. We have two actions $\alpha: P \rightarrow \operatorname{End}\left(C^{*}(G)\right)$ and $L: P^{o p} \rightarrow \operatorname{Pos}\left(C^{*}(G)\right)$ given by $\alpha_{p}\left(\delta_{g}\right)=\delta_{\theta_{p}(g)}$ and

$$
L_{p}\left(\delta_{g}\right)=\chi_{\theta_{p}(G)}(g) \delta_{\theta_{p}^{-1}(g)}, \quad \text { for } p \in P \text { and } g \in G .
$$

For every $p \in P, L_{p}$ is a transfer operator for $\alpha_{p}$. The product system constructed in [2, Section 7] coincides with the product system $M_{L}$ we defined in subsection 3.3 (for general semigroup actions by transfer operators). By [2, Proposition 7.8], $M_{L}$ is compactly-aligned. Summarizing we get:

Proposition 4.8. Let $(G, P, \theta)$ be an algebraic dynamical system and consider the associated right LCM semigroup $G \rtimes_{\theta} P$. Let $L$ be the action of $P^{o p}$ by transfer operators on $C^{*}(G)$ described in (4.3). Then $\left(C^{*}(G), P, L\right)$ is a $C^{*}$-dynamical system as in subsection 3.3 and there are natural isomorphisms

$$
\mathcal{A}[G, P, \theta] \cong C^{*}\left(G \rtimes_{\theta} P\right) \cong \mathcal{N} \mathcal{T}\left(C^{*}(G), P, L\right) .
$$

Proof. Since $\mathcal{A}[G, P, \theta] \cong \mathcal{N} \mathcal{T}(M)$ by [2, Theorem 7.9], the assertions follow by an application of Proposition 3.19.

Corollary 4.9 (Uniqueness Theorem for $C^{*}\left(G \rtimes_{\theta} P\right)$ ). Let $S=G \rtimes_{\theta} P$ where $(G, P, \theta)$ is an algebraic dynamical system. Suppose that either $P^{*}=\{e\}$ or that the action of $\left\{\alpha_{h}\right\}_{h \in P^{*}}$ on $C^{*}(G)$ is aperiodic. Assume also that $\mathcal{K}_{M_{L}}$ is amenable. Let $(\pi, W)$ be a Nica covariant representation of $\left(C^{*}(G), P, L\right)$, and let $Q_{p}$ be the projection onto the space $\overline{\pi(A) W_{p} H}, p \in P$. We have a surjective homomorphism

$$
C^{*}(S) \mapsto \overline{\operatorname{span}}\left\{\pi(a) W_{p} W_{q}^{*} \pi(b): a \in \alpha_{p}(A) A, b \in \alpha_{q}(A) A, p, q \in P\right\},
$$

which is an isomorphism if for every finite family $q_{1}, \ldots, q_{n}$ in $P \backslash P^{*}$, the representation $a \mapsto \pi(a) \Pi_{i=1}^{n}\left(1-Q_{q_{i}}\right)$ of $C^{*}(G)$ is faithful. If in addition $G / \theta_{p}(G)$ is finite for every $P$, then the latter condition is also necessary for the representation (4.4) to be faithful.

Proof. $C^{*}(S)$ is isomorphic to the Nica-Toeplitz crossed product $\mathcal{N} \mathcal{T}\left(C^{*}(G), P, L\right)$ due to Proposition 4.8. Thus by Proposition 3.19 combined with Lemma 3.12 we may apply Theorem 2.19 to get the sufficiency claim of the isomorphism in (4.4). For the last part, note that the left action of $C^{*}(G)$ on each fiber of $M_{L}$ is by compacts if and only if $G / \theta_{p}(G)$ is finite for every $P$, see [2, Proposition 7.3].

Example 4.10 (Left wreath product). Let $P$ and $\Gamma$ be as in Example 4.7. Form the standard (left) wreath product

$$
S:=\Gamma \imath P=\left(\prod_{p \in P} \Gamma\right) \rtimes_{\theta} P
$$

where $\theta$ acts by right shifts on $G:=\prod_{p \in P} \Gamma$, i.e. $\left(\theta_{p}\left(\left(\gamma_{q}\right)_{q \in P}\right)\right)_{r}=\chi_{p P}(r) \gamma_{p^{-1} r}$ for all $r \in P$. Then $(G, P, \theta)$ is an algebraic dynamical system, cf. [2, Proposition 8.8]. As in 
Example 4.7, for any $a \in C^{*}(\Gamma)$ and $q \in P$ we denote by $a \delta_{q}$ the corresponding element of $C^{*}(G)=\prod_{p \in P} C^{*}(\Gamma)$. For $h \in P^{*}$ we have $\alpha_{h}\left(a \delta_{q}\right)=a \delta_{h q}$ and therefore $\left\|\alpha_{h}\left(a \delta_{q}\right) a \delta_{q}\right\|=0$ unless $h q=q$. Using this, cf. also Example 4.7, we get that

the action $\left\{\alpha_{h}\right\}_{h \in P^{*}}$ on $C^{*}(G)$ is aperiodic $\Longleftrightarrow\left(\forall_{h \in P^{*}} \forall_{q \in P \backslash P^{*}} h q=q \Longrightarrow h=e\right)$.

In particular, $\left\{\alpha_{h}\right\}_{h \in P^{*}}$ is aperiodic when $P^{*}=\{e\}$ or when $P$ is right cancellative. If it is aperiodic we can get a uniqueness criterion for $C^{*}(S)$ using Corollary 4.9. If in addition $G / \theta_{p}(G)$ is finite for every $P$, then the action $L: P^{o p} \rightarrow \operatorname{Pos}\left(C^{*}(G)\right)$ given by (4.3) satisfies assumptions (A1), (A2), (A3) in subsection 3.4. Therefore in this case also Theorem 3.25 applies.

\section{REFERENCES}

[1] N. Brownlowe, N. S. Larsen and N. Stammeier, On $C^{*}$-algebras associated to right LCM semigroups, Trans. Amer. Math. Soc. 369 (2017), no. 1, 31-68.

[2] N. Brownlowe, N. S. Larsen and N. Stammeier, $C^{*}$-algebras of algebraic dynamical systems and right LCM semigroups, to appear in Indiana Univ. Math. J., preprint arXiv:1503.01599v1.

[3] N. Brownlowe, J. Ramagge, D. Robertson and M. F. Whittaker, Zappa-Szép products of semigroups and their $C^{*}$-algebras, J. Funct. Anal. 266 (2014), 3937-3967.

[4] J. Cuntz, Simple $C^{*}$-algebras generated by isometries, Comm. Math. Phys. 57 (1977), 173-185.

[5] S. Doplicher and J. E. Roberts, A new duality theory for compact groups, Invent. Math. 98 (1989), 157-218.

[6] R. Exel, A new look at the crossed-product of a $C^{*}$-algebra by an endomorphism, Ergodic Theory Dynam. Systems, 23 (2003), 1733-1750.

[7] R. Exel, Interactions, J. Funct. Analysis 244 (2007), 26-62.

[8] R. Exel, Partial dynamical systems, Fell bundles and applications, Mathematical Surveys and Monographs, 224. American Mathematical Society, Providence, RI, 2017.

[9] R. Exel and D. Royer, The crossed product by a partial endomorphism, Bull. Braz. Math. Soc. (N.S.) 38 (2007), no. 2, 219-261.

[10] J. Fletcher, A uniqueness theorem for the Nica-Toeplitz algebra of a compactly aligned product system, preprint arXiv:1705.00775.

[11] N. J. Fowler, Discrete product systems of Hilbert bimodules, Pacific J. Math. 204 (2002), 335-375.

[12] N. J. Fowler and I. Raeburn, Discrete product systems and twisted crossed products by semigroups, J. Funct. Anal., 155 (1998), 171-204.

[13] N. J. Fowler and I. Raeburn, The Toeplitz algebra of a Hilbert bimodule, Indiana Univ. Math. J. 48 (1999), $155-181$.

[14] N. J. Fowler and A. Sims Product systems over right-angled Artin semigroups, Trans. Amer. Math. Soc. 354 (2002), 1487-1509.

[15] P. Ghez, R. Lima and J. E. Roberts, $W^{*}$-categories, Pacific. J. Math. 120 (1985), 79-109.

[16] T. Kajiwara, C. Pinzari and Y. Watatani, Hilbert $C^{*}$-bimodules and countably generated Cuntz-Krieger algebras, J. Operator Theory 45 (2001), 3-18.

[17] B. K. Kwaśniewski, $C^{*}$-algebras generalizing both relative Cuntz-Pimsner and Doplicher-Roberts algebras, Trans. Amer. Math. Soc. 365 (2013), 1809-1873.

[18] B. K. Kwaśniewski, Exel's crossed products and crossed products by completely positive maps, Houston J. Math. 43 (2017), 509-567.

[19] B. K. Kwaśniewski and N. S. Larsen, Nica-Toeplitz algebras associated with right tensor $C^{*}$-precategories over right LCM semigroups, submitted, arXiv:1611.08525.

[20] B. K. Kwaśniewski and W. Szymański, Topological aperiodicity for product systems over semigroups of Ore type, J. Funct. Anal. 270 (2016), no. 9, 3453-3504.

[21] B. K. Kwaśniewski and W. Szymański, Pure infiniteness and ideal structure of $C^{*}$-algebras associated to Fell bundles, J. Math. Anal. Appl. 445 (2017), no. 1, 898-943.

[22] B. K. Kwaśniewski and R. Meyer, Aperiodicity, topological freeness and pure outerness: from group actions to Fell bundles Studia Math. 241 (2018), 257-302.

[23] M. Laca and I. Raeburn, Semigroup crossed products and the Toeplitz algebras of nonabelian groups, J. Funct. Anal., 139 (1996), 415-440. 
[24] E. C. Lance, Hilbert $C^{*}$-Modules: A Toolkit for Operator Algebraists, Cambridge University Press, Cambridge, 1995.

[25] N. S. Larsen, Crossed products by abelian semigroups via transfer operators, Ergodic Theory Dynam. Systems 30 (2010), 1147-1164.

[26] M. V. Lawson, Non-commutative Stone duality: inverse semigroups, topological groupoids and $C^{*}$-algebras, Internat. J. Algebra Comput. 22 (2012), no. 6, 1250058, 47 pp.

[27] X. Li, Semigroup $C^{*}$-algebras and amenability of semigroups, J. Funct. Anal. 262 (2010), 4302-4340.

[28] X. Li, Nuclearity of semigroup $C^{*}$-algebras and the connection to amenability, Adv. Math. 244 (2013), $626-662$.

[29] A. Nica, $C^{*}$-algebras generated by isometries and Wiener-Hopf operators, J. Operator Theory, 27 (1992), $17-52$.

[30] M. D. Norling, Inverse semigroup $C^{*}$-algebras associated with left cancellative semigroups, Proc. Edinb. Math. Soc. 57 (2014), no. 2, 533-564.

[31] G. K. Pedersen, $C^{*}$-algebras and their automorphism groups, London Mathematical Society Monographs, vol. 14, Academic Press, London, 1979.

[32] M. V. Pimsner, A class of $C^{*}$-algebras generalizing both Cuntz-Krieger algebras and crossed products by Z, in Free probability theory (Waterloo, ON, 1995), Amer. Math. Soc., Providence, RI, 1997, 189-212.

[33] I. Raeburn and D. P. Williams, Morita equivalence and continuous-trace $C^{*}$-algebras, Math. Surveys and Monographs, vol. 60, Amer. Math. Soc., Providence, RI,

[34] A. Sims and T. Yeend, $C^{*}$-algebras associated to product systems of Hilbert bimodules, J. Operator Theory 64 (2010), 349-376.

[35] Y. Watatani, Index for $\mathrm{C}^{*}$-subalgebras, Mem. Amer. Math. Soc. No. 424, 1990.

Email address: bartoszk@math.uwb.edu.pl

Institute of Mathematics, University of BiaŁystok, ul. K. Ciolkowskiego 1M, 15-245 BiaŁYSTOK, PolAnd

Email address: nadiasl@math.uio.no

Department of Mathematics, University of Oslo, PO Box 1053 Blindern, 0316 Oslo, Norway 